\title{
Revisiting the Charge Transport in Quantum Hall Systems
}

\author{
Tohru Koma \\ Department of Physics, Gakushuin University, Mejiro, Toshima-ku, Tokyo 171-8588, JAPAN \\ e-mail: tohru.koma@gakushuin.ac.jp
}

We reexamine the charge transport induced by a weak electric field in two-dimensional quantum Hall systems in a finite, periodic box at very low temperatures. Our model covers random vector and electrostatic potentials and electron-electron interactions. The resulting linear response coefficients consist of the time-independent term $\sigma_{x y}$ corresponding to the Hall conductance and the linearly time-dependent term $\gamma_{s y} \cdot t$ in the transverse and longitudinal directions $s=x, y$ in a slow switching limit for adiabatically applying the initial electric field. The latter terms $\gamma_{s y} \cdot t$ are due to the acceleration of the electrons by the uniform electric field in the finite and isolated system, and so the time-independent term $\sigma_{y y}$ corresponding to the diagonal conductance which generates dissipation of heat always vanishes. The well known topological argument yields the integral and fractional quantization of the averaged Hall conductance $\overline{\sigma_{x y}}$ over gauge parameters under the assumption on the existence of a spectral gap above the ground state. In addition to this fact, we show that the averaged acceleration coefficients $\overline{\gamma_{s y}}$ are vanishing under the same assumption. In the non-interacting case, the spectral gap between the neighbouring Landau levels persists if the vector and the electrostatic potentials together satisfy a certain condition, and then the Hall conductance $\sigma_{x y}$ without averaging exhibits the exact integral quantization in the infinite volume limit with the vanishing acceleration coefficients. We also estimate their finite size corrections. In the interacting case, the averaged Hall conductance $\overline{\sigma_{x y}}$ for a non-integer filling of the electrons is quantized to a fraction not equal to an integer under the assumption that the potentials satisfy certain conditions in addition to the gap assumption. We also discuss the relation between the fractional quantum Hall effect and Atiyah-Singer index theorem for non-Abelian gauge fields.

KEY WORDS: Charge transport; linear response theory; quantum Hall effect; geometric invariants; non-Abelian gauge fields. 


\section{Contents}

1 Introduction $\quad 3$

$\begin{array}{lll}2 & \text { The model and the main results } & 7\end{array}$

3 Derivation of the linear response coefficients $\quad 15$

4 The system with translation invariance $\quad 20$

5 The linear response coefficients averaged over the gauge parameters 24

5.1 Proof of Theorem $2.2 \ldots \ldots \ldots \ldots \ldots \ldots$

5.2 Fractional quantization and Atiyah-Singer index theorem . . . . . . . . . 30

6 The non-interacting case $\quad 36$

6.1 The single electron Landau Hamiltonian . . . . . . . . . . . . . . . . . 36

6.2 The general electron gases . . . . . . . . . . . . . . . . . . . . . . 38

7 The interacting case $\quad 42$

7.1 Boundedness of the Hall conductance $\sigma_{x y}(\phi) \ldots \ldots$. . . . . . . . . . . 42

7.2 Fractional quantization of the Hall conductance $\sigma_{x y}(\phi) \ldots . . . . . .44$

A Differentiability of the ground-state wavefunctions 46

B Proof of Proposition 6.2

C Proof of Proposition 6.4

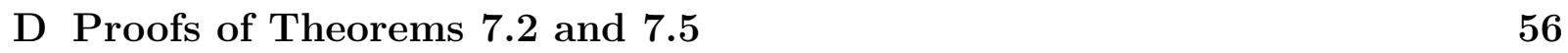

E Proofs of Theorems 7.6, 7.8 and 7.12 59

$\begin{array}{lll}\text { F } & \text { Estimate of the ground state energies } E_{0, \mu}^{(N)}(\phi) & 67\end{array}$ 


\section{Introduction}

The linear response theory $[1,2]$ for charge transport successfully elucidates some aspects of the quantum Hall effect observed experimentally [3, 4] in two-dimensional electron gases in a strong magnetic field. In particular, it was found out that the integral quantization of the Hall conductance is a consequence of a topological nature of the Hall conductance $[5,6]$. However, the derivation of the linear response formulas for conductance from the first principle is still an unsolved problem [7, 8]. Actually it is very hard to take into account the effect of the reservoir explicitly. It is needless to say that there have been many, various arguments, each employing some simplifying feature, so far. For example, the infinite volume formalism without taking the infinite volume limit from a sequence of finite volumes and the adiabatic (slowly varying) switching of the external electric field to avoid accelerating the electrons have been often used instead of coupling the corresponding finite system to a reservoir. ${ }^{1}$ Thus the issue is still left somewhat hanging there although it has been debated again and again so far.

Apart from the problem of the validity of the linear response formulas, the quantized Hall conductance was identified with a topological invariant of a certain fiber bundle [10] by using the resulting linear response formulas as mentioned above. The topological argument for the Hall conductance was first introduced into a quantum Hall system of non-interacting electron gas in a periodic potential $[5,6]$. As a result, it was shown that the Hall conductance is quantized to an integer under the assumption of the existence of a spectral gap above the unique ground state. The integer of the quantization is equal to the filling factor of the Landau levels when the periodic potential is weak. However, one cannot expect the appearance of the conductance plateaus for varying the filling of the electrons because of the absence of disorder. ${ }^{2}$

Soon after these articles, this topological argument ${ }^{3}$ was extended to a quantum Hall system with disorder and with electron-electron interactions [18, 19, 20, 21]. Instead of the crude Hall conductance with fixed gauge parameters, they treated the Hall conductance averaged over gauge parameters, and showed that the averaged Hall conductance is quantized to an integer under the assumption on the existence of a spectral gap above the ground state. The integer of the quantization is equal to the filling factor of the Landau levels in the case of the non-interacting electron gas with weak single-body potentials [11] as well as the above case with a periodic potential. Surprisingly the averaged Hall conductance does not have any finite size correction to the exact integral quantization even though the system has disorder. Clearly one cannot expect that the crude Hall conductance is exactly quantized for any finite system.

In order to explain the fractional quantization [22, 23, 24] of the Hall conductance, this topological argument needs some ad hoc assumptions on the degeneracy of the ground state in addition to the assumption on averaging the Hall conductance [19, 25, 26, 27]. In fact, if the ground state is nondegenerate with an excitation gap, then the averaged Hall conductance always shows integral quantization. The explicit value of the fraction of the

\footnotetext{
${ }^{1}$ For recent attempts to justify the linear response formulas, see refs. [8, 9].

${ }^{2}$ The appearance of the Hall conductance plateaus was discussed with localization estimates in refs. [7, $8,11,12]$. We will discuss this issue in the next paper [13].

${ }^{3}$ See also related articles $[14,15,16,17]$.
} 
quantized Hall conductance is not determined by the topological argument alone even if the dimension of the sector of the degenerate ground state is given. A degenerate ground state with an excitation gap is expected to appear only for a fractional filling $p / q$ of the electrons [28, 29, 30]. Actually, the existence of a spectral gap for a non-integer filling of the electrons implies a degenerate ground state for a certain model [31]. In the real experiments, the fraction of the quantized Hall conductance is observed to equal to the fractional filling $p / q$. Under the assumption on the spectral gap, it was proved without relying on the topological argument that the Hall conductance is proportional to the filling factor of the electrons for certain interacting electron gases [32]. However, the existence of the spectral gap itself has not yet been proved for any interacting electron gas. Besides, the relation between the fractionally quantized Hall conductance and the filling factor of the electrons is still unclear. We should remark that a set of the possible quantized values of the Hall conductance can be derived from a mathematical argument relying on the universality. See ref. [33].

We should also remark another topological approach by Bellissard [8]. For an infinite volume quantum Hall system of a non-interacting electron gas with disorder, the quantized Hall conductance is identified with a Fredholm index of a certain operator that arises in Connes theory of noncommutative geometry [34]. (See also refs. [7, 12, 35, 36].) In comparison to the above topological approach, Bellissard's framework has the advantage that it does not need the assumption on averaging the Hall conductance over gauge parameters. However, it has not yet been extended to interacting quantum Hall electron gases.

In this paper, we study a two-dimensional $N$ electrons system in a uniform magnetic field perpendicular to the two-dimensional plane in which the electrons are confined. For simplicity we assume that the electrons do not have the spin degrees of freedom, although we can treat a similar system with both the spin degrees of freedom and multiple layers in the same way. The explicit form of the Hamiltonian of the system is given by (2.1) in the next section. The model covers a wide class of potentials including a random vector potential, a random electrostatic potential and an electron-electron interaction. In order to measure an induced current as a response to an external electric field, we apply a timedependent vector potential $\mathbf{A}_{\mathrm{ex}}(t)=(0, \alpha(t))$, where the function $\alpha(t)$ of time $t$ is given by (2.14) in the next section. For $t \in[-T, 0]$ with a large positive $T$, the corresponding electric field is adiabatically switched on, and for $t \geq 0$, the electric field becomes $(0, F)$ with the constant strength $F$. We consider the finite, isolated system of an $L_{x} \times L_{y}$ rectangular box, and impose periodic boundary conditions. Thus we will not consider a reservoir, and clearly the system does not exhibit any dissipation of heat. In this sense, we cannot measure the conductance. But the quantum Hall systems in the real experiments show negligibly small dissipation. Correspondingly the system we consider in this paper is expected to show weak acceleration of the electrons. Actually we will show that the acceleration is weak in a certain sense, and the constant Hall current flow is dominant. From these results, if the system is connected with a reservoir, then the acceleration of the electrons is expected to be further suppressed, and the linear response coefficient corresponding to the Hall conductance can be identified with the realistic one. Thus we believe that it is useful in future studies to reexamine the charge transport in such a finite, isolated quantum Hall system.

Let us describe our results. The precise statement of the main results will be given in 
the next section. The linear response coefficients are given by

$$
\sigma_{\mathrm{tot}, s y}=\lim _{F \rightarrow 0} \frac{j_{\text {ind }, s}}{F}
$$

where $j_{\text {ind,s }}$ are the induced current in the transverse direction $s=x$ and the longitudinal direction $s=y$ to the electric field. We obtain the generic forms of the coefficients for time $t \geq 0$ as follows:

$$
\sigma_{\mathrm{tot}, x y}=\sigma_{x y}+\gamma_{x y} \cdot t+\delta \sigma_{x y}
$$

and

$$
\sigma_{\text {tot }, y y}=\gamma_{y y} \cdot t+\delta \sigma_{y y} .
$$

The first term $\sigma_{x y}$ in the right-hand side of (1.2) is constant in time $t$, and corresponds to the Hall conductance. As we mentioned above, the time-independent term $\sigma_{y y}$ corresponding to the diagonal conductance is absent. Instead of that, the linearly time-dependent terms $\gamma_{s y} \cdot t$ appear, i.e., there exist terms corresponding to the acceleration of the electrons by the external electric field. The appearance of the acceleration term $\gamma_{x y} \cdot t$ in the transverse direction is due to the disorder scattering of the electrons accelerated in the longitudinal direction. When the system couples to a reservoir, we can expect that the acceleration terms $\gamma_{s y} \cdot t$ disappear, and instead of them, the diagonal conductance $\sigma_{y y}$ appears . The rest of two terms $\delta \sigma_{s y}$ are the corrections depending on the initial switching process for adiabatically applying the external electric field. We prove that these two terms are negligibly small for slow switching. In the case without a uniform magnetic field, we demonstrate that the velocity of the electrons increases in time for an interacting electron gas with translation invariance in Section 4. More precisely, we have

$$
\sigma_{x y}=0, \quad \gamma_{x y}=0, \quad \text { and } \quad \gamma_{y y}=\frac{n e^{2}}{m_{e}},
$$

where $n$ is the density of the electrons, and $-e$ and $m_{e}$ are, respectively, the charge and the mass of the electron. For an interacting electron gas with translation invariance in a uniform magnetic field [37], we also demonstrate

$$
\sigma_{x y}=-\frac{e^{2}}{h} \nu, \quad \text { and } \quad \gamma_{s y}=0 \quad \text { for } s=x, y,
$$

where $h$ is the Planck constant, and $\nu$ is the filling factor of the Landau levels.

Using the explicit expression of the linear response coefficients, we focus on the unsolved issues about the charge transport of the quantum Hall effect. For this purpose, we assume that there exists an excitation gap above the sector of the (quasi)degenerate ground state. Since our Hall conductance $\sigma_{x y}$ is the same as the standard one, the well-known topological argument yields the fractional quantization of the Hall conductance $[19,25,26,27]$ as

$$
\overline{\sigma_{x y}}=-\frac{e^{2}}{h} \frac{p}{q},
$$

under the assumption on the gap, where $\cdots$ denotes the average over gauge parameters, the integer $p$ is given by the geometrical invariant ${ }^{4}$ called the first Chern number, and the

\footnotetext{
${ }^{4}$ In the following, we will use the term "geometrical" instead of "topological" because we will not consider any deformation for a manifold nor a change of the local coordinate.
} 
integer $q$ is the dimension of the sector of the ground state. Under the same assumption, we prove

$$
\overline{\gamma_{s y}}=0 \quad \text { for } s=x, y \text {. }
$$

Thus the acceleration of the electrons is absent in the sense of the average, and so we can expect that, for fixed gauge parameters, the acceleration of the electrons is weak.

In the non-interacting case, assume an integer filling $\nu=\ell$ of the Landau levels. Then we prove that a spectral gap exists above the unique ground state for certain weak potentials, and that the Hall conductance $\sigma_{x y}$ and the acceleration coefficients $\gamma_{s y}$ satisfy

$$
\left|\sigma_{x y}+\frac{e^{2}}{h} \ell\right| \leq \text { const. } \times \max \left\{L_{x}^{-1}, L_{y}^{-1}\right\},
$$

and

$$
\left|\gamma_{s y}\right| \leq \text { const. } \times \max \left\{L_{x}^{-1}, L_{y}^{-1}\right\} \quad \text { for } s=x, y,
$$

where $L_{x}, L_{y}$ are the system sizes. This Hall conductance $\sigma_{x y}$ is not averaged over the gauge parameters, and the result gives the upper bound for the finite size correction ${ }^{5}$ to the quantized value $-\left(e^{2} / h\right) \ell$. The second inequality for $\gamma_{s y}$ implies weak acceleration of the electrons. In particular, it vanishes in the infinite volume.

In the general case of the interacting electron gas, we cannot remove both of the assumptions on the existence of a spectral gap above the sector of the ground state and on the average over the gauge parameters. About the assumption on the average, for general values of the gauge parameters, we cannot expect an exact fractional quantization of the Hall conductance as (1.6), and the finite-size corrections to the quantized Hall conductance should appear. Besides, the fraction $p / q$ cannot be determined by the geometrical argument alone. But we can get the following result: In addition to the assumption on the existence of the spectral gap, if the potentials satisfy certain technical assumptions, then the fraction $p / q$ must satisfy the bound,

$$
\nu(1-\delta) \leq \frac{p}{q} \leq \nu(1+\delta)
$$

where $\nu$ is the filling factor of the electrons, and $\delta$ is a positive number which is determined by certain norms of the single-body potentials. In order to clarify the meaning of the bound (1.10), consider the situation that the interval $[\nu(1-\delta), \nu(1+\delta)]$ does not include any integer. This situation is indeed realized for a non-integer filling factor $\nu$ and for weak single-body potentials. Then the fraction $p / q$ must be a non-integer, and the degeneracy $q$ of the ground states must be greater than 1. Thus the fractional quantization of the Hall conductance occurs with a degenerate ground state for a non-integer filling.

The present paper is organized as follows: In Section 2, we give the precise definition of the model and describe our main theorems in a mathematical rigorous manner. In Section 3, the linear response coefficients are derived, starting from the basic Schrödinger equation with a time-dependent gauge field which gives a constant electric field for the time $t \geq 0$. We check that the linear response coefficients so obtained are physically reasonable

\footnotetext{
${ }^{5}$ The upper bound for the finite size correction would not be optimal [38]. But the inequality (1.8) gives the mathematical rigorous upper bound!
} 
ones for certain translationally invariant systems in Section 4. Section 5 is devoted to the proofs of the fractional quantization (1.6) of the averaged Hall conductance $\overline{\sigma_{x y}}$ and of the vanishing (1.7) of the averaged acceleration coefficients $\overline{\gamma_{s y}}$ in the most general setting. We also discuss the relation between the fractional quantum Hall effect and Atiyah-Singer index theorem for non-Abelian gauge fields [39, 40, 41, 42]. The non-interacting electron gases with disorder is treated in Section 6. As a result, we obtain the bound (1.8) for the Hall conductance and the bound (1.9) for the acceleration coefficients. In Section 7, the interacting electron gases with disorder are treated, and we prove the bound (1.10) for the fraction of the quantized Hall conductance (1.6). Appendices A-F are devoted to the details of technical calculations and proofs of propositions and theorems.

\section{The model and the main results}

Consider a two-dimensional $N$ electrons system in a uniform magnetic field $(0,0, B)$ perpendicular to the $x-y$ plane in which the electrons are confined. For simplicity we assume that the electrons do not have the spin degrees of freedom, although we can treat a similar system with the spin degrees of freedom or with multiple layers in the same way. The Hamiltonian is given by

$$
H_{0}^{(N)}=\sum_{j=1}^{N}\left\{\frac{1}{2 m_{e}}\left[\mathbf{p}_{j}+e \mathbf{A}\left(\mathbf{r}_{j}\right)+\boldsymbol{\phi}\right]^{2}+W\left(\mathbf{r}_{j}\right)\right\}+\sum_{1 \leq i<j \leq N} W^{(2)}\left(\mathbf{r}_{i}-\mathbf{r}_{j}\right),
$$

where $-e$ and $m_{e}$ are, respectively, the charge of electron and the mass of electron, and $\mathbf{r}_{j}=\left(x_{j}, y_{j}\right)$ is the $j$ th Cartesian coordinate of the $N$ electrons. As usual, we define

$$
p_{x, j}=-i \hbar \frac{\partial}{\partial x_{j}} \quad \text { and } \quad p_{y, j}=-i \hbar \frac{\partial}{\partial y_{j}}
$$

with the Planck constant $\hbar$. The system is defined on a rectangular box,

$$
\mathcal{T}:=\left[-L_{x} / 2, L_{x} / 2\right] \times\left[-L_{y} / 2, L_{y} / 2\right]
$$

with the periodic boundary conditions. The vector potential $\mathbf{A}=\left(A_{x}, A_{y}\right)$ consists of two parts as $\mathbf{A}=\mathbf{A}_{\mathrm{P}}+\mathbf{A}_{0}$, where $\mathbf{A}_{0}(\mathbf{r})=(-B y, 0)$ which gives the uniform magnetic field and the vector potential $\mathbf{A}_{\mathrm{P}}$ satisfies the periodic boundary conditions

$$
\mathbf{A}_{\mathrm{P}}(x, y)=\mathbf{A}_{\mathrm{P}}\left(x+L_{x}, y\right)=\mathbf{A}_{\mathrm{P}}\left(x, y+L_{y}\right)
$$

We have also introduced the gauge parameters $\phi=\left(\phi_{x}, \phi_{y}\right) \in \mathcal{T}_{\mathrm{g}}$, where the space $\mathcal{T}_{\mathrm{g}} \subset \mathbf{R}^{2}$ of the gauge parameters $\phi$ is defined as

$$
\mathcal{T}_{\mathrm{g}}:=\left[0, \Delta \phi_{x}\right] \times\left[0, \Delta \phi_{y}\right] \quad \text { with } \Delta \phi_{s}:=\frac{2 \pi \hbar}{L_{s}}, s=x, y
$$

We call $\mathcal{T}_{\mathrm{g}}$ the gauge torus. As we will see in Section 5 , the Hall conductance $\sigma_{x y}$ of the present system can be expressed in a geometric invariant on the gauge torus $\mathcal{T}_{\mathrm{g}}$. We assume $\mathbf{A}_{\mathrm{P}} \in C^{1}\left(\mathbf{R}^{2}, \mathbf{R}^{2}\right)$, i.e., the components are continuously differentiable on $\mathbf{R}^{2}$. Further we 
assume that the single-body potential $W$ and the electron-electron interaction $W^{(2)}$ satisfy the following conditions: the periodic boundary conditions

$$
W\left(x+L_{x}, y\right)=W\left(x, y+L_{y}\right)=W(x, y)
$$

and

$$
W^{(2)}\left(x+L_{x}, y\right)=W^{(2)}\left(x, y+L_{y}\right)=W^{(2)}(x, y),
$$

and the boundedness ${ }^{6}$

$$
\|W\|_{\infty}<w_{0}<\infty \quad \text { and } \quad\left\|W^{(2)}\right\|_{\infty}<w_{0}^{(2)}<\infty
$$

with the positive constants $w_{0}$ and $w_{0}^{(2)}$ which are independent of the number $N$ of the electrons and of the system sizes $L_{x}, L_{y}$; the interaction $W^{(2)}$ is invariant under the interchange of two electron's coordinates as

$$
W^{(2)}(-x,-y)=W^{(2)}(x, y) \text {. }
$$

For our purpose of accelerating the electrons on the torus $\mathcal{T}$ by the electric field, it is convenient to choose the periodic boundary conditions (2.10) and (2.11) below for the wavefunctions. Then the magnetic flux piercing the torus must be quantized $[31,32]$ so that the Hamiltonian is self-adjoint, or we need additional conditions for the wavefunctions. See ref. [37] for other choices of boundary conditions with a nonquantized flux. In the present paper, we choose the flux quantization condition, $L_{x} L_{y}=2 \pi M \ell_{B}^{2}$, with a sufficiently large positive integer $M$, where $\ell_{B}$ is the so-called magnetic length defined as $\ell_{B}:=\sqrt{\hbar /(e B)}$. The number $M$ is exactly equal to the number of the states in a single Landau level of the single-electron Hamiltonian in the simple uniform magnetic field with no single-body potential and with no electric field. For simplicity we take $M$ even. We define by $\nu=N / M$ the filling factor. We assume $\nu<\nu_{0}$ with a positive constant $\nu_{0}$ which is independent of $L_{x}, L_{y}$ and $N$. In other words, the filling factor $\nu$ converges to a finite constant in an infinite volume limit. The above condition $L_{x} L_{y}=2 \pi M \ell_{B}^{2}$ for the sizes $L_{x}, L_{y}$ is convenient for imposing the following periodic boundary conditions: For an $N$ electrons wavefunction $\Phi^{(N)}$, we impose periodic boundary conditions

$$
t_{j}^{(x)}\left(L_{x}\right) \Phi^{(N)}\left(\mathbf{r}_{1}, \mathbf{r}_{2}, \ldots, \mathbf{r}_{N}\right)=\Phi^{(N)}\left(\mathbf{r}_{1}, \mathbf{r}_{2}, \ldots, \mathbf{r}_{N}\right) \quad \text { for } j=1,2, \ldots, N,
$$

and

$$
t_{j}^{(y)}\left(L_{y}\right) \Phi^{(N)}\left(\mathbf{r}_{1}, \mathbf{r}_{2}, \ldots, \mathbf{r}_{N}\right)=\Phi^{(N)}\left(\mathbf{r}_{1}, \mathbf{r}_{2}, \ldots, \mathbf{r}_{N}\right) \quad \text { for } j=1,2, \ldots, N
$$

where $t^{(x)}(\cdots)$ and $t^{(y)}(\cdots)$ are magnetic translation operators [43] defined as

$$
t^{(x)}\left(x^{\prime}\right) f(x, y)=f\left(x-x^{\prime}, y\right), \quad t^{(y)}\left(y^{\prime}\right) f(x, y)=\exp \left[i y^{\prime} x / \ell_{B}^{2}\right] f\left(x, y-y^{\prime}\right)
$$

\footnotetext{
${ }^{6}$ Let $f$ be a complex-valued function on $\mathbf{R}^{2}$, and let $|f(x, y)| \leq \mathcal{C}$ for some $\mathcal{C}$ except for a subset of Lebesgue measure zero in $\mathbf{R}^{2}$. Then the norm $\|f\|_{\infty}$ is given by the smallest such $\mathcal{C}$. If $f$ is a continuous function, then $\|f\|_{\infty}=\max |f(x, y)|$.
} 
for a function $f$ on $\mathbf{R}^{2}$, and a subscript $j$ of an operator indicates that the operator acts on only the $j$-th coordinate of the function. ${ }^{7}$

In order to get the expression of the current induced by an external electric field, we introduce a time-dependent vector potential [44] into the Hamiltonian (2.1) as

$$
\begin{aligned}
H^{(N)}(t) & =\sum_{j=1}^{N}\left\{\frac{1}{2 m_{e}}\left[p_{x, j}+e A_{x}\left(\mathbf{r}_{j}\right)+\phi_{x}\right]^{2}+\frac{1}{2 m_{e}}\left[p_{y, j}+e A_{y}\left(\mathbf{r}_{j}\right)+\phi_{y}+e \alpha(t)\right]^{2}\right\} \\
& +\sum_{j=1}^{N} W\left(\mathbf{r}_{j}\right)+\sum_{1 \leq i<j \leq N} W^{(2)}\left(\mathbf{r}_{i}-\mathbf{r}_{j}\right)
\end{aligned}
$$

where the additional vector potential is given by $\mathbf{A}_{\mathrm{ex}}(t)=(0, \alpha(t))$ with

$$
\alpha(t)=-F t \times \begin{cases}e^{\eta t}, & t \leq 0 \\ 1, & t>0\end{cases}
$$

with a small positive parameter $\eta$. The corresponding electric field is oriented along the $y$ direction with the constant strength $F$ for all $t \geq 0$. Namely we apply the electric field adiabatically from the initial time $t=t_{0}=-T$ with a large $T$, and observe the currents of the system for the time $t \geq 0$. Therefore we will consider those quantities for the time $t \geq 0$ only in the following.

Throughout the present paper, we will consider the following two situations: (i) The ground state of the Hamiltonian $H_{0}^{(N)}$ of $(2.1)$ is exactly $q$-fold degenerate, $(q=1,2, \ldots)$, with a small excitation energy gap which tends to zero in the infinite volume limit. (ii) The ground state is $q$-fold quasidegenerate with a uniform excitation energy gap which persists in the infinite volume limit in the sense of Assumption 2.1 below. In both of two situations, we take the initial state of the system at the time $t=t_{0}=-T$ as

$$
\omega_{0}(\cdots):=\frac{1}{q} \sum_{\mu=1}^{q}\left\langle\Phi_{0, \mu}^{(N)}(\phi),(\cdots) \Phi_{0, \mu}^{(N)}(\phi)\right\rangle,
$$

where $\Phi_{0, \mu}^{(N)}(\phi)$ are the $q$ eigenvectors of the ground state of the Hamiltonian $H_{0}^{(N)}$ of $(2.1)$. Namely we assume that the system is at a low temperature such that the corresponding inverse temperature $\beta$ satisfies the condition $\Delta \mathcal{E} \ll \beta^{-1} \ll \Delta E$, where $\Delta E$ is the excitation energy gap and $\Delta \mathcal{E}=\max _{\mu, \mu^{\prime}}\left|E_{0, \mu}^{(N)}(\phi)-E_{0, \mu^{\prime}}^{(N)}(\phi)\right|$ with the energy eigenvalue $E_{0, \mu}^{(N)}(\phi)$ of the ground state eigenvector $\Phi_{0, \mu}^{(N)}(\phi)$. In the corresponding realistic situation, the transition between the degenerate ground states frequently occurs with finite probabilities owing to an external thermal perturbation. In consequence, all of the ground states are equally mixed as in the assumption of the initial state (2.15). But, when a symmetry breaking occurs at zero temperature, those transition probabilities become negligibly small in a large volume. In that situation, the assumption of the initial state (2.15) may be physically unnatural. Instead of the mixed state (2.15), we might have to take one of the symmetry breaking pure ground states as an initial state. But we can expect that all of the pure ground states give the same current because the broken symmetry is the translational symmetry $[31,32]$. Here we stress that we cannot justify this argument. To

\footnotetext{
${ }^{7}$ Throughout the present paper, we use this convention.
} 
summarize, in both of the cases, we can expect that the assumption of the initial state (2.15) leads to the realistic, correct current for the present quantum Hall system.

In general, it is believed that the existence of an energy gap above the ground state for an integral or a fractional filling of the electrons is essential to both integral and fractional quantization of the Hall conductance. In addition, the degeneracy [19, 27] of the ground state is essential to the fractional quantization because the unique ground state with an energy gap always yields an integral quantization of the conductance by using the well-known topological argument [19, 20]. However, as Tao and Haldane [26] pointed out, one cannot expect the exact degeneracy of the ground state because the randomness of the potential(s) always lifts the degeneracy for a finite system. Therefore we require the following assumption on the quasidegeneracy of the ground state with the excitation energy gap in the quantum Hall case:

Assumption 2.1 For any $\phi \in \mathcal{T}_{\mathrm{g}}$, the ground state of the Hamiltonian $H_{0}^{(N)}$ of (2.1) is $q$-fold degenerate in the sense that

$$
\max _{\mu, \mu^{\prime} \in\{1,2, \ldots, q\}}\left|E_{0, \mu}^{(N)}(\phi)-E_{0, \mu^{\prime}}^{(N)}(\phi)\right| \rightarrow 0 \text { as } L_{x}, L_{y} \rightarrow \infty
$$

where $E_{0, \mu}^{(N)}(\phi), \mu=1,2, \ldots, q$, are the energy eigenvalues of the ground state. Besides, there exists a uniform energy gap $\Delta E$ above the degenerate ground state in the sense that

$$
\inf _{\phi \in \mathcal{T}_{\mathrm{g}}} E_{1}^{(N)}(\phi)>\Delta E+\sup _{\boldsymbol{\phi} \in \mathcal{T}_{\mathrm{g}}} \max _{\mu \in\{1,2, \ldots, q\}} E_{0, \mu}^{(N)}(\phi)
$$

where $E_{1}^{(N)}(\phi)$ is the energy of the first excited state, and $\Delta E$ is a positive constant which is independent of the number $N$ of the electrons and of the system sizes $L_{x}, L_{y}$.

This assumption is justified for the non-interacting case with the potentials $\mathbf{A}_{\mathrm{P}}$ and $W$ satisfying the condition (2.36) in Theorem 2.3 below. Unfortunately, for the interacting case, we cannot justify the assumption of the gap. We call the subspace of the (quasi)degenerate ground state the sector of the ground state. We remark that the dimension $q$ of the sector of the ground state may depend on the system sizes $L_{x}, L_{y}$ and on the number $N$ of the electrons.

The state of the system at the time $t \geq t_{0}$ is given by

$$
\omega(\cdots ; t):=\omega_{0}\left(\left[U^{(N)}\left(t, t_{0}\right)\right]^{\dagger}(\cdots) U^{(N)}\left(t, t_{0}\right)\right) .
$$

Here $U^{(N)}\left(t, t_{0}\right)$ is the time evolution operator for the Schrödinger equation,

$$
i \hbar \frac{\partial}{\partial t} \Psi^{(N)}(t)=H^{(N)}(t) \Psi^{(N)}(t)
$$

with the time dependent Hamiltonian $H^{(N)}(t)$ of (2.13). Namely the solution of the equation is written as

$$
\Psi^{(N)}(t)=U^{(N)}\left(t, t_{0}\right) \Psi^{(N)}\left(t_{0}\right)
$$

in terms of the operator $U^{(N)}\left(t, t_{0}\right)$ with the initial vector $\Psi^{(N)}\left(t_{0}\right)$. 
Now we define the total velocity operator as

$$
v_{\text {tot }, i}(t):= \begin{cases}\sum_{j=1}^{N} \frac{1}{m_{e}}\left[p_{x, j}+e A_{x}\left(\mathbf{r}_{j}\right)+\phi_{x}\right], & i=x \\ \sum_{j=1}^{N} \frac{1}{m_{e}}\left[p_{y, j}+e A_{y}\left(\mathbf{r}_{j}\right)+\phi_{y}+e \alpha(t)\right], & i=y\end{cases}
$$

Then the total current density is given by

$$
\mathbf{j}_{\mathrm{tot}}(t):=-\frac{e}{L_{x} L_{y}} \omega\left(\mathbf{v}_{\mathrm{tot}}(t) ; t\right),
$$

where $\mathbf{v}_{\text {tot }}(t)=\left(v_{\text {tot }, x}(t), v_{\text {tot }, y}(t)\right)$. This total current density consists of the initial current density $\mathbf{j}_{0}$ and the induced current density $\mathbf{j}_{\text {ind }}(t)$ due to the external electric field as

$$
\mathbf{j}_{\text {tot }}(t)=\mathbf{j}_{0}+\mathbf{j}_{\text {ind }}(t)
$$

where

$$
\mathbf{j}_{0}=-\frac{e}{L_{x} L_{y}} \omega_{0}\left(\mathbf{v}_{\text {tot }}^{(0)}\right)
$$

with the velocity operator,

$$
\mathbf{v}_{\text {tot }}^{(0)}=\sum_{j=1}^{N} \frac{1}{m_{e}}\left[\mathbf{p}_{j}+e \mathbf{A}\left(\mathbf{r}_{j}\right)+\boldsymbol{\phi}\right],
$$

without the vector potential $\alpha(t)$ giving the external electric field. The initial current density $\mathbf{j}_{0}$ is not necessarily vanishing because the persistent current may exist owing to the presence of the vector potentials. The linear response coefficients are given by

$$
\sigma_{\text {tot }, s y}(t ; \phi, \eta, T):=\lim _{F \rightarrow 0} \frac{j_{\text {ind }, s}(t)}{F}
$$

in the $s=x, y$ directions, where we have written $\mathbf{j}_{\text {ind }}(t)=\left(j_{\text {ind }, x}(t), j_{\text {ind }, y}(t)\right)$. As we will show in the next Section 3, these coefficients for the time $t \geq 0$ have the expressions,

$$
\sigma_{\text {tot }, x y}(t ; \phi, \eta, T)=\sigma_{x y}(\phi)+\gamma_{x y}(\phi) \cdot t+\delta \sigma_{x y}(t ; \phi, \eta, T),
$$

and

$$
\sigma_{\text {tot }, y y}(t ; \phi, \eta, T)=\gamma_{y y}(\phi) \cdot t+\delta \sigma_{y y}(t ; \phi, \eta, T),
$$

where $\sigma_{x y}(\phi), \gamma_{x y}(\phi)$ and $\gamma_{y y}(\phi)$ are all independent of the time $t$. The rest of the two terms $\delta \sigma_{s y}(t ; \phi, \eta, T)$ for $s=x, y$ are due to the initial switching process for adiabatically applying the electric field in the time $-T \leq t \leq 0$, and so the two terms are negligibly small for the slow switching condition $\eta T \gg 1$ and $\eta \ll \Delta E / \hbar$. In particular, they vanish in the slow switching limit $\eta T \rightarrow \infty$ and $\eta \rightarrow 0$. See the bound (4.4) and the equality (4.25) in Section 4 for certain translationally invariant systems, the bound (6.27) in Section 6 for the non-interacting electron gas and the bound (7.5) in Section 7 for the interacting electron gas. The term $\sigma_{x y}(\phi)$ corresponds to the Hall conductance. For simplicity, we will call 
$\sigma_{x y}(\phi)$ the Hall conductance. As mentioned in Introduction, the time-independent term $\sigma_{y y}(\phi)$ corresponding to the diagonal conductance does not appear [7, 9, 45], and instead of that, there appear linearly time-dependent terms, $\gamma_{s y}(\phi) \cdot t$. In particular, if the system is translationally invariant in both $x$ and $y$ directions with no uniform magnetic field, then the total velocity of the electrons is proportional to the time $t$ owing to the uniform electric field. (See Section 4.) Therefore we will call $\gamma_{s y}(\phi)$ the acceleration coefficient.

On the other hand, when a finite energy gap appears above the sector of the ground state as in a band insulator or in the quantum Hall case, we can expect that both of the acceleration coefficients $\gamma_{s y}(\phi)$ vanish in the infinite volume limit. Actually this statement holds for the non-interacting case as we will see in Theorem 2.3 below. However, we could not obtain a similar theorem for the interacting case except for a trivial case without disorder in Section 4. Of course, we cannot expect that the acceleration coefficients $\gamma_{s y}(\phi)$ are exactly vanishing for a finite volume in a generic situation with disorder because there may exist non-vanishing current due to the scattering of the electrons by the disorder.

If the Hall conductance $\sigma_{x y}(\phi)$ alone is needed, then it is enough to measure the linear response coefficients at the time $t=0$ because the effect of the Lorentz force alone persists at $t=0$ without any acceleration of the electrons by the potentials of the system.

We define the averaged Hall conductance and the averaged acceleration coefficients over the gauge parameters $\phi$ on the gauge torus $\mathcal{T}_{\mathrm{g}}$ as

$$
\overline{\sigma_{x y}(\boldsymbol{\phi})}=\frac{1}{\Delta \phi_{x} \Delta \phi_{y}} \int_{\mathcal{T}_{\mathrm{g}}} d \phi_{x} d \phi_{y} \sigma_{x y}(\boldsymbol{\phi}),
$$

and

$$
\overline{\gamma_{s y}(\phi)}=\frac{1}{\Delta \phi_{x} \Delta \phi_{y}} \int_{\mathcal{T}_{\mathrm{g}}} d \phi_{x} d \phi_{y} \gamma_{s y}(\phi) \text { for } s=x, y .
$$

Under Assumption 2.1, the averaged Hall conductance $\overline{\sigma_{x y}(\phi)}$ can be written as

$$
\overline{\sigma_{x y}(\phi)}=\frac{e^{2}}{h} \frac{\mathcal{I}}{q}
$$

in terms of the geometric invariant $[5,6,8,19,20,27]$,

$$
\mathcal{I}=\frac{1}{2 \pi i} \int_{\mathcal{T}_{\mathrm{g}}} d \phi_{x} d \phi_{y} \operatorname{tr} \mathcal{F}(\boldsymbol{\phi}),
$$

with the curvature $\mathcal{F}(\phi)$ given by

$$
\mathcal{F}(\phi)=\frac{\partial}{\partial \phi_{x}} \mathcal{A}_{y}(\phi)-\frac{\partial}{\partial \phi_{y}} \mathcal{A}_{x}(\phi)+\left[\mathcal{A}_{x}(\phi), \mathcal{A}_{y}(\phi)\right],
$$

where tr stands for the trace of the matrix, and $\mathcal{A}_{s}(\phi)$ for $s=x, y$ are the connections on the gauge torus $\mathcal{T}_{\text {g }}$, i.e., each $\mathcal{A}_{s}(\phi)$ takes a $q \times q$ matrix value in the Lie algebra of the unitary group $U(q)$ of $q \times q$ matrices [39]. As we will see in Section 5, the connections $\mathcal{A}_{s}(\phi)$ are written in terms of the ground state wavefunctions. In the language of the nonAbelian gauge theory [42], the curvature $\mathcal{F}(\phi)$ corresponds to the field strength tensor or the "electromagnetic" field, and the connections $\mathcal{A}_{s}(\phi)$ correspond to the gauge fields on 
the torus $\mathcal{T}_{\mathrm{g}}$. Thus the averaged Hall conductance can be written in terms of the geometric invariant for the non-Abelian gauge fields on the torus. Since the geometric invariant $\mathcal{I}$ called the first Chern number takes an integer value, the averaged Hall conductance $\overline{\sigma_{x y}(\phi)}$ is integrally or fractionally quantized.

On the other hand, we can prove that the averaged acceleration coefficients $\overline{\gamma_{s y}(\phi)}$ are exactly vanishing. We summarize the well-known result of the averaged Hall conductance $\overline{\sigma_{x y}(\phi)}$ and our result about the averaged acceleration coefficients $\overline{\gamma_{s y}(\phi)}$ as the following theorem in the most general setting for the present model:

Theorem 2.2 Suppose that, for a finite volume and a filling factor $\nu$ of the Landau levels, there exists a uniform gap above the sector of the ground state in the sense of Assumption 2.1. Then there exists an integer $p$ such that the averaged Hall conductance for the finite volume is quantized as

$$
\overline{\sigma_{x y}(\phi)}=-\frac{e^{2}}{h} \frac{p}{q}
$$

with the dimension $q$ of the sector of the ground state, and the averaged acceleration coefficients are vanishing as

$$
\overline{\gamma_{s y}(\phi)}=0 \quad \text { for both } s=x, y \text { directions. }
$$

The proof will be given in Section 5. In such a general setting, we cannot determine the fraction $p / q$, which is expected to be equal to the filling factor $\nu$ of the Landau levels [32]. We also remark that, for general fixed values of the gauge parameters $\phi \in \mathcal{T}_{\text {g }}$, we cannot expect the same exact results about the fractional quantization of the Hall conductance and the vanishing acceleration coefficients without any finite size correction.

For the quantum Hall case without the electron-electron interaction, we can obtain much stronger results as follows:

Theorem 2.3 Assume $W^{(2)}=0$, i.e., no electron-electron interaction, and assume an integer filling factor $\nu=\ell$ of the Landau levels with $\ell \in \mathbf{N}$. Further we assume that the vector potential $\mathbf{A}_{\mathrm{P}}$ and the electrostatic potential $W$ satisfy the condition,

$$
\hbar \omega_{c}>\sqrt{\frac{2 \hbar \omega_{c}}{m_{e}}} e\left\|\left|\mathbf{A}_{\mathrm{P}}\right|\right\|_{\infty}[\sqrt{\ell+1 / 2}+\sqrt{\ell-1 / 2}]+\frac{e^{2}}{2 m_{e}}\left(\left\|\left|\mathbf{A}_{\mathrm{P}}\right|\right\|_{\infty}\right)^{2}+\left\|W^{+}\right\|_{\infty}+\left\|W^{-}\right\|_{\infty},
$$

where $\omega_{c}$ is the cyclotron frequency given by $\omega_{c}=e B / m_{e},\left|\mathbf{A}_{\mathrm{P}}\right|=\sqrt{\mathbf{A}_{\mathrm{P}} \cdot \mathbf{A}_{\mathrm{P}}}$, and $W^{ \pm}=$ $\max \{ \pm W, 0\}$. Then there exists a uniform gap above the unique ground state in the sense of Assumption 2.1, and the following bounds are valid:

$$
\left|\sigma_{x y}\left(\phi_{0}\right)+\frac{e^{2}}{h} \ell\right| \leq \mathcal{C} \max \left\{L_{x}^{-1}, L_{y}^{-1}\right\}
$$

and

$$
\left|\gamma_{s y}\left(\phi_{0}\right)\right| \leq \mathcal{C}^{\prime} \max \left\{L_{x}^{-1}, L_{y}^{-1}\right\}
$$

for any gauge parameters $\phi_{0} \in \mathcal{T}_{\mathrm{g}}$, and for $s=x, y$, where the positive constants $\mathcal{C}$ and $\mathcal{C}^{\prime}$ are independent of the number $N$ of the electrons and of the system sizes $L_{x}, L_{y}$. 
The proof will be given in Section 6. Thus the Hall conductance shows the integral quantization with the finite size correction, and the acceleration coefficients are vanishing in the infinite volume limit. However, the bounds for the finite size corrections would not be optimal [38] in comparison with the precision of the quantization and the weakness of the dissipation of heat in realistic quantum Hall systems. Perhaps, if possible, we should take account of the effect of the self-averaging about the disorder in the realistic systems.

In order to explain the appearance of the Hall conductance plateaus, the gap condition in Theorem 2.3 must be replaced with localization estimates. Namely we must show that the quantized Hall conductance does not change when varying the Fermi level within the localization regime. For infinite volume systems, the Hall conductance formula by Bellissard shows the plateaus [7, 8, 12]. Kunz [11] also discussed the plateaus in an infinite volume system by combining the topological argument with certain assumptions on localization. This issue for finite volume systems will be discussed in ref. [13].

For the interacting case, we could not estimate the corresponding finite size corrections. But we obtain the following theorem about the relation between the fraction $p / q$ of the quantization and the filling factor $\nu$ of the electrons:

Theorem 2.4 Assume $\mathbf{A}_{\mathrm{P}}=0$, and that the electrostatic potential $W$ and the electronelectron interaction $W^{(2)}$ satisfy $W \in C^{3}\left(\mathbf{R}^{2}\right)$ and $W^{(2)} \in C^{1}\left(\mathbf{R}^{2}\right)$, respectively. ${ }^{8}$ Further we assume that, for a finite volume and a filling factor $\nu$ of the Landau levels, there exists a uniform gap $\Delta E$ above the sector of the ground state with the degeneracy $q$ in the sense of Assumption 2.1. Then the fraction $p / q$ of the averaged Hall conductance $\overline{\sigma_{x y}(\phi)}$ of (2.34) satisfies

$$
\nu(1-\delta) \leq \frac{p}{q} \leq \nu(1+\delta)
$$

where the positive number $\delta$ is given by

$$
\delta=2 \ell_{B}^{4} \frac{\hbar \omega_{c}}{(\Delta E)^{3}} \max _{\substack{m, n \geq 0 ; \\ m+n=2}}\left\|\frac{\partial^{m+n} W}{\partial x^{m} \partial y^{n}}\right\|_{\infty}^{2} .
$$

The proof will be given in Section 7.2. As we mentioned in Introduction, if the interval $[\nu(1-\delta), \nu(1+\delta)]$ does not include any integer, then the number $p / q$ must be equal to purely a fractional number, i.e., a non-integer. In addition, the dimension $q$ of the sector of the ground state must be greater than 1 . In the weak potential limit $\delta \rightarrow 0$, we obtain the desired result,

$$
\overline{\sigma_{x y}(\phi)}=-\frac{e^{2}}{h} \nu, \quad \text { with } \nu=p / q
$$

from the result (2.34) with the inequality (2.39). Therefore one can expect the fractional quantization of the Hall conductance [31, 32] when a spectral gap appears above the sector of the ground state for the fractional filling factor $\nu=p / q$. But the Hall conductance would vanish in the exceptional case with a very strong periodic potential. ${ }^{9}$

\footnotetext{
${ }^{8}$ The set $C^{k}(S)$ denotes $k$ times continuously differentiable functions on the set $S$.

${ }^{9}$ When a spectral gap exists owing to a strong periodic potential irrespective of the uniform magnetic field, the Hall conductance must vanish in the weak limit of the magnetic field. In such a situation, the integer $p$ in the fractionally quantized Hall conductance must be equal to zero because the Hall conductance is a continuous function of the uniform magnetic field. Thus the Hall conductance vanishes owing to the strong periodic potential [46].
} 
In the case with $\mathbf{A}_{\mathrm{P}} \neq 0$, we can also obtain very similar results to Theorem 2.4. But we need stronger assumptions on the potentials $\mathbf{A}_{\mathrm{P}}, W$ and $W^{(2)}$. See Section 7.2 for the details.

\section{Derivation of the linear response coefficients}

In this section, we derive the expressions for the linear response coefficients by using the perturbation theory [47] in a mathematically rigorous manner. We denote by $\Phi_{0, \mu}^{(N)}(\phi)$ the ground state eigenvectors of the Hamiltonian $H_{0}^{(N)}$ of $(2.1)$ with the eigenvalue $E_{0, \mu}^{(N)}(\phi)$, and denote by $\Phi_{n, \mu}^{(N)}(\phi)$ with $n \geq 1$ the eigenvectors of the excited states with the energy eigenvalue $E_{n}^{(N)}(\phi)$ and with the subscript $\mu$ for the degeneracy. In the following, we will often use the abbreviations, $\Phi_{n, \mu}^{(N)}, E_{0, \mu}^{(N)}, E_{n}^{(N)}$, by dropping the $\phi$ dependence if there is no confusion.

To begin with, we rewrite the Hamiltonian $H^{(N)}(t)$ of $(2.13)$ as

$$
H^{(N)}(t)=H_{0}^{(N)}+\Delta H_{0}^{(N)}(t)+H_{\mathrm{per}}^{(N)}(t)
$$

with the diagonal part,

$$
\Delta H_{0}^{(N)}(t)=\sum_{n} Q\left(E_{n}^{(N)}\right) H_{\min }^{(N)}(t) Q\left(E_{n}^{(N)}\right)+N \frac{e^{2}}{2 m_{e}}[\alpha(t)]^{2},
$$

of the perturbation and the off-diagonal part,

$$
H_{\mathrm{per}}^{(N)}(t)=H_{\min }^{(N)}(t)-\sum_{n} Q\left(E_{n}^{(N)}\right) H_{\min }^{(N)}(t) Q\left(E_{n}^{(N)}\right),
$$

where $H_{\mathrm{min}}^{(N)}(t)$ is the minimal coupling with the external electric field, i.e.,

$$
H_{\min }^{(N)}(t)=\frac{e}{m_{e}} \sum_{j=1}^{N} \alpha(t)\left[p_{y, j}+e A_{y}\left(\mathbf{r}_{j}\right)\right]
$$

and $Q\left(E_{0}^{(N)}\right)$ is the projection operator onto the subspace spanned by the ground state eigenvectors $\Phi_{0, \mu}^{(N)}$ of the unperturbed Hamiltonian $H_{0}^{(N)}$, and $Q\left(E_{n}^{(N)}\right)$ for $n \geq 1$ is the projection operator onto the eigenspace spanned by the excited state eigenvector(s) of the Hamiltonian $H_{0}^{(N)}$ with the eigenvalue $E_{n}^{(N)}$.

Consider the time-dependent Schrödinger equation

$$
i \hbar \frac{\partial}{\partial t} \Psi^{(N)}(t)=H^{(N)}(t) \Psi^{(N)}(t)
$$

with the Hamiltonian $H^{(N)}(t)$ of $(2.13)$. The solution $\Psi^{(N)}(t)$ can be written as $\Psi^{(N)}(t)=$ $U^{(N)}\left(t, t_{0}\right) \Psi^{(N)}\left(t_{0}\right)$ by using the time evolution operator $U^{(N)}(t, s)$, with an initial vector $\Psi^{(N)}\left(t_{0}\right)$ at the initial time $t=t_{0}$. We denote by $U_{0}^{(N)}(t, s)$ the time evolution operator for the Hamiltonian $H_{0}^{(N)}+\Delta H_{0}^{(N)}(t)$. 
Let $\Psi^{(N)}(t)$ be a solution of the Schrödinger equation (3.5). Note that

$$
\begin{aligned}
& \frac{\partial}{\partial s} U_{0}^{(N)}(t, s) \Psi^{(N)}(s) \\
= & \frac{i}{\hbar} U_{0}^{(N)}(t, s)\left\{H_{0}^{(N)}+\Delta H_{0}^{(N)}(s)\right\} \Psi^{(N)}(s)+U_{0}^{(N)}(t, s) \frac{\partial}{\partial s} \Psi^{(N)}(s) \\
= & \frac{i}{\hbar} U_{0}^{(N)}(t, s)\left\{H_{0}^{(N)}+\Delta H_{0}^{(N)}(s)-H^{(N)}(s)\right\} \Psi^{(N)}(s) \\
= & -\frac{i}{\hbar} U_{0}^{(N)}(t, s) H_{\text {per }}^{(N)}(s) \Psi^{(N)}(s) .
\end{aligned}
$$

Integrating this on the time $s$ from $t_{0}$ to $t$, one obtains

$$
\Psi^{(N)}(t)-U_{0}^{(N)}\left(t, t_{0}\right) \Psi^{(N)}\left(t_{0}\right)=-\frac{i}{\hbar} \int_{t_{0}}^{t} d s U_{0}^{(N)}(t, s) H_{\mathrm{per}}^{(N)}(s) \Psi^{(N)}(s) .
$$

From the definition of $U^{(N)}(t, s)$, this can be rewritten as

$$
\left[U^{(N)}\left(t, t_{0}\right)-U_{0}^{(N)}\left(t, t_{0}\right)\right] \Psi^{(N)}\left(t_{0}\right)=-\frac{i}{\hbar} \int_{t_{0}}^{t} d s U_{0}^{(N)}(t, s) H_{\mathrm{per}}^{(N)}(s) \Psi^{(N)}(s) .
$$

Since both $U^{(N)}(t, s)$ and $U_{0}^{(N)}(t, s)$ are bounded, one has the following lemma [47]:

Lemma 3.1 In the strong sense, $U^{(N)}(t, s) \rightarrow U_{0}^{(N)}(t, s)$ as $F \rightarrow 0$, uniformly in $t, s$ in any finite interval.

In the equation (3.7), we take the initial state at $t_{0}=-T$ as

$$
\Psi^{(N)}\left(t_{0}=-T\right)=U_{0}^{(N)}(-T, 0) \Phi^{(N)}
$$

with a vector $\Phi^{(N)}$ in the domain of the Hamiltonian $H_{0}^{(N)}$ of $(2.1)$. Then the equation (3.7) becomes

$$
\Psi^{(N)}(t)=U_{0}^{(N)}(t, 0) \Phi^{(N)}-\frac{i}{\hbar} \int_{-T}^{t} d s U_{0}^{(N)}(t, s) H_{\mathrm{per}}^{(N)}(s) \Psi^{(N)}(s) .
$$

The following theorem can be obtained in the same way as in Theorem 2.19 in Sec. 2 in Chap. IX of ref. [47]:

Theorem 3.2 Let $\Phi^{(N)}$ be a vector in the domain of the Hamiltonian $H_{0}^{(N)}$ of (2.1). Then

$$
\Psi^{(N)}(t)=U_{0}^{(N)}(t, 0) \Phi^{(N)}-\frac{i}{\hbar} \int_{-T}^{t} d s U_{0}^{(N)}(t, s) H_{\mathrm{per}}^{(N)}(s) U_{0}^{(N)}(s, 0) \Phi^{(N)}+o(F),
$$

where $o(F)$ denotes a vector $\Psi_{\mathrm{R}}$ with the norm $\left\|\Psi_{\mathrm{R}}\right\|$ satisfying $\left\|\Psi_{\mathrm{R}}\right\| / F \rightarrow 0$ as $F \rightarrow 0$.

Proof: Since

$$
\Psi^{(N)}(s)=U^{(N)}(s,-T) \Psi^{(N)}(-T)
$$


and

$$
U_{0}^{(N)}(s, 0) \Phi^{(N)}=U_{0}^{(N)}(s,-T) U_{0}^{(N)}(-T, 0) \Phi^{(N)}=U_{0}^{(N)}(s,-T) \Psi^{(N)}(-T),
$$

it is sufficient to show that

$$
\left\|H_{\mathrm{per}}^{(N)}(s)\left[U^{(N)}(s,-T)-U_{0}^{(N)}(s,-T)\right] \Psi^{(N)}(-T)\right\|=o(F) .
$$

Note that

$$
H_{\mathrm{per}}^{(N)}(s) U^{(N)}(s,-T) \Psi^{(N)}(-T)=H_{\mathrm{per}}^{(N)}(s)[S(s)]^{-1} \tilde{U}^{(N)}(s,-T) S(-T) \Psi^{(N)}(-T)
$$

and

$$
H_{\mathrm{per}}^{(N)}(s) U_{0}^{(N)}(s,-T) \Psi^{(N)}(-T)=H_{\mathrm{per}}^{(N)}(s)\left[\tilde{S}_{0}(s)\right]^{-1} \tilde{U}_{0}^{(N)}(s,-T) \tilde{S}_{0}(-T) \Psi^{(N)}(-T),
$$

where

$$
\begin{gathered}
\tilde{U}(t, s)=S(t) U(t, s)[S(s)]^{-1}, \\
S(t)=\frac{i}{\hbar} H^{(N)}(t)+\lambda_{0}, \\
\tilde{U}_{0}(t, s)=\tilde{S}_{0}(t) U_{0}(t, s)\left[\tilde{S}_{0}(s)\right]^{-1},
\end{gathered}
$$

and

$$
\tilde{S}_{0}(t)=\frac{i}{\hbar}\left[H_{0}^{(N)}+\Delta H_{0}^{(N)}(t)\right]+\lambda_{0}
$$

Here $\lambda_{0}$ is some real constant so that both $[S(t)]^{-1}$ and $\left[\tilde{S}_{0}\right]^{-1}$ exist, and the operators $\tilde{U}(t, s)$ and $\tilde{U}_{0}(t, s)$ are well defined and bounded [48]. Formally one has

$$
\frac{d}{d r} \tilde{U}(t, r) U(r, s)=-\tilde{U}(t, r)\left[\frac{d}{d r} S(r)\right][S(r)]^{-1} U(r, s) .
$$

Integrating this on the time $r$ from $s$ to $t$, one obtain ${ }^{10}$

$$
\tilde{U}(t, s)=U(t, s)+\int_{s}^{t} d r \tilde{U}(t, r)\left[\frac{d}{d r} S(r)\right][S(r)]^{-1} U(r, s) .
$$

Further one has

$$
[S(s)]^{-1}=\left[\tilde{S}_{0}(s)\right]^{-1}-[S(s)]^{-1} \frac{i}{\hbar} H_{\mathrm{per}}^{(N)}(s)\left[\tilde{S}_{0}(s)\right]^{-1} .
$$

Using these two formulas, the right-hand side of (3.15) can be evaluated as

$$
\begin{aligned}
& H_{\mathrm{per}}^{(N)}(s)[S(s)]^{-1} \tilde{U}^{(N)}(s,-T) S(-T) \Psi^{(N)}(-T) \\
= & H_{\mathrm{per}}^{(N)}(s)\left[\tilde{S}_{0}(s)\right]^{-1} U^{(N)}(s,-T) \tilde{S}_{0}(-T) \Psi^{(N)}(-T)+o(F) .
\end{aligned}
$$

\footnotetext{
${ }^{10}$ For simplicity we have given the formal derivation here although the resulting integral equation (3.22) is justified [48].
} 
Similarly one has

$$
\tilde{U}_{0}(t, s)=U_{0}(t, s)+\int_{s}^{t} d r \tilde{U}_{0}(t, r)\left[\frac{d}{d r} \tilde{S}_{0}(r)\right]\left[\tilde{S}_{0}(r)\right]^{-1} U_{0}(r, s) .
$$

Hence the right-hand side of (3.16) can be evaluated as

$$
\begin{aligned}
& H_{\mathrm{per}}^{(N)}(s)\left[\tilde{S}_{0}(s)\right]^{-1} \tilde{U}_{0}^{(N)}(s,-T) \tilde{S}_{0}(-T) \Psi^{(N)}(-T) \\
= & H_{\mathrm{per}}^{(N)}(s)\left[\tilde{S}_{0}(s)\right]^{-1} U_{0}^{(N)}(s,-T) \tilde{S}_{0}(-T) \Psi^{(N)}(-T)+o(F) .
\end{aligned}
$$

Combining (3.15), (3.16), (3.24) and (3.26), one has

$$
\begin{aligned}
& \left\|H_{\mathrm{per}}^{(N)}(s)\left[U^{(N)}(s,-T)-U_{0}^{(N)}(s,-T)\right] \Psi^{(N)}(-T)\right\| \\
= & \left\|H_{\mathrm{per}}^{(N)}(s)\left[\tilde{S}_{0}(s)\right]^{-1}\left[U^{(N)}(s,-T)-U_{0}^{(N)}(s,-T)\right] \tilde{S}_{0}(-T) \Psi^{(N)}(-T)\right\|+o(F) .
\end{aligned}
$$

This right-hand side is of $o(F)$ from Lemma 3.1 because the operator $H_{\text {per }}^{(N)}(s)\left[\tilde{S}_{0}(s)\right]^{-1}$ is bounded and already of order $F$.

We take the initial state $\omega_{0}$ at the time $t=t_{0}=-T$ as

$$
\omega_{0}(\cdots)=\frac{1}{q} \sum_{\mu=1}^{q}\left\langle\Phi_{0, \mu}^{(N)},\left(U_{0}^{(N)}(-T, 0)\right)^{\dagger}(\cdots) U_{0}^{(N)}(-T, 0), \Phi_{0, \mu}^{(N)}\right\rangle,
$$

where the ground state vectors $\Phi_{0, \mu}^{(N)}$ are normalized. Using the projection $Q\left(E_{0}^{(N)}\right)$ onto the sector of the ground state, we have

$$
\begin{aligned}
\omega_{0}(a) & =\frac{1}{q} \sum_{\mu=1}^{q}\left\langle\Phi_{0, \mu}^{(N)},\left(U_{0}^{(N)}(-T, 0)\right)^{\dagger} a U_{0}^{(N)}(-T, 0), \Phi_{0, \mu}^{(N)}\right\rangle \\
& =\frac{1}{q} \operatorname{Tr} Q\left(E_{0}^{(N)}\right)\left(U_{0}^{(N)}(-T, 0)\right)^{\dagger} a U_{0}^{(N)}(-T, 0) \\
& =\frac{1}{q} \operatorname{Tr} Q\left(E_{0}^{(N)}\right) a
\end{aligned}
$$

for any observable $a$ in the domain. Here Tr stands for the trace on the Hilbert space, and we have used the fact that $Q\left(E_{0}^{(N)}\right)$ commutes with the unitary operator $U_{0}^{(N)}(t, s)$. Starting from this initial state is physically natural as we discussed in the preceding section.

With this initial condition, the total current at time $t$ is given by

$$
\mathbf{j}_{\text {tot }}(t)=-\frac{e}{L_{x} L_{y}} \frac{1}{q} \sum_{\mu=1}^{q}\left\langle\Psi_{0, \mu}^{(N)}(t), \mathbf{v}_{\text {tot }}(t) \Psi_{0, \mu}^{(N)}(t)\right\rangle,
$$

where $\Psi_{0, \mu}^{(N)}(t)$ is the solution of the time-dependent Schrödinger equation (3.5) with the initial state $U_{0}^{(N)}(-T, 0) \Phi_{0, \mu}^{(N)}$ for $\mu=1,2, \ldots, q$, and the total velocity operator $\mathbf{v}_{\text {tot }}(t)$ is given by $(2.21)$. This total current $\mathbf{j}_{\text {tot }}(t)$ can be decomposed into two parts as

$$
\mathbf{j}_{\text {tot }}(t)=\mathbf{j}_{0}+\mathbf{j}_{\text {ind }}(t)
$$


with the current,

$$
\mathbf{j}_{0}=-\frac{e}{L_{x} L_{y}} \frac{1}{q} \sum_{\mu=1}^{q}\left\langle\Phi_{0, \mu}^{(N)},\left[U_{0}^{(N)}(t, 0)\right]^{\dagger} \mathbf{v}_{\mathrm{tot}}^{(0)} U_{0}^{(N)}(t, 0) \Phi_{0, \mu}^{(N)}\right\rangle
$$

where the velocity operator $\mathbf{v}_{\text {tot }}^{(0)}$ is given by (2.25). The current $\mathbf{j}_{0}$ of (3.32) is independent of the time $t$, and is equal to the initial current or the persistent current without the external electric field. Actually it can be rewritten as

$$
\mathbf{j}_{0}=-\frac{e}{L_{x} L_{y}} \frac{1}{q} \operatorname{Tr}\left[\mathbf{v}_{\text {tot }}^{(0)} Q\left(E_{0}^{(N)}\right)\right]
$$

in the same way as in the above.

Clearly the rest of the current, $\mathbf{j}_{\text {ind }}(t)=\left(j_{\text {ind, } x}(t), j_{\text {ind, } y}(t)\right)$, is the induced current by the external electric field. Using Theorem 3.2, the Hall current (the $x$ component of $\mathbf{j}_{\text {ind }}(t)$ ) induced by the electric field is given by

$$
\begin{aligned}
j_{\text {ind }, x}(t) & =\frac{e}{L_{x} L_{y}} \frac{i}{\hbar} \frac{1}{q} \sum_{\mu=1}^{q} \int_{-T}^{t} d s\left\langle U_{0}^{(N)}(t, 0) \Phi_{0, \mu}^{(N)}, v_{\text {tot }, x}^{(0)} U_{0}^{(N)}(t, s) H_{\text {per }}^{(N)}(s) U_{0}^{(N)}(s, 0) \Phi_{0, \mu}^{(N)}\right\rangle \\
& + \text { c.c. }+o(F) \\
& =\frac{e^{2}}{L_{x} L_{y}} \frac{i}{\hbar} \frac{1}{q} \sum_{\mu=1}^{q} \sum_{n \geq 1, \mu^{\prime}}\left\langle\Phi_{0, \mu}^{(N)}, v_{\text {tot }, x}^{(0)} \Phi_{n, \mu^{\prime}}^{(N)}\right\rangle\left\langle\Phi_{n, \mu^{\prime}}^{(N)}, v_{\text {tot }, y}^{(0)} \Phi_{0, \mu}^{(N)}\right\rangle \\
& \times \exp \left[\frac{i}{\hbar}\left(E_{0, \mu}^{(N)}-E_{n}^{(N)}\right) t\right] \int_{-T}^{t} d s \exp \left[-\frac{i}{\hbar}\left(E_{0, \mu}^{(N)}-E_{n}^{(N)}\right) s\right] \alpha(s)+\text { c.c. }+o(F),
\end{aligned}
$$

where $\Phi_{n, \mu^{\prime}}^{(N)}$ is the normalized eigenvector of the Hamiltonian $H_{0}^{(N)}$ with the energy eigenvalue $E_{n}^{(N)}$ for $n \geq 1$, and c.c. stands for the complex conjugate of the first part. Note that

$$
\begin{aligned}
& \frac{1}{F} \int_{-T}^{t} d s \exp \left[-\frac{i}{\hbar}\left(E_{0, \mu}^{(N)}-E_{n}^{(N)}\right) s\right] \alpha(s) \\
= & {\left[\frac{i \hbar T}{E_{0, \mu}^{(N)}-E_{n}^{(N)}+i \hbar \eta}-\frac{\hbar^{2}}{\left(E_{0, \mu}^{(N)}-E_{n}^{(N)}+i \hbar \eta\right)^{2}}\right] e^{-\eta T} \exp \left[i\left(E_{0, \mu}^{(N)}-E_{n}^{(N)}\right) T / \hbar\right] } \\
+ & \hbar^{2}\left[\frac{1}{\left(E_{0, \mu}^{(N)}-E_{n}^{(N)}\right)^{2}}-\frac{1}{\left(E_{0, \mu}^{(N)}-E_{n}^{(N)}+i \hbar \eta\right)^{2}}\right] \\
- & {\left[\frac{i \hbar t}{E_{0, \mu}^{(N)}-E_{n}^{(N)}}+\frac{\hbar^{2}}{\left(E_{0, \mu}^{(N)}-E_{n}^{(N)}\right)^{2}}\right] \exp \left[-i\left(E_{0, \mu}^{(N)}-E_{n}^{(N)}\right) t / \hbar\right] }
\end{aligned}
$$

for the time $t \geq 0$. In the following, we will consider only the time $t \geq 0$. Substituting (3.35) into (3.34), we obtain the linear response coefficient,

$$
\sigma_{\text {tot }, x y}(t ; \eta, T):=\lim _{F \rightarrow 0} \frac{j_{\text {ind }, x}(t)}{F}=\sigma_{x y}+\gamma_{x y} \cdot t+\delta \sigma_{x y}(t ; \eta, T),
$$


in the $x$ direction, where the first term which we call the Hall conductance is given by

$$
\sigma_{x y}=-\frac{i \hbar e^{2}}{L_{x} L_{y}} \frac{1}{q} \sum_{\mu=1}^{q} \sum_{n \geq 1, \mu^{\prime}}\left[\frac{\left\langle\Phi_{0, \mu}^{(N)}, v_{\mathrm{tot}, x}^{(0)} \Phi_{n, \mu^{\prime}}^{(N)}\right\rangle\left\langle\Phi_{n, \mu^{\prime}}^{(N)}, v_{\mathrm{tot}, y}^{(0)} \Phi_{0, \mu}^{(N)}\right\rangle}{\left(E_{0, \mu}^{(N)}-E_{n}^{(N)}\right)^{2}}-\text { c.c. }\right] \text {, }
$$

and the acceleration coefficient $\gamma_{x y}$ of the second term which is linear in the time $t$ is

$$
\gamma_{x y}=\frac{e^{2}}{L_{x} L_{y}} \frac{1}{q} \sum_{\mu=1}^{q} \sum_{n \geq 1, \mu^{\prime}}\left[\left\langle\Phi_{0, \mu}^{(N)}, v_{\mathrm{tot}, x}^{(0)} \Phi_{n, \mu^{\prime}}^{(N)}\right\rangle \frac{1}{E_{0, \mu}^{(N)}-E_{n}^{(N)}}\left\langle\Phi_{n, \mu^{\prime}}^{(N)}, v_{\mathrm{tot}, y}^{(0)} \Phi_{0, \mu}^{(N)}\right\rangle+\text { c.c. }\right] .
$$

The third term $\delta \sigma_{x y}(t ; \eta, T)$ is given by

$$
\delta \sigma_{x y}(t ; \eta, T)=\frac{i e^{2}}{L_{x} L_{y}} \frac{1}{q} \sum_{\mu=1}^{q} \sum_{n \geq 1, \mu^{\prime}}\left\langle\Phi_{0, \mu}^{(N)}, v_{\mathrm{tot}, x}^{(0)} \Phi_{n, \mu^{\prime}}^{(N)}\right\rangle\left\langle\Phi_{n, \mu^{\prime}}^{(N)}, v_{\mathrm{tot}, y}^{(0)} \Phi_{0, \mu}^{(N)}\right\rangle \mathcal{M}\left(t, \mathcal{E}_{0, \mu}^{n} ; \eta, T\right)+\text { c.c. }
$$

with $\mathcal{E}_{0, \mu}^{n}=E_{0, \mu}^{(N)}-E_{n}^{(N)}$ and

$$
\mathcal{M}(t, \mathcal{E} ; \eta, T)=\left\{\left[\frac{i T}{\mathcal{E}+i \hbar \eta}-\frac{\hbar}{(\mathcal{E}+i \hbar \eta)^{2}}\right] e^{-\eta T} e^{i \mathcal{E} T / \hbar}+\left[\frac{\hbar}{\mathcal{E}^{2}}-\frac{\hbar}{(\mathcal{E}+i \hbar \eta)^{2}}\right]\right\} e^{i \mathcal{E} t / \hbar}
$$

Similarly the induced current in the $y$ direction is

$$
\begin{aligned}
j_{\text {ind }, y}(t) & =\frac{N}{L_{x} L_{y}} \frac{e^{2}}{m_{e}} F t \\
& +\frac{e}{L_{x} L_{y}} \frac{i}{\hbar} \frac{1}{q} \sum_{\mu=1}^{q} \int_{-T}^{t} d s\left\langle U_{0}^{(N)}(t, 0) \Phi_{0, \mu}^{(N)}, v_{\text {tot }, y}^{(0)} U_{0}^{(N)}(t, s) H_{\mathrm{per}}^{(N)}(s) U_{0}^{(N)}(s, 0) \Phi_{0, \mu}^{(N)}\right\rangle \\
& + \text { c.c. }+o(F)
\end{aligned}
$$

and the linear response coefficient is given by

$$
\sigma_{\mathrm{tot}, y y}(t ; \eta, T):=\lim _{F \rightarrow 0} \frac{j_{\mathrm{ind}, y}(t)}{F}=\gamma_{y y} \cdot t+\delta \sigma_{y y}(t ; \eta, T),
$$

where

$$
\gamma_{y y}=\frac{e^{2}}{L_{x} L_{y}}\left[\frac{N}{m_{e}}+\frac{2}{q} \sum_{\mu=1}^{q} \sum_{n \geq 1, \mu^{\prime}}\left\langle\Phi_{0, \mu}^{(N)}, v_{\mathrm{tot}, y}^{(0)} \Phi_{n, \mu^{\prime}}^{(N)}\right\rangle \frac{1}{E_{0, \mu}^{(N)}-E_{n}^{(N)}}\left\langle\Phi_{n, \mu^{\prime}}^{(N)}, v_{\mathrm{tot}, y}^{(0)} \Phi_{0, \mu}^{(N)}\right\rangle\right]
$$

and $\delta \sigma_{y y}(t ; \eta, T)$ is given by replacing the velocity operator $v_{\text {tot }, x}^{(0)}$ with $v_{\text {tot }, y}^{(0)}$ in $\delta \sigma_{x y}(t ; \eta, T)$.

\section{The system with translation invariance}

In this section, we check the validity of our linear response formulas in the special case without the vector potential $\mathbf{A}_{\mathrm{P}}$ and without the electrostatic potential $W$. 
The Hamiltonian without the external electric field is given by

$$
H_{0}^{(N)}=\sum_{j=1}^{N} \frac{1}{2 m_{e}}\left\{\left(p_{x, j}-e B y_{j}+\phi_{x}\right)^{2}+\left(p_{y, j}+\phi_{y}\right)^{2}\right\}+\sum_{1 \leq i<j \leq N} W^{(2)}\left(\mathbf{r}_{i}-\mathbf{r}_{j}\right) .
$$

Clearly the system has translation invariance [37] in both $x$ and $y$ directions because the electron-electron interaction $W^{(2)}$ is a function of the relative coordinate only. We assume $W^{(2)} \in C^{1}\left(\mathbf{R}^{2}\right)$. In this situation with $B \neq 0$, the well-known results are obtained as

$$
\sigma_{x y}=-\frac{e^{2}}{h} \nu
$$

and

$$
\gamma_{x y}=\gamma_{y y}=0
$$

where $\nu$ is the filling factor of the Landau level, i.e., $\nu=N / M$ with the number $N$ of the electrons and with the number $M$ of the single electron states in a single Landau level without the electron-electron interaction. Further we prove the bounds,

$$
\left|\delta \sigma_{s y}\right| \leq \frac{e^{2}}{h} \nu\left(1+\omega_{c} T\right) e^{-\eta T}+\frac{e^{2}}{h} \nu\left(2+\frac{\eta}{\omega_{c}}\right) \frac{\eta}{\omega_{c}} \quad \text { for } s=x, y .
$$

Clearly the first term in the right-hand side vanishes in the large limit of $T$, and the second term also vanishes in the small limit of $\eta$. Further we also stress that the right-hand side is independent of the system sizes, $L_{x}, L_{y}$, and of the number $N$ of the electrons for a fixed filling factor $\nu$.

In order to give proofs of (4.2) and (4.3), we note that

$$
\begin{gathered}
v_{\mathrm{tot}, x}^{(0)}=-\frac{i m_{e}}{\hbar e B}\left[v_{\mathrm{tot}, y}^{(0)}, H_{0}^{(N)}\right], \\
v_{\mathrm{tot}, y}^{(0)}=\frac{i m_{e}}{\hbar e B}\left[v_{\mathrm{tot}, x}^{(0)}, H_{0}^{(N)}\right]
\end{gathered}
$$

and

$$
\frac{i m_{e}}{\hbar e B}\left[v_{\text {tot }, x}^{(0)}, v_{\text {tot }, y}^{(0)}\right]=\frac{N}{m_{e}} .
$$

Let $\Phi$ be a vector in the domain of $H_{0}^{(N)}$, i.e., $\left\|H_{0}^{(N)} \Phi\right\|<\infty$. Then one has

$$
\begin{aligned}
\left\langle\left(E_{0, \mu}^{(N)}-H_{0}^{(N)}\right) \Phi, v_{\text {tot }, x}^{(0)} \Phi_{0, \mu}^{(N)}\right\rangle & =\left\langle v_{\text {tot }, x}^{(0)} \Phi, H_{0}^{(N)} \Phi_{0, \mu}^{(N)}\right\rangle-\left\langle H_{0}^{(N)} \Phi, v_{\text {tot }, x}^{(0)} \Phi_{0, \mu}^{(N)}\right\rangle \\
& =\lim _{n \rightarrow \infty}\left(\left\langle v_{\text {tot }, x}^{(0)} \Phi, H_{0}^{(N)} \Psi_{\mu}^{(n)}\right\rangle-\left\langle H_{0}^{(N)} \Phi, v_{\text {tot }, x}^{(0)} \Psi_{\mu}^{(n)}\right\rangle\right) \\
& =\lim _{n \rightarrow \infty}\left\langle\Phi,\left[v_{\text {tot }, x}^{(0)}, H_{0}^{(N)}\right] \Psi_{\mu}^{(n)}\right\rangle \\
& =\frac{\hbar e B}{i m_{e}}\left\langle\Phi, v_{\text {tot }, y}^{(0)} \Phi_{0, \mu}^{(N)}\right\rangle
\end{aligned}
$$

where $\Psi_{\mu}^{(n)} \in C^{\infty}(\mathcal{T})$ is an approximate vector ${ }^{11}$ such that $\left\|\left(H_{0}^{(N)}+\lambda\right)\left(\Psi_{\mu}^{(n)}-\Phi_{0, \mu}^{(N)}\right)\right\| \rightarrow 0$ as $n \rightarrow \infty$ with a positive constant $\lambda$ satisfying $H_{0}^{(N)}+\lambda>0$, and we have used the

\footnotetext{
${ }^{11}$ One can easily find such a vector $\Psi_{\mu}^{(n)}$ by using the Fourier expansion in terms of the eigenvectors (6.7) of the single-electron Landau Hamiltonian in Section 6.1 below.
} 
commutation relation (4.6). Using this and the commutation relation (4.7), one has

$$
\begin{aligned}
& \frac{2}{q} \sum_{\mu=1}^{q}\left\langle\Phi_{0, \mu}^{(N)}, v_{\text {tot }, y}^{(0)} \frac{1-Q\left(E_{0}^{(N)}\right)}{E_{0, \mu}^{(N)}-H_{0}^{(N)}} v_{\text {tot }, y}^{(0)} \Phi_{0, \mu}^{(N)}\right\rangle \\
= & \frac{1}{q} \sum_{\mu=1}^{q} \frac{i m_{e}}{\hbar e B}\left\langle\Phi_{0, \mu}^{(N)}, v_{\text {tot }, y}^{(0)}\left[1-Q\left(E_{0}^{(N)}\right)\right] v_{\text {tot }, x}^{(0)} \Phi_{0, \mu}^{(N)}\right\rangle+\text { c.c. } \\
= & \frac{1}{q} \sum_{\mu=1}^{q} \frac{i m_{e}}{\hbar e B}\left\langle\Phi_{0, \mu}^{(N)},\left[v_{\text {tot }, y}^{(0)}, v_{\text {tot }, x}^{(0)}\right] \Phi_{0, \mu}^{(N)}\right\rangle=-\frac{N}{m_{e}} .
\end{aligned}
$$

Substituting this into the expression (3.43) of $\gamma_{y y}$, one gets $\gamma_{y y}=0$. In the same way, one can easily obtain $\gamma_{x y}=0$ by using the expression (3.38) for $\gamma_{x y}$. For the rest of the Hall conductance $\sigma_{x y}$ of (4.2), one has

$$
\begin{aligned}
& \frac{1}{q} \sum_{\mu=1}^{q}\left\langle\Phi_{0, \mu}^{(N)}, v_{\mathrm{tot}, x}^{(0)} \frac{1-Q\left(E_{0}^{(N)}\right)}{\left(E_{0, \mu}^{(N)}-H_{0}^{(N)}\right)^{2}} v_{\mathrm{tot}, y}^{(0)} \Phi_{0, \mu}^{(N)}\right\rangle-\text { c.c. } \\
= & -\frac{1}{q} \sum_{\mu=1}^{q}\left(\frac{m_{e}}{\hbar e B}\right)^{2}\left\langle\Phi_{0, \mu}^{(N)}, v_{\mathrm{tot}, y}^{(0)}\left[1-Q\left(E_{0}^{(N)}\right)\right] v_{\mathrm{tot}, x}^{(0)} \Phi_{0, \mu}^{(N)}\right\rangle+\text { c.c. } \\
= & \frac{1}{q} \sum_{\mu=1}^{q}\left(\frac{m_{e}}{\hbar e B}\right)^{2}\left\langle\Phi_{0, \mu}^{(N)},\left[v_{\mathrm{tot}, x}^{(0)}, v_{\mathrm{tot}, y}^{(0)}\right] \Phi_{0, \mu}^{(N)}\right\rangle=-\frac{i N}{\hbar e B} .
\end{aligned}
$$

Substituting this into the expression (3.37) of $\sigma_{x y}$, the desired result (4.2) is obtained.

Next let us give a proof of the bounds (4.4). To this end, we recall the expression of $\delta \sigma_{s y}(t ; \eta, T)$ as

$$
\delta \sigma_{s y}(t ; \eta, T)=\frac{i e^{2}}{L_{x} L_{y}} \frac{1}{q} \sum_{\mu=1}^{q} \sum_{n \geq 1, \mu^{\prime}}\left\langle\Phi_{0, \mu}^{(N)}, v_{\mathrm{tot}, s}^{(0)} \Phi_{n, \mu^{\prime}}^{(N)}\right\rangle\left\langle\Phi_{n, \mu^{\prime}}^{(N)}, v_{\mathrm{tot}, y}^{(0)} \Phi_{0, \mu}^{(N)}\right\rangle \mathcal{M}\left(t, \mathcal{E}_{0, \mu}^{n} ; \eta, T\right)+\text { c.c. }
$$

with $\mathcal{E}_{0, \mu}^{n}=E_{0, \mu}^{(N)}-E_{n}^{(N)}$ and

$$
\mathcal{M}(t, \mathcal{E} ; \eta, T)=\left\{\left[\frac{i T}{\mathcal{E}+i \hbar \eta}-\frac{\hbar}{(\mathcal{E}+i \hbar \eta)^{2}}\right] e^{-\eta T} e^{i \mathcal{E} T / \hbar}+\left[\frac{\hbar}{\mathcal{E}^{2}}-\frac{\hbar}{(\mathcal{E}+i \hbar \eta)^{2}}\right]\right\} e^{i \mathcal{E} t / \hbar} .
$$

For a generic filling factor $\nu$, we cannot expect the existence of a system-size-independent energy gap above the sector of the ground state. Namely the gap might become small for a large volume of the system. Therefore we must treat carefully the denominators of the fractions appeared in the expression of $\mathcal{M}(t, \mathcal{E} ; \eta, T)$.

To begin with, we note that

$$
\frac{1}{\mathcal{E}^{2}}-\frac{1}{(\mathcal{E}+i \hbar \eta)^{2}}=\frac{2 i \hbar \eta}{\mathcal{E}(\mathcal{E}+i \hbar \eta)^{2}}-\frac{\hbar^{2} \eta^{2}}{\mathcal{E}^{2}(\mathcal{E}+i \hbar \eta)^{2}}
$$

Using this identity, $L_{x} L_{y}=2 \pi M \ell_{B}^{2}$ and $\nu=N / M$, we have

$$
\delta \sigma_{s y}(t ; \eta, T)=i \frac{e^{2}}{h} \nu\left[\left(A_{1}+A_{2} \omega_{c} T\right) e^{-\eta T}+\left(2 A_{3}+A_{4} \eta / \omega_{c}\right) \eta / \omega_{c}\right]+\text { c.c. }
$$


where

$$
A_{j}=\frac{\hbar e B}{N} \frac{1}{q} \sum_{\mu=1}^{q} \sum_{n \geq 1 ; \mu^{\prime}}\left\langle\Phi_{0, \mu}^{(N)}, v_{\mathrm{tot}, s}^{(0)} \Phi_{n, \mu^{\prime}}^{(N)}\right\rangle \mathcal{M}_{j}\left(\mathcal{E}_{0, \mu}^{n}\right)\left\langle\Phi_{n, \mu^{\prime}}^{(N)}, v_{\mathrm{tot}, y}^{(0)} \Phi_{0, \mu}^{(N)}\right\rangle
$$

with

$$
\begin{gathered}
\mathcal{M}_{1}(\mathcal{E})=-\frac{1}{(\mathcal{E}+i \hbar \eta)^{2}} \exp [i \mathcal{E}(t+T) / \hbar] \\
\mathcal{M}_{2}(\mathcal{E})=\frac{i}{\hbar \omega_{c}} \frac{1}{\mathcal{E}+i \hbar \eta} \exp [i \mathcal{E}(t+T) / \hbar] \\
\mathcal{M}_{3}(\mathcal{E})=\frac{i \hbar \omega_{c}}{\mathcal{E}(\mathcal{E}+i \hbar \eta)^{2}} e^{i \mathcal{E} t / \hbar}
\end{gathered}
$$

and

$$
\mathcal{M}_{4}(\mathcal{E})=-\frac{\hbar^{2} \omega_{c}^{2}}{\mathcal{E}^{2}(\mathcal{E}+i \hbar \eta)^{2}} e^{i \mathcal{E} t / \hbar}
$$

We can prove $\left|A_{j}\right| \leq 1 / 2$ for $j=1,2,3,4$. The desired bound (4.4) follows from these bounds for $A_{j}$. Since all of $A_{j}$ can be treated in the same way, we shall give a proof for $\left|A_{1}\right| \leq 1 / 2$ only. In the same way as the above, one has

$$
\begin{aligned}
A_{1} & =-\frac{\hbar e B}{N} \frac{1}{q} \sum_{\mu=1}^{q}\left\langle\Phi_{0 \mu}^{(N)}, v_{\mathrm{tot}, s}^{(0)} e^{i \hat{\theta}} \frac{1-Q\left(E_{0}^{(N)}\right)}{\left(E_{0, \mu}^{(N)}-H_{0}^{(N)}+i \hbar \eta\right)^{2}} v_{\mathrm{tot}, y}^{(0)} \Phi_{0, \mu}^{(N)}\right\rangle \\
& =-\frac{i m_{e}}{N} \frac{1}{q} \sum_{\mu=1}^{q}\left\langle\Phi_{0 \mu}^{(N)}, v_{\mathrm{tot}, s}^{(0)} e^{i \hat{\theta}} \frac{1-Q\left(E_{0}^{(N)}\right)}{\left(E_{0, \mu}^{(N)}-H_{0}^{(N)}+i \hbar \eta\right)^{2}}\left(E_{0, \mu}^{(N)}-H_{0}^{(N)}\right) v_{\mathrm{tot}, x}^{(0)} \Phi_{0, \mu}^{(N)}\right\rangle \\
& =-\frac{m_{e}^{2}}{\hbar e B N} \frac{1}{q} \sum_{\mu=1}^{q}\left\langle\Phi_{0 \mu}^{(N)}, v_{\mathrm{tot}, s}^{(0)} e^{i \hat{\theta}} \frac{1-Q\left(E_{0}^{(N)}\right)}{\left(E_{0, \mu}^{(N)}-H_{0}^{(N)}+i \hbar \eta\right)^{2}}\left(E_{0, \mu}^{(N)}-H_{0}^{(N)}\right)^{2} v_{\mathrm{tot}, y}^{(0)} \Phi_{0, \mu}^{(N)}\right\rangle,
\end{aligned}
$$

where we have written $\hat{\theta}=\left(E_{0, \mu}^{(N)}-H_{0}^{(N)}\right)(t+T) / \hbar$. Let $\Phi$ be a vector in the domain of $v_{\text {tot }, x}^{(0)}$. Then one has

$$
\begin{aligned}
\left\langle\Phi,\left[1-Q\left(E_{0}^{(N)}\right)\right] v_{\text {tot }, y}^{(0)} \Phi_{0, \mu}^{(N)}\right\rangle & =-\frac{i m_{e}}{\hbar e B} \lim _{n \rightarrow \infty}\left\langle\Phi,\left[1-Q\left(E_{0}^{(N)}\right)\right]\left[H_{0}^{(N)}, v_{\text {tot }, x}^{(0)}\right] \Psi_{\mu}^{(n)}\right\rangle \\
& =-\frac{i m_{e}}{\hbar e B} \lim _{n \rightarrow \infty}\left\langle\Phi,\left[1-Q\left(E_{0}^{(N)}\right)\right]\left(H_{0}^{(N)}-E_{0, \mu}^{(N)}\right) v_{\text {tot }, x}^{(0)} \Psi_{\mu}^{(n)}\right\rangle
\end{aligned}
$$

where $\Psi_{\mu}^{(n)}$ is the approximate vector which was introduced for proving (4.8), and we have used the commutation relation (4.6). Substituting this into the expression (4.20) of $A_{1}$ and then applying the Schwarz inequality, we obtain

$$
\left|A_{1}\right| \leq \frac{m_{e}^{3}}{(\hbar e B)^{2} N} \max _{s} \lim _{n \rightarrow \infty} \frac{1}{q} \sum_{\mu=1}^{q}\left\langle v_{\mathrm{tot}, s}^{(0)} \Psi_{\mu}^{(n)},\left[1-Q\left(E_{0}^{(N)}\right)\right]\left(H_{0}^{(N)}-E_{0, \mu}^{(N)}\right) v_{\mathrm{tot}, s}^{(0)} \Psi_{\mu}^{(n)}\right\rangle .
$$


Note that, for a symmetric operator $A$, one has formally

$$
\begin{aligned}
& \sum_{\mu=1}^{q}\left\langle A \Phi_{0, \mu}^{(N)},\left[1-Q\left(E_{0}^{(N)}\right)\right]\left(H_{0}^{(N)}-E_{0, \mu}^{(N)}\right) A \Phi_{0, \mu}^{(N)}\right\rangle \\
= & \sum_{\mu=1}^{q}\left\langle A \Phi_{0, \mu}^{(N)},\left(H_{0}^{(N)}-E_{0, \mu}^{(N)}\right) A \Phi_{0, \mu}^{(N)}\right\rangle \\
= & \frac{1}{2} \sum_{\mu=1}^{q}\left\{\left\langle A \Phi_{0, \mu}^{(N)},\left(H_{0}^{(N)}-E_{0, \mu}^{(N)}\right) A \Phi_{0, \mu}^{(N)}\right\rangle+\left\langle\left(H_{0}^{(N)}-E_{0, \mu}^{(N)}\right) A \Phi_{0, \mu}^{(N)}, A \Phi_{0, \mu}^{(N)}\right\rangle\right\} \\
= & \frac{1}{2} \sum_{\mu=1}^{q}\left\{\left\langle A \Phi_{0, \mu}^{(N)},\left[H_{0}^{(N)}, A\right] \Phi_{0, \mu}^{(N)}\right\rangle+\left\langle\left[H_{0}^{(N)}, A\right] \Phi_{0, \mu}^{(N)}, A \Phi_{0, \mu}^{(N)}\right\rangle\right\} \\
= & \frac{1}{2} \sum_{\mu=1}^{q}\left\langle\Phi_{0, \mu}^{(N)},\left[A,\left[H_{0}^{(N)}, A\right]\right] \Phi_{0, \mu}^{(N)}\right\rangle
\end{aligned}
$$

where we have used

$$
\sum_{\mu=1}^{q} \sum_{\mu^{\prime}=1}^{q}\left\langle A \Phi_{0, \mu}^{(N)}, \Phi_{0, \mu^{\prime}}^{(N)}\right\rangle\left(E_{0, \mu^{\prime}}^{(N)}-E_{0, \mu}^{(N)}\right)\left\langle\Phi_{0, \mu^{\prime}}^{(N)}, A \Phi_{0, \mu}^{(N)}\right\rangle=0 .
$$

We can justify this formal identity (4.23) for $A=v_{\text {tot }, s}^{(0)}$ by using the approximate vector $\Psi_{\mu}^{(n)}$. Combining this observation, the commutation relations, (4.5), (4.6), (4.7), and the bound (4.22), we obtain $\left|A_{1}\right| \leq 1 / 2$.

In the case with $B=0$, one has

$$
\sigma_{x y}=\delta \sigma_{x y}(t ; \eta, T)=\delta \sigma_{y y}(t ; \eta, T)=0
$$

and

$$
\gamma_{x y}=0, \quad \gamma_{y y}=\frac{N}{L_{x} L_{y}} \frac{e^{2}}{m_{e}}
$$

under the same assumptions as in the above. Clearly these imply that the total velocity of the electrons is proportional to the time $t$. The derivation is not hard as follows: From the assumptions, we have

$$
\left[v_{\text {tot }, s}^{(0)}, H_{0}^{(N)}\right]=0 \quad \text { for } s=x, y, \quad \text { and } \quad\left[v_{\text {tot }, x}^{(0)}, v_{\text {tot }, y}^{(0)}\right]=0 .
$$

Namely all of these operators commute with each other. This implies that all of the matrix elements in the expressions (3.37), (3.38), (3.43) and (4.11) of the coefficients vanishes. As a result, we get the desired results.

\section{The linear response coefficients averaged over the gauge parameters}

In this section, we treat the averaged Hall conductance $\overline{\sigma_{x y}(\phi)}$ of $(2.29)$ and the averaged acceleration coefficients $\overline{\gamma_{s y}(\boldsymbol{\phi})}$ of $(2.30)$. As is well known, the "topological" argument 
$[5,6,8,11,14,19,20,26,27]$ yields the integral and fractional quantization of the Hall conductance under the assumption on the excitation energy gap above the ground state. Following the argument, the fractional quantization (2.34) of the averaged Hall conductance $\overline{\sigma_{x y}(\phi)}$ will be proved in the most general setting of the present paper. In addition, we will prove that the averaged acceleration coefficients are exactly vanishing, i.e., $\overline{\gamma_{s y}(\phi)}=0$ for $s=x, y$. Thus we will give the proof of Theorem 2.2 in Section 5.1 below. In Section 5.2, we will also discuss the geometric property of the averaged Hall conductance $\overline{\sigma_{x y}(\phi)}$ as a geometric invariant for non-Abelian gauge fields on the gauge torus $\mathcal{T}_{\mathrm{g}}$. In particular, the integer $\mathcal{I}$ of the fractional quantized Hall conductance (2.31) is equal to an index of a Pauli-Dirac operator coupled to the gauge fields. In other words, the Hall conductance of the interacting electron gas is closely related to the ground state property of a single electron system coupled to the gauge fields.

The Hamiltonian of the quantum Hall system without the external electric field is given by

$$
H_{0}^{(N)}(\boldsymbol{\phi})=\sum_{j=1}^{N}\left\{\frac{1}{2 m_{e}}\left[\mathbf{p}_{j}+e \mathbf{A}\left(\mathbf{r}_{j}\right)+\phi\right]^{2}+W\left(\mathbf{r}_{j}\right)\right\}+\sum_{1 \leq i<j \leq N} W^{(2)}\left(\mathbf{r}_{i}-\mathbf{r}_{j}\right) .
$$

Since the gauge parameters $\phi=\left(\phi_{x}, \phi_{y}\right)$ play an important role in the following proof, we write the parameter dependence explicitly as $\Phi_{0, \mu}^{(N)}=\Phi_{0, \mu}^{(N)}(\phi)$ and $E_{0, \mu}^{(N)}=E_{0, \mu}^{(N)}(\phi)$ for the (quasi)degenerate ground state, and $\Phi_{n, \mu}^{(N)}=\Phi_{n, \mu}^{(N)}(\phi)$ and $E_{n}^{(N)}=E_{n}^{(N)}(\phi)$ for the excited states throughout this section. We also write the velocity operator as

$$
\mathbf{v}_{\text {tot }}^{(0)}(\boldsymbol{\phi})=\sum_{j=1}^{N} \frac{1}{m_{e}}\left[\mathbf{p}_{j}+e \mathbf{A}\left(\mathbf{r}_{j}\right)+\boldsymbol{\phi}\right] .
$$

Further, throughout this section, we require Assumption 2.1 which we need for the proof of Theorem 2.2.

\subsection{Proof of Theorem 2.2}

To begin with, we prepare some tools to rewrite the expressions of $\sigma_{x y}(\phi)$ and $\gamma_{s y}(\phi)$. The derivative of the projection $Q\left(E_{0}^{(N)}(\phi)\right)$ onto the sector of the ground state becomes

$$
\begin{aligned}
Q_{i}\left(E_{0}^{(N)}(\phi)\right):=\frac{\partial}{\partial \phi_{i}} Q\left(E_{0}^{(N)}(\phi)\right) & =\frac{\partial}{\partial \phi_{i}} \frac{1}{2 \pi i} \int_{\Gamma} d z \frac{1}{z-H_{0}^{(N)}(\phi)} \\
& =\frac{1}{2 \pi i} \int_{\Gamma} d z \frac{1}{z-H_{0}^{(N)}(\phi)} v_{\text {tot }, i}^{(0)}(\phi) \frac{1}{z-H_{0}^{(N)}(\phi)}
\end{aligned}
$$

where we have used the integral representation of the projection

$$
Q\left(E_{0}^{(N)}(\phi)\right)=\frac{1}{2 \pi i} \int_{\Gamma} d z \frac{1}{z-H_{0}^{(N)}(\phi)}
$$

with the resolvent $\left[z-H_{0}^{(N)}(\phi)\right]^{-1}$. Here the closed path $\Gamma$ encircles all of the ground state energy eigenvalues $E_{0, \mu}^{(N)}(\phi)$ which are isolated from the rest of the spectrum. We 
can take the path $\Gamma$ to be independent of $\phi$ because of Assumption 2.1. Using the operator $Q_{i}\left(E_{0}^{(N)}(\phi)\right)$, the Hall conductance $(3.37)$ can be rewritten as

$$
\sigma_{x y}(\boldsymbol{\phi})=-\frac{i \hbar e^{2}}{L_{x} L_{y}} \frac{1}{q} \operatorname{Tr} Q\left(E_{0}^{(N)}(\boldsymbol{\phi})\right)\left[Q_{x}\left(E_{0}^{(N)}(\boldsymbol{\phi})\right), Q_{y}\left(E_{0}^{(N)}(\boldsymbol{\phi})\right)\right],
$$

and for $\gamma_{x y}$ of (3.38) and $\gamma_{y y}$ of (3.43), we have

$$
\gamma_{x y}(\boldsymbol{\phi})=\frac{e^{2}}{L_{x} L_{y}} \frac{1}{q} \operatorname{Tr} v_{\text {tot }, x}^{(0)}(\phi)\left[Q_{y}\left(E_{0}^{(N)}(\phi)\right) Q\left(E_{0}^{(N)}(\phi)\right)+Q\left(E_{0}^{(N)}(\phi)\right) Q_{y}\left(E_{0}^{(N)}(\boldsymbol{\phi})\right)\right]
$$

and

$$
\begin{aligned}
& \gamma_{y y}(\boldsymbol{\phi}) \\
= & \frac{e^{2}}{L_{x} L_{y}}\left\{\frac{N}{m_{e}}+\frac{1}{q} \operatorname{Tr} v_{\mathrm{tot}, y}^{(0)}(\boldsymbol{\phi})\left[Q_{y}\left(E_{0}^{(N)}(\boldsymbol{\phi})\right) Q\left(E_{0}^{(N)}(\boldsymbol{\phi})\right)+Q\left(E_{0}^{(N)}(\boldsymbol{\phi})\right) Q_{y}\left(E_{0}^{(N)}(\boldsymbol{\phi})\right)\right]\right\}
\end{aligned}
$$

where Tr stands for the trace on the Hilbert space. The expression of the Hall conductance with the use of the derivative of a projection was used in refs. $[14,20,27]$. Further $\gamma_{s y}(\phi)$ can be rewritten as

$$
\gamma_{s y}(\phi)=\frac{e^{2}}{L_{x} L_{y}} \frac{1}{q} \frac{\partial}{\partial \phi_{y}} \operatorname{Tr} v_{\text {tot }, s}^{(0)}(\phi) Q\left(E_{0}^{(N)}(\phi)\right) \quad \text { for } s=x, y,
$$

where we have used the identity,

$$
Q_{s}\left(E_{0}^{(N)}(\phi)\right)=Q_{s}\left(E_{0}^{(N)}(\phi)\right) Q\left(E_{0}^{(N)}(\phi)\right)+Q\left(E_{0}^{(N)}(\phi)\right) Q_{s}\left(E_{0}^{(N)}(\phi)\right), \quad \text { for } s=x, y,
$$

which is derived by differentiation of $Q\left(E_{0}^{(N)}(\phi)\right)=Q\left(E_{0}^{(N)}(\phi)\right)^{2}$. We also note that

$$
Q\left(E_{0}^{(N)}(\phi)\right) Q_{s}\left(E_{0}^{(N)}(\phi)\right) Q\left(E_{0}^{(N)}(\phi)\right)=0 \quad \text { for } s=x, y,
$$

which is easily derived from (5.9).

Proposition 5.1 Under Assumption 2.1, there exist orthonormal vectors $\hat{\Phi}_{0, \mu}^{(N)}(\phi), \mu=$ $1,2, \ldots, q$ such that the sector of the (quasi)degenerate ground state is spanned by the vectors $\hat{\Phi}_{0, \mu}^{(N)}(\phi), \mu=1,2, \ldots, q$, and that all the vectors $\hat{\Phi}_{0, \mu}^{(N)}(\phi), \mu=1,2, \ldots, q$, are infinitely differentiable with respect to the gauge parameters $\boldsymbol{\phi}$ on the gauge torus $\mathcal{T}_{\mathrm{g}}$.

This proposition is essentially due to T. Kato. But, in his book [47], he treated only the case with a single variable. For reader's convenience, we give the proof of Proposition 5.1 in Appendix A along his line although the extension to two variables is not so difficult. In terms of these vectors $\hat{\Phi}_{0, \mu}^{(N)}(\boldsymbol{\phi})$, the acceleration coefficients $\gamma_{s y}(\boldsymbol{\phi})$ of $(5.8)$ are written as

$$
\gamma_{s y}(\phi)=\frac{e^{2}}{L_{x} L_{y}} \frac{1}{q} \sum_{\mu=1}^{q} \frac{\partial}{\partial \phi_{y}}\left\langle\hat{\Phi}_{0, \mu}^{(N)}(\phi), v_{\mathrm{tot}, s}^{(0)}(\phi) \hat{\Phi}_{0, \mu}^{(N)}(\phi)\right\rangle \quad \text { for } s=x, y .
$$


Next let us rewrite the Hall conductance $\sigma_{x y}(\phi)$ of $(5.5)$ in terms of the vectors $\hat{\Phi}_{0, \mu}^{(N)}(\phi)$. Note that

$$
\begin{aligned}
\frac{\partial}{\partial \phi_{i}} \hat{\Phi}_{0, \mu}^{(N)}(\boldsymbol{\phi}) & =\frac{\partial}{\partial \phi_{i}} Q\left(E_{0}^{(N)}(\boldsymbol{\phi})\right) \hat{\Phi}_{0, \mu}^{(N)}(\boldsymbol{\phi}) \\
& =Q_{i}\left(E_{0}^{(N)}(\boldsymbol{\phi})\right) \hat{\Phi}_{0, \mu}^{(N)}(\boldsymbol{\phi})+Q\left(E_{0}^{(N)}(\boldsymbol{\phi})\right) \frac{\partial}{\partial \phi_{i}} \hat{\Phi}_{0, \mu}^{(N)}(\boldsymbol{\phi})
\end{aligned}
$$

Therefore one has

$$
\left[1-Q\left(E_{0}^{(N)}(\phi)\right)\right] \frac{\partial}{\partial \phi_{i}} \hat{\Phi}_{0, \mu}^{(N)}(\phi)=Q_{i}\left(E_{0}^{(N)}(\phi)\right) \hat{\Phi}_{0, \mu}^{(N)}(\phi) .
$$

Using this identity (5.13), the Hall conductance $\sigma_{x y}(\phi)$ of $(5.5)$ can be written as

$$
\begin{aligned}
\sigma_{x y}(\boldsymbol{\phi}) & =-\frac{i \hbar e^{2}}{L_{x} L_{y}} \frac{1}{q} \sum_{\mu=1}^{q}\left[\left\langle\frac{\partial}{\partial \phi_{x}} \hat{\Phi}_{0, \mu}^{(N)}(\boldsymbol{\phi}),\left[1-Q\left(E_{0}^{(N)}(\boldsymbol{\phi})\right)\right] \frac{\partial}{\partial \phi_{y}} \hat{\Phi}_{0, \mu}^{(N)}(\boldsymbol{\phi})\right\rangle-(x \leftrightarrow y)\right] \\
& =-\frac{i \hbar e^{2}}{L_{x} L_{y}} \frac{1}{q} \sum_{\mu=1}^{q}\left[\left\langle\frac{\partial}{\partial \phi_{x}} \hat{\Phi}_{0, \mu}^{(N)}(\boldsymbol{\phi}), \frac{\partial}{\partial \phi_{y}} \hat{\Phi}_{0, \mu}^{(N)}(\boldsymbol{\phi})\right\rangle-(x \leftrightarrow y)\right] \\
& =-\frac{i \hbar e^{2}}{L_{x} L_{y}} \frac{1}{q} \sum_{\mu=1}^{q}\left[\frac{\partial}{\partial \phi_{x}}\left\langle\hat{\Phi}_{0, \mu}^{(N)}(\boldsymbol{\phi}), \frac{\partial}{\partial \phi_{y}} \hat{\Phi}_{0, \mu}^{(N)}(\boldsymbol{\phi})\right\rangle-(x \leftrightarrow y)\right]
\end{aligned}
$$

where we have used the identity

$$
\begin{aligned}
& \left\langle\frac{\partial}{\partial \phi_{y}} \hat{\Phi}_{0, \mu}^{(N)}(\phi), \hat{\Phi}_{0, \mu^{\prime}}^{(N)}(\phi)\right\rangle+\left\langle\hat{\Phi}_{0, \mu}^{(N)}(\phi), \frac{\partial}{\partial \phi_{y}} \hat{\Phi}_{0, \mu^{\prime}}^{(N)}(\phi)\right\rangle \\
= & \frac{\partial}{\partial \phi_{y}}\left\langle\hat{\Phi}_{0, \mu}^{(N)}(\boldsymbol{\phi}), \hat{\Phi}_{0, \mu^{\prime}}^{(N)}(\boldsymbol{\phi})\right\rangle=\frac{\partial}{\partial \phi_{y}} \delta_{\mu, \mu^{\prime}}=0
\end{aligned}
$$

for getting the second equality. The Hall conductance expressed in terms of the derivative of wavefunctions was first introduced in ref. [5].

Following Kunz [11], we shall introduce a gauge transformation as

$$
\tilde{\Phi}_{0, \mu}^{(N)}(\phi)=G^{(N)}(\phi) \hat{\Phi}_{0, \mu}^{(N)}(\phi)
$$

with

$$
G^{(N)}(\phi)=\prod_{j=1}^{N} \exp \left[\frac{i}{\hbar}\left(x_{j} \phi_{x}+y_{j} \phi_{y}\right)\right] .
$$

Then the Hamiltonian is transformed as

$$
\begin{aligned}
\tilde{H}_{0}^{(N)}(\boldsymbol{\phi}) & :=G^{(N)}(\boldsymbol{\phi}) H_{0}^{(N)}(\boldsymbol{\phi})\left[G^{(N)}(\boldsymbol{\phi})\right]^{-1} \\
& =\sum_{j=1}^{N}\left\{\frac{1}{m_{e}}\left[\mathbf{p}_{j}+e \mathbf{A}\left(\mathbf{r}_{j}\right)\right]^{2}+W\left(\mathbf{r}_{j}\right)\right\}+\sum_{1 \leq i<j \leq N} W^{(2)}\left(\mathbf{r}_{i}-\mathbf{r}_{j}\right) .
\end{aligned}
$$

The expression of the right-hand side does not include the gauge parameters $\phi$ explicitly but the Hamiltonian indeed depends on $\phi$ through the boundary conditions. Namely the 
boundary conditions are twisted with the angles $\phi$. Here we stress that the boundary condition in the $s$ direction becomes periodic for the special values $\phi_{s}=m \Delta \phi_{s}$ of the gauge parameter with an integer $m$ for $s=x, y$, where $\Delta \phi_{s}=2 \pi \hbar / L_{s}$ are given in (2.5). Clearly the sector of the ground state has this periodicity from Assumption 2.1 on the ground state. Therefore the ground state vectors $\tilde{\Phi}_{0, \mu}^{(N)}(\phi)$ must satisfy the relations,

$$
\tilde{\Phi}_{0, \mu}^{(N)}\left(\Delta \phi_{x}, \phi_{y}\right)=\sum_{\mu^{\prime}=1}^{q} C_{\mu, \mu^{\prime}}^{(x)}\left(\phi_{y}\right) \tilde{\Phi}_{0, \mu^{\prime}}^{(N)}\left(0, \phi_{y}\right)
$$

and

$$
\tilde{\Phi}_{0, \mu}^{(N)}\left(\phi_{x}, \Delta \phi_{y}\right)=\sum_{\mu^{\prime}=1}^{q} C_{\mu, \mu^{\prime}}^{(y)}\left(\phi_{x}\right) \tilde{\Phi}_{0, \mu^{\prime}}^{(N)}\left(\phi_{x}, 0\right),
$$

where $C^{(x)}\left(\phi_{y}\right)$ and $C^{(y)}\left(\phi_{x}\right)$ are a $q \times q$ unitary matrix as a function of $\phi_{y}$ and $\phi_{x}$, respectively.

In terms of the vectors $\tilde{\Phi}_{0, \mu}^{(N)}(\phi)$, the acceleration coefficients $\gamma_{s y}(\phi)$ of $(5.11)$ can be written as

$$
\gamma_{s y}(\phi)=\frac{e^{2}}{L_{x} L_{y}} \frac{1}{q} \sum_{\mu=1}^{q} \frac{\partial}{\partial \phi_{y}}\left\langle\tilde{\Phi}_{0, \mu}^{(N)}(\phi), v_{\mathrm{tot}, s}^{(0)}(0) \tilde{\Phi}_{0, \mu}^{(N)}(\phi)\right\rangle \quad \text { for } s=x, y,
$$

where $v_{\text {tot }, s}^{(0)}(0)=\sum_{j=1}^{N}\left[p_{s, j}+e A_{s}\left(\mathbf{r}_{j}\right)\right] / m_{e}$. Combining this with the relation (5.20), the averaged acceleration coefficients $\overline{\gamma_{s y}(\boldsymbol{\phi})}$ of (2.30) vanish as

$$
\begin{array}{rl}
\overline{\gamma_{s y}(\phi)}=\frac{1}{\Delta \phi_{x} \Delta \phi_{y}} \frac{e^{2}}{L_{x} L_{y}} \frac{1}{q} \sum_{\mu=1}^{q} \int_{0}^{\Delta \phi_{x}} & d \phi_{x}\left[\left\langle\tilde{\Phi}_{0, \mu}^{(N)}\left(\phi_{x}, \Delta \phi_{y}\right), v_{\mathrm{tot}, s}^{(0)}(0) \tilde{\Phi}_{0, \mu}^{(N)}\left(\phi_{x}, \Delta \phi_{y}\right)\right\rangle\right. \\
& \left.-\left\langle\tilde{\Phi}_{0, \mu}^{(N)}\left(\phi_{x}, 0\right), v_{\mathrm{tot}, s}^{(0)}(0) \tilde{\Phi}_{0, \mu}^{(N)}\left(\phi_{x}, 0\right)\right\rangle\right]=0
\end{array}
$$

for $s=x, y$. Here we have used the unitarity of $C^{(y)}\left(\phi_{x}\right)$.

Similarly the Hall conductance $\sigma_{x y}(\boldsymbol{\phi})$ of $(5.14)$ becomes

$$
\begin{aligned}
\sigma_{x y}(\boldsymbol{\phi}) & =-\frac{i \hbar e^{2}}{L_{x} L_{y}} \frac{1}{q} \sum_{\mu=1}^{q}\left[\frac{\partial}{\partial \phi_{x}}\left\langle\tilde{\Phi}_{0, \mu}^{(N)}(\boldsymbol{\phi}), \frac{\partial}{\partial \phi_{y}} \tilde{\Phi}_{0, \mu}^{(N)}(\boldsymbol{\phi})\right\rangle-\frac{\partial}{\partial \phi_{y}}\left\langle\tilde{\Phi}_{0, \mu}^{(N)}(\boldsymbol{\phi}), \frac{\partial}{\partial \phi_{x}} \tilde{\Phi}_{0, \mu}^{(N)}(\boldsymbol{\phi})\right\rangle\right. \\
& \left.-\frac{i}{\hbar} \frac{\partial}{\partial \phi_{x}}\left\langle\tilde{\Phi}_{0, \mu}^{(N)}(\boldsymbol{\phi}), \sum_{i=1}^{N} y_{i} \tilde{\Phi}_{0, \mu}^{(N)}(\boldsymbol{\phi})\right\rangle+\frac{i}{\hbar} \frac{\partial}{\partial \phi_{y}}\left\langle\tilde{\Phi}_{0, \mu}^{(N)}(\boldsymbol{\phi}), \sum_{j=1}^{N} x_{j} \tilde{\Phi}_{0, \mu}^{(N)}(\boldsymbol{\phi})\right\rangle\right] . \quad(5.23)
\end{aligned}
$$

By averaging over the gauge parameters $\phi, \overline{\sigma_{x y}(\phi)}$ of $(2.29)$ is written as

$$
\overline{\sigma_{x y}(\boldsymbol{\phi})}=\frac{e^{2}}{h} \frac{1}{2 \pi i} \frac{1}{q} \sum_{\mu=1}^{q} \int_{\mathcal{T}_{\mathrm{g}}} d \phi_{x} d \phi_{y}\left[\frac{\partial}{\partial \phi_{x}}\left\langle\tilde{\Phi}_{0, \mu}^{(N)}(\boldsymbol{\phi}), \frac{\partial}{\partial \phi_{y}} \tilde{\Phi}_{0, \mu}^{(N)}(\boldsymbol{\phi})\right\rangle-(x \leftrightarrow y)\right],
$$

where we have used the relations (5.19) and (5.20). Thus one get the geometrically invariant form of the Hall conductance. To see this more explicitly, we write

$$
\mathcal{A}_{\mu, \mu^{\prime}, s}(\phi)=\left\langle\tilde{\Phi}_{0, \mu}^{(N)}(\phi), \frac{\partial}{\partial \phi_{s}} \tilde{\Phi}_{0, \mu^{\prime}}^{(N)}(\phi)\right\rangle \quad \text { for } s=x, y,
$$


and

$$
\mathcal{F}(\phi)=\frac{\partial}{\partial \phi_{x}} \mathcal{A}_{y}(\phi)-\frac{\partial}{\partial \phi_{y}} \mathcal{A}_{x}(\phi)+\left[\mathcal{A}_{x}(\phi), \mathcal{A}_{y}(\phi)\right]
$$

which are, respectively, the connections and the curvature in the language of differential geometry [39]. In the language of the corresponding non-Abelian gauge theory [42], these corresponds to the gauge fields and the field strength tensor. The connections $\mathcal{A}_{s}(\phi)$ are the $q \times q$ matrix with the matrix elements $\mathcal{A}_{\mu, \mu^{\prime}, s}(\phi)$. Then the geometric invariant on the gauge torus ${ }^{12} \mathcal{T}_{\mathrm{g}}$ is given by

$$
\mathcal{I}=\frac{1}{2 \pi i} \int_{\mathcal{T}_{\mathrm{g}}} d \phi_{x} d \phi_{y} \operatorname{tr} \mathcal{F}(\phi)
$$

where tr is the trace of $q \times q$ matrix. Since $\operatorname{tr}\left[\mathcal{A}_{x}(\phi), \mathcal{A}_{y}(\phi)\right]=0$, one has

$$
\overline{\sigma_{x y}(\phi)}=\frac{e^{2}}{h} \frac{\mathcal{I}}{q}
$$

In passing, we remark that the connection $\mathcal{A}_{s}(\phi)$ is not necessarily periodic at the boundaries of the rectangular region $\mathcal{T}_{\mathrm{g}}$. If the connection $\mathcal{A}_{s}(\phi)$ satisfies the periodic boundary conditions, then the quantity $\mathcal{I}$ vanishes, i.e., the averaged Hall conductance $\overline{\sigma_{x y}(\phi)}$ becomes zero.

Following the way of computing a geometric invariant in refs. [6, 11], we shall show that the geometric invariant $\mathcal{I}$ takes an integer value, i.e., the averaged Hall conductance $\overline{\sigma_{x y}(\phi)}$ is quantized to a rational number $p / q$. For this purpose, we rewrite the averaged Hall conductance $\overline{\sigma_{x y}(\phi)}$ of $(5.24)$ as

$$
\begin{aligned}
\overline{\sigma_{x y}(\phi)}=\frac{e^{2}}{h} \frac{1}{2 \pi i} \frac{1}{q} \sum_{\mu=1}^{q} \int_{0}^{\Delta \phi_{y}} d \phi_{y} & {\left[\left\langle\tilde{\Phi}_{0, \mu}^{(N)}\left(\Delta \phi_{x}, \phi_{y}\right), \frac{\partial}{\partial \phi_{y}} \tilde{\Phi}_{0, \mu}^{(N)}\left(\Delta \phi_{x}, \phi_{y}\right)\right\rangle\right.} \\
- & \left.\left\langle\tilde{\Phi}_{0, \mu}^{(N)}\left(0, \phi_{y}\right), \frac{\partial}{\partial \phi_{y}} \tilde{\Phi}_{0, \mu}^{(N)}\left(0, \phi_{y}\right)\right\rangle\right] \\
-\frac{e^{2}}{h} \frac{1}{2 \pi i} \frac{1}{q} \sum_{\mu=1}^{q} \int_{0}^{\Delta \phi_{x}} d \phi_{x} & {\left[\left\langle\tilde{\Phi}_{0, \mu}^{(N)}\left(\phi_{x}, \Delta \phi_{y}\right), \frac{\partial}{\partial \phi_{x}} \tilde{\Phi}_{0, \mu}^{(N)}\left(\phi_{x}, \Delta \phi_{y}\right)\right\rangle\right.} \\
& \left.-\left\langle\tilde{\Phi}_{0, \mu}^{(N)}\left(\phi_{x}, 0\right), \frac{\partial}{\partial \phi_{x}} \tilde{\Phi}_{0, \mu}^{(N)}\left(\phi_{x}, 0\right)\right\rangle\right] .
\end{aligned}
$$

In order to compute this right-hand side, we introduce $q \times q$ matrices $\theta^{(x)}\left(\phi_{y}\right)$ and $\theta^{(y)}\left(\phi_{x}\right)$ as

$$
C^{(x)}\left(\phi_{y}\right)=\exp \left[i \theta^{(x)}\left(\phi_{y}\right)\right] \quad \text { and } \quad C^{(y)}\left(\phi_{x}\right)=\exp \left[i \theta^{(y)}\left(\phi_{x}\right)\right] .
$$

Using these and the relations (5.19) and (5.20) again, one has

$$
\overline{\sigma_{x y}(\boldsymbol{\phi})}=\frac{e^{2}}{h} \frac{1}{2 \pi i} \frac{1}{q} \sum_{\mu=1}^{q}\left[\int_{0}^{\Delta \phi_{y}} d \phi_{y} \frac{\partial}{\partial \phi_{y}} i \theta_{\mu, \mu}^{(x)}\left(\phi_{y}\right)-\int_{0}^{\Delta \phi_{x}} d \phi_{x} \frac{\partial}{\partial \phi_{x}} i \theta_{\mu, \mu}^{(y)}\left(\phi_{x}\right)\right]
$$

\footnotetext{
${ }^{12}$ It is necessary to impose the periodic boundary conditions on the rectangular region $\mathcal{T}_{\mathrm{g}}$ for identifying it as a torus.
} 


$$
\begin{aligned}
& =\frac{e^{2}}{2 \pi h} \frac{1}{q} \sum_{\mu=1}^{q}\left[\theta_{\mu, \mu}^{(x)}\left(\Delta \phi_{y}\right)-\theta_{\mu, \mu}^{(x)}(0)-\theta_{\mu, \mu}^{(y)}\left(\Delta \phi_{x}\right)+\theta_{\mu, \mu}^{(y)}(0)\right] \\
& =\frac{e^{2}}{2 \pi h} \frac{1}{q} \operatorname{tr}\left[\theta^{(x)}\left(\Delta \phi_{y}\right)-\theta^{(x)}(0)-\theta^{(y)}\left(\Delta \phi_{x}\right)+\theta^{(y)}(0)\right]
\end{aligned}
$$

where we have used

$$
\left\langle\tilde{\Phi}_{0, \mu}^{(N)}(\phi), \tilde{\Phi}_{0, \mu^{\prime}}^{(N)}(\phi)\right\rangle=\delta_{\mu, \mu^{\prime}}
$$

On the other hand, from the relations (5.19) and (5.20) and (5.30), one has

$$
\begin{gathered}
\tilde{\Phi}_{0}^{(N)}\left(\Delta \phi_{x}, \Delta \phi_{y}\right)=\exp \left[i \theta_{x}\left(\Delta \phi_{y}\right)\right] \tilde{\Phi}_{0}^{(N)}\left(0, \Delta \phi_{y}\right), \\
\tilde{\Phi}_{0}^{(N)}\left(\Delta \phi_{x}, 0\right)=\exp \left[i \theta_{x}(0)\right] \tilde{\Phi}_{0}^{(N)}(0,0), \\
\tilde{\Phi}_{0}^{(N)}\left(\Delta \phi_{x}, \Delta \phi_{y}\right)=\exp \left[i \theta_{y}\left(\Delta \phi_{x}\right)\right] \tilde{\Phi}_{0}^{(N)}\left(\Delta \phi_{x}, 0\right),
\end{gathered}
$$

and

$$
\tilde{\Phi}_{0}^{(N)}\left(0, \Delta \phi_{y}\right)=\exp \left[i \theta_{y}(0)\right] \tilde{\Phi}_{0}^{(N)}(0,0)
$$

where $\tilde{\Phi}_{0}^{(N)}\left(\phi_{x}, \phi_{y}\right)$ is the $q$ component vector whose $\mu$-th component is $\tilde{\Phi}_{0, \mu}^{(N)}\left(\phi_{x}, \phi_{y}\right)$. These four equations yield

$$
\exp \left[i \theta^{(x)}\left(\Delta \phi_{y}\right)\right] \exp \left[-i \theta^{(x)}(0)\right] \exp \left[-i \theta^{(y)}\left(\Delta \phi_{x}\right)\right] \exp \left[i \theta^{(y)}(0)\right]=1,
$$

where we have used the relation (5.32). Taking the determinant of both sides of this equation and using $\operatorname{det} \exp \left[i \theta^{(s)}(\cdots)\right]=\exp \left[i \operatorname{tr} \theta^{(s)}(\cdots)\right]$ for $s=x, y$, one has

$$
\operatorname{tr}\left[\theta^{(x)}\left(\Delta \phi_{y}\right)-\theta^{(x)}(0)-\theta^{(y)}\left(\Delta \phi_{x}\right)+\theta^{(y)}(0)\right]=-2 \pi p \quad \text { with an integer } p \text {. }
$$

Owing to this relation, the averaged Hall conductance $\overline{\sigma_{x y}(\phi)}$ of (5.31) must satisfy

$$
\overline{\sigma_{x y}(\phi)}=-\frac{e^{2}}{h} \frac{p}{q} \quad \text { with an integer } p \text {. }
$$

\subsection{Fractional quantization and Atiyah-Singer index theorem}

In this subsection, we will show that the integer $-p$ of the fractional quantization (5.39) of the averaged Hall conductance $\overline{\sigma_{x y}(\phi)}$ is equal to an index of a Pauli-Dirac operator $\mathcal{D}$ with the gauge field $\mathcal{A}(\phi)=\left(\mathcal{A}_{x}(\phi), \mathcal{A}_{y}(\phi)\right)$ on the gauge torus $\mathcal{T}_{\mathrm{g}}$. This is nothing but a special case of Atiyah-Singer index theorem. Namely the first Chern number $\mathcal{I}$ of (5.27) is equal to the index, ind $\mathcal{D}$, of the Pauli-Dirac operator as we will see in Theorem 5.5 below. The corresponding system described by the Hamiltonian $H=-\mathcal{D}^{2}$ is equivalent to a single electron system with spin-1/2 and with $q$ flavors in the gauge field $\mathcal{A}(\boldsymbol{\phi})$ on the torus $\mathcal{T}_{\mathrm{g}}$, and the index, $-p=$ ind $\mathcal{D}$, is equal to the difference of the degeneracy between the up-spin and the down-spin ground states. Thus the integer $p$ of the fractionally quantized Hall conductance of the interacting electrons is closely related to the ground state property of the single electron coupled to the gauge field $\mathcal{A}(\phi)$ determined by the ground state of the original interacting electron system. 
Throughout the present subsection, for simplicity, we will write $\tilde{\Phi}_{\mu}(\phi)=\tilde{\Phi}_{0, \mu}^{(N)}(\phi)$, $\mu=1,2, \ldots, q$, for the ground state wavefunctions, by dropping the subscript 0 and the superscript $(N)$. Then the matrix elements of the gauge field $\mathcal{A}(\phi)=\left(\mathcal{A}_{x}(\phi), \mathcal{A}_{y}(\phi)\right)$ are written in terms of $\tilde{\Phi}_{\mu}(\boldsymbol{\phi})$ as

$$
\mathcal{A}_{\mu, \nu, s}(\phi)=\left\langle\tilde{\Phi}_{\mu}(\phi), \partial_{s} \tilde{\Phi}_{\nu}(\phi)\right\rangle \quad \text { for } s=x, y
$$

where we have written

$$
\partial_{s}=\frac{\partial}{\partial \phi_{s}} \quad \text { for } s=x, y
$$

We define gauge transformations as

$$
\mathcal{G}_{\mu, \nu}^{(x)}\left(\phi_{x}, \phi_{y}\right):=\left\langle\tilde{\Phi}_{\mu}\left(\phi_{x}+\Delta \phi_{x}, \phi_{y}\right), \tilde{\Phi}_{\nu}\left(\phi_{x}, \phi_{y}\right)\right\rangle \text { for small } \phi_{x}
$$

and

$$
\mathcal{G}_{\mu, \nu}^{(y)}\left(\phi_{x}, \phi_{y}\right):=\left\langle\tilde{\Phi}_{\mu}\left(\phi_{x}, \phi_{y}+\Delta \phi_{y}\right), \tilde{\Phi}_{\nu}\left(\phi_{x}, \phi_{y}\right)\right\rangle \text { for small } \phi_{y}
$$

These are $q \times q$ unitary matrices. Actually,

$$
\begin{aligned}
\sum_{\alpha=1}^{q} \mathcal{G}_{\mu, \alpha}^{(x)}(\phi) \mathcal{G}_{\nu, \alpha}^{(x)}(\boldsymbol{\phi})^{*} & =\sum_{\alpha=1}^{q}\left\langle\tilde{\Phi}_{\mu}\left(\phi_{x}+\Delta \phi_{x}, \phi_{y}\right), \tilde{\Phi}_{\alpha}(\phi)\right\rangle\left\langle\tilde{\Phi}_{\alpha}(\phi), \tilde{\Phi}_{\nu}\left(\phi_{x}+\Delta \phi_{x}, \phi_{y}\right)\right\rangle \\
& =\left\langle\tilde{\Phi}_{\mu}\left(\phi_{x}+\Delta \phi_{x}, \phi_{y}\right), \tilde{\Phi}_{\nu}\left(\phi_{x}+\Delta \phi_{x}, \phi_{y}\right)\right\rangle=\delta_{\mu, \nu}
\end{aligned}
$$

Similarly, one has

$$
\sum_{\alpha=1}^{q} \mathcal{G}_{\mu, \alpha}^{(y)}(\phi) \mathcal{G}_{\nu, \alpha}^{(y)}(\phi)^{*}=\delta_{\mu, \nu} .
$$

Lemma 5.2 The gauge field $\mathcal{A}(\phi)=\left(\mathcal{A}_{x}(\phi), \mathcal{A}_{y}(\phi)\right)$ satisfies

$$
\mathcal{A}_{s}\left(\phi_{x}+\Delta \phi_{x}, \phi_{y}\right)=\mathcal{G}^{(x)}(\phi) \mathcal{A}_{s}(\phi) \mathcal{G}^{(x)}(\phi)^{*}+\mathcal{G}^{(x)}(\phi) \partial_{s} \mathcal{G}^{(x)}(\phi)^{*} \quad \text { for small } \phi_{x}
$$

and

$$
\mathcal{A}_{s}\left(\phi_{x}, \phi_{y}+\Delta \phi_{y}\right)=\mathcal{G}^{(y)}(\phi) \mathcal{A}_{s}(\phi) \mathcal{G}^{(y)}(\phi)^{*}+\mathcal{G}^{(y)}(\phi) \partial_{s} \mathcal{G}^{(y)}(\phi)^{*} \quad \text { for small } \phi_{y}
$$

Proof: From the definitions (5.40), (5.42) and (5.43) for the gauge field $\mathcal{A}(\phi)$ and the gauge transformations $\mathcal{G}^{(s)}(\phi)$ for $s=x, y$, we have

$$
\begin{aligned}
& \mathcal{A}_{\mu, \nu, s}\left(\phi_{x}+\Delta \phi_{x}, \phi_{y}\right) \\
= & \left\langle\tilde{\Phi}_{\mu}\left(\phi_{x}+\Delta \phi_{x}, \phi_{y}\right), \partial_{s} \tilde{\Phi}_{\nu}\left(\phi_{x}+\Delta \phi_{x}, \phi_{y}\right)\right\rangle \\
= & \sum_{\alpha, \beta=1}^{q}\left\langle\tilde{\Phi}_{\mu}\left(\phi_{x}+\Delta \phi_{x}, \phi_{y}\right), \tilde{\Phi}_{\alpha}(\phi)\right\rangle\left\langle\tilde{\Phi}_{\alpha}(\phi), \partial_{s} \tilde{\Phi}_{\beta}(\phi)\right\rangle\left\langle\tilde{\Phi}_{\beta}(\phi), \tilde{\Phi}_{\nu}\left(\phi_{x}+\Delta \phi_{x}, \phi_{y}\right)\right\rangle \\
= & \sum_{\alpha, \beta}\left\{\mathcal{G}_{\mu, \alpha}^{(x)}(\phi) \mathcal{A}_{\alpha, \beta, s}(\phi) \mathcal{G}_{\nu, \beta}^{(x)}(\phi)^{*}+\mathcal{G}_{\mu, \alpha}^{(x)}(\phi) \delta_{\alpha, \beta} \partial_{s} \mathcal{G}_{\nu, \beta}^{(x)}(\phi)^{*}\right\} \\
= & \left(\mathcal{G}^{(x)}(\boldsymbol{\phi}) \mathcal{A}_{s}(\boldsymbol{\phi}) \mathcal{G}^{(x)}(\boldsymbol{\phi})^{*}\right)_{\mu, \nu}+\left(\mathcal{G}^{(x)}(\phi) \partial_{s} \mathcal{G}^{(x)}(\phi)^{*}\right)_{\mu, \nu} .
\end{aligned}
$$


In the same way, the other relation (5.47) can be obtained.

We define covariant derivatives $\nabla_{s}$ as

$$
\nabla_{s}:=\partial_{s}+\mathcal{A}_{s}(\phi) \text { for } s=x, y .
$$

These covariant derivatives $\nabla_{s}$ act on a vector field $f(\phi)=\left(f_{1}(\phi), f_{2}(\phi), \cdots, f_{q}(\phi)\right)$ on the torus $\mathcal{T}_{\mathrm{g}}$. We require that such a vector field $f(\phi)$ is transformed as

$$
f_{\mu}\left(\phi_{x}+\Delta \phi_{x}, \phi_{y}\right)=\sum_{\alpha=1}^{q} \mathcal{G}_{\mu, \alpha}^{(x)}(\phi) f_{\alpha}(\phi) \text { for small } \phi_{x}
$$

and

$$
f_{\mu}\left(\phi_{x}, \phi_{y}+\Delta \phi_{y}\right)=\sum_{\alpha=1}^{q} \mathcal{G}_{\mu, \alpha}^{(y)}(\phi) f_{\alpha}(\phi) \text { for small } \phi_{y},
$$

by the gauge transformation $\mathcal{G}^{(s)}(\phi)$. Then one has

Lemma 5.3 Assume the vector field $f(\phi)$ is continuously differentiable with respect to the gauge parameters $\phi=\left(\phi_{x}, \phi_{y}\right)$. Then $f(\phi)$ satisfies

$$
\left(\nabla_{s} f\right)\left(\phi_{x}+\Delta \phi_{x}, \phi_{y}\right)=\mathcal{G}^{(x)}(\phi)\left(\nabla_{s} f\right)\left(\phi_{x}, \phi_{y}\right) \text { for small } \phi_{x},
$$

and

$$
\left(\nabla_{s} f\right)\left(\phi_{x}, \phi_{y}+\Delta \phi_{y}\right)=\mathcal{G}^{(y)}(\phi)\left(\nabla_{s} f\right)\left(\phi_{x}, \phi_{y}\right) \text { for small } \phi_{y} .
$$

Proof: From the transformation (5.50) for the vector field $f(\phi)$, one has

$$
\partial_{s} f_{\mu}\left(\phi_{x}+\Delta \phi_{x}, \phi_{y}\right)=\sum_{\alpha=1}^{q}\left[\partial_{s} \mathcal{G}_{\mu, \alpha}^{(x)}(\phi) \cdot f_{\alpha}(\phi)+\mathcal{G}_{\mu, \alpha}^{(x)}(\phi) \partial_{s} f_{\alpha}(\phi)\right]
$$

for small $\phi_{x}$. From the transformation (5.50) and Lemma 5.2, one has

$$
\begin{aligned}
& \sum_{\alpha=1}^{q} \mathcal{A}_{\mu, \alpha, s}\left(\phi_{x}+\Delta \phi_{x}, \phi_{y}\right) f_{\alpha}\left(\phi_{x}+\Delta \phi_{x}, \phi_{y}\right) \\
= & \left(\mathcal{G}^{(x)}(\boldsymbol{\phi}) \mathcal{A}_{s}(\phi) f(\phi)\right)_{\mu}+\sum_{\alpha=1}^{q}\left(\mathcal{G}^{(x)}(\phi) \partial_{s} \mathcal{G}^{(x)}(\phi)^{*}\right)_{\mu, \alpha}\left(\mathcal{G}^{(x)}(\phi) f(\phi)\right)_{\alpha} \\
= & \left(\mathcal{G}^{(x)}(\phi) \mathcal{A}_{s}(\phi) f(\phi)\right)_{\mu}-\left(\partial_{s} \mathcal{G}^{(x)}(\phi) \cdot f(\phi)\right)_{\mu},
\end{aligned}
$$

where we have used the identity,

$$
\mathcal{G}^{(s)}(\phi) \partial_{t} \mathcal{G}^{(s)}(\phi)^{*}+\partial_{t} \mathcal{G}^{(s)}(\phi) \cdot \mathcal{G}^{(s)}(\phi)^{*}=0 \quad \text { for } \quad s, t=x, y,
$$

for getting the second equality. Combining (5.54) and (5.55), the relation (5.52) is derived. The other relation is also derived in the same way.

We introduce the Pauli-Dirac operator $\mathcal{D}$ as

$$
\mathcal{D}=\sigma_{x} \nabla_{x}+\sigma_{y} \nabla_{y},
$$


where $\sigma_{x}$ and $\sigma_{y}$ are the Pauli matrices given by

$$
\sigma_{x}=\left(\begin{array}{ll}
0 & 1 \\
1 & 0
\end{array}\right) \quad \text { and } \quad \sigma_{y}=\left(\begin{array}{rr}
0 & -i \\
i & 0
\end{array}\right) .
$$

Clearly the Pauli-Dirac operator $\mathcal{D}$ is written as

$$
\mathcal{D}=\left(\begin{array}{cc}
0 & \mathcal{D}^{-} \\
\mathcal{D}^{+} & 0
\end{array}\right),
$$

where

$$
\mathcal{D}^{+}=\nabla_{x}+i \nabla_{y} \quad \text { and } \quad \mathcal{D}^{-}=\nabla_{x}-i \nabla_{y} .
$$

The Hamiltonian of the corresponding system is given by

$$
H:=-\mathcal{D}^{2}=\left(\begin{array}{cc}
-\mathcal{D}^{-} \mathcal{D}^{+} & 0 \\
0 & -\mathcal{D}^{+} \mathcal{D}^{-}
\end{array}\right) .
$$

Note that

$$
\begin{aligned}
\mathcal{D}^{-} \mathcal{D}^{+} & =\left(\nabla_{x}-i \nabla_{y}\right)\left(\nabla_{x}+i \nabla_{y}\right) \\
& =\nabla_{x}^{2}+\nabla_{y}^{2}+i \nabla_{x} \nabla_{y}-i \nabla_{y} \nabla_{x} \\
& =\nabla_{x}^{2}+\nabla_{y}^{2}+i\left[\nabla_{x}, \nabla_{y}\right] .
\end{aligned}
$$

Since the commutator in the last line is equal to the curvature $\mathcal{F}$ as

$$
\left[\nabla_{x}, \nabla_{y}\right]=\partial_{x} \mathcal{A}_{y}-\partial_{y} \mathcal{A}_{x}+\left[\mathcal{A}_{x}, \mathcal{A}_{y}\right]=: \mathcal{F},
$$

one has

$$
\mathcal{D}^{-} \mathcal{D}^{+}=\triangle+i \mathcal{F}
$$

with the Laplacian,

$$
\triangle=\nabla_{x}^{2}+\nabla_{y}^{2}=\left(\partial_{x}+\mathcal{A}_{x}\right)^{2}+\left(\partial_{y}+\mathcal{A}_{y}\right)^{2} .
$$

In the same way, one has

$$
\mathcal{D}^{+} \mathcal{D}^{-}=\triangle-i \mathcal{F} .
$$

Therefore the Hamiltonian $H$ becomes

$$
H=-\triangle-i \mathcal{F} \sigma_{z} \quad \text { with } \quad \sigma_{z}:=\left(\begin{array}{cc}
1 & 0 \\
0 & -1
\end{array}\right) .
$$

Namely the system is equivalent to the single electron with spin- $1 / 2$ and $q$ flavors in the "magnetic field" $\mathcal{F}$ in two dimensions. Here we stress that the number $q$ of the flavors is equal to the degeneracy of the ground state of the original interacting electron system.

We define the index of the Pauli-Dirac operator $\mathcal{D}$ as

$$
\text { ind } \mathcal{D}:=\operatorname{dim} \operatorname{ker} \mathcal{D}^{+}-\operatorname{dim} \operatorname{ker} \mathcal{D}^{-} \text {. }
$$

In the standard way, one has 
Lemma 5.4 For any positive constant $\beta$, the following equality is valid:

$$
\text { ind } \mathcal{D}=\operatorname{Tr} \sigma_{z} e^{-\beta H} \text {. }
$$

Proof: Let $\psi$ be an eigenvector of the operator $\mathcal{D}^{-} \mathcal{D}^{+}$with the eigenvalue $\lambda \neq 0$, i.e.,

$$
\mathcal{D}^{-} \mathcal{D}^{+} \psi=\lambda \psi
$$

Then the vector $\mathcal{D}^{+} \psi$ is the eigenvector of the operator $\mathcal{D}^{+} \mathcal{D}^{-}$with the same eigenvalue $\lambda$. Actually, one has

$$
\left\|\mathcal{D}^{+} \psi\right\|^{2}=\left\langle\mathcal{D}^{+} \psi, \mathcal{D}^{+} \psi\right\rangle=-\left\langle\psi, \mathcal{D}^{-} \mathcal{D}^{+} \psi\right\rangle=-\lambda\|\psi\|^{2} \neq 0
$$

and

$$
\mathcal{D}^{+} \mathcal{D}^{-}\left(\mathcal{D}^{+} \psi\right)=\mathcal{D}^{+}\left(\mathcal{D}^{-} \mathcal{D}^{+} \psi\right)=\lambda \mathcal{D}^{+} \psi
$$

Let $\psi_{1}, \psi_{2}$ be eigenvectors of $\mathcal{D}^{-} \mathcal{D}^{+}$with the eigenvalue $\lambda$, and assume the two vectors are orthogonal to each other. Then

$$
\left\langle\mathcal{D}^{+} \psi_{1}, \mathcal{D}^{+} \psi_{2}\right\rangle=-\left\langle\psi_{1}, \mathcal{D}^{-} \mathcal{D}^{+} \psi_{2}\right\rangle=-\lambda\left\langle\psi_{1}, \psi_{2}\right\rangle=0
$$

We denote by $d^{+}(\lambda)$ the dimension of the eigenspace of the operator $\mathcal{D}^{-} \mathcal{D}^{+}$with the eigenvalue $\lambda$, and by $d^{-}(\lambda)$ the dimension of the eigenspace of $\mathcal{D}^{+} \mathcal{D}^{-}$with $\lambda$. From the above observations, one has $d^{+}(\lambda) \leq d^{-}(\lambda)$ for $\lambda \neq 0$. Further $d^{-}(\lambda) \leq d^{+}(\lambda)$ for $\lambda \neq 0$ in the same way. Combining these two inequalities, one has $d^{+}(\lambda)=d^{-}(\lambda)$ for $\lambda \neq 0$. From this fact, one gets

$$
\operatorname{Tr} \sigma_{z} e^{-\beta H}=\operatorname{Tr} e^{\beta \mathcal{D}^{-} \mathcal{D}^{+}}-\operatorname{Tr} e^{\beta \mathcal{D}^{+} \mathcal{D}^{-}}=\operatorname{dim} \operatorname{ker} \mathcal{D}^{-} \mathcal{D}^{+}-\operatorname{dim} \operatorname{ker} \mathcal{D}^{+} \mathcal{D}^{-}
$$

This right-hand side is equal to the index of the operator $\mathcal{D}$ by the definition (5.68).

The following theorem is a special case of the Atiyah-Singer index theorem [40, 41]:

\section{Theorem 5.5}

$$
\text { ind } \mathcal{D}=\frac{1}{2 \pi i} \int_{\mathcal{T}_{\mathrm{g}}} d \phi_{x} d \phi_{y} \operatorname{tr} \mathcal{F}
$$

Proof: We shall prove this statement along the same line as in ref. [49]. First let us introduce the complete system of the orthonormal functions as

$$
\psi\left(k_{x}, k_{y}\right):=\frac{1}{\sqrt{\Delta \phi_{x} \Delta \phi_{y}}} \exp \left[i\left(k_{x} \phi_{x}+k_{y} \phi_{y}\right)\right] \quad \text { with } k_{x}=\frac{2 \pi n_{x}}{\Delta \phi_{x}}, k_{y}=\frac{2 \pi n_{y}}{\Delta \phi_{y}}
$$

with $n_{x}, n_{y} \in \mathbf{Z}$. Using this system of the functions, one has

$$
\begin{aligned}
\operatorname{Tr} e^{\beta(\triangle \pm i \mathcal{F})} & =\sum_{k_{x}, k_{y}} \operatorname{tr}\left\langle\psi\left(k_{x}, k_{y}\right), e^{\beta(\Delta \pm i \mathcal{F})} \psi\left(k_{x}, k_{y}\right)\right\rangle \\
& =\frac{1}{\Delta \phi_{x} \Delta \phi_{y}} \sum_{k_{x}, k_{y}} \operatorname{tr} \int_{\mathcal{T}_{\mathrm{g}}} d \phi_{x} d \phi_{y} \exp [\beta(\triangle(\mathbf{k}) \pm i \mathcal{F})]
\end{aligned}
$$


Revisiting the Charge Transport in Quantum Hall Systems

where we have written

$$
\triangle(\mathbf{k})=\left(\nabla_{x}+i k_{x}\right)^{2}+\left(\nabla_{y}+i k_{y}\right)^{2}
$$

and have used

$$
e^{-i k_{s} \phi_{s}} \partial_{s} e^{i k_{s} \phi_{s}}=\partial_{s}+i k_{s} \quad \text { for } s=x, y .
$$

Moreover, by using DuHamel's formula

$$
e^{-t(X+Y)}=e^{-t X}+\int_{0}^{t} d s e^{-(t-s) X} Y e^{-s(X+Y)},
$$

one has

$$
\begin{aligned}
& \operatorname{Tr} e^{\beta(\Delta+i \mathcal{F})}-\operatorname{Tr} e^{\beta(\Delta-i \mathcal{F})} \\
= & \frac{\beta}{i \Delta \phi_{x} \Delta \phi_{y}} \sum_{k_{x}, k_{y}} \operatorname{tr} \int_{\mathcal{T}_{\mathfrak{g}}} d \phi_{x} d \phi_{y} \frac{1}{\beta} \int_{0}^{\beta} d \beta^{\prime} e^{\left(\beta-\beta^{\prime}\right) \Delta(\mathbf{k})} \mathcal{F}\left[e^{\beta^{\prime}(\Delta(\mathbf{k})+i \mathcal{F})}+e^{\beta^{\prime}(\Delta(\mathbf{k})-i \mathcal{F})}\right] \\
= & \frac{\beta}{i \Delta \phi_{x} \Delta \phi_{y}} \sum_{k_{x}, k_{y}} \operatorname{tr} \int_{\mathcal{T}_{\mathrm{g}}} d \phi_{x} d \phi_{y} \int_{0}^{1} d s e^{(1-s) \beta \Delta(\mathbf{k})} \mathcal{F}\left[e^{s \beta(\Delta(\mathbf{k})+i \mathcal{F})}+e^{s \beta(\Delta(\mathbf{k})-i \mathcal{F})}\right] \\
= & \frac{\beta}{i \Delta \phi_{x} \Delta \phi_{y}} \sum_{\tilde{k}_{x}, \tilde{k}_{y}} \operatorname{tr} \int_{\mathcal{T}_{\mathrm{g}}} d \phi_{x} d \phi_{y} \int_{0}^{1} d s e^{(1-s) \tilde{\Delta}(\tilde{\mathbf{k}})} \mathcal{F}\left[e^{s(\tilde{\Delta}(\tilde{\mathbf{k}})+i \beta \mathcal{F})}+e^{s(\tilde{\Delta}(\tilde{\mathbf{k}})-i \beta \mathcal{F})}\right],(5
\end{aligned}
$$

where we have introduced the variable $s$ as $\beta^{\prime}=\beta s$ with $0 \leq s \leq 1$, and we have written

$$
\tilde{k}_{x}=\sqrt{\beta} k_{x} \text { and } \quad \tilde{k}_{y}=\sqrt{\beta} k_{y}
$$

and

$$
\tilde{\triangle}(\tilde{\mathbf{k}})=\left(\sqrt{\beta} \nabla_{x}+i \tilde{k}_{x}\right)^{2}+\left(\sqrt{\beta} \nabla_{y}+i \tilde{k}_{y}\right)^{2} .
$$

Here, since the sum of the operators

$$
\frac{\beta}{\Delta \phi_{x} \Delta \phi_{y}} \sum_{\tilde{k}_{x}, \tilde{k}_{y}} \exp [\tilde{\triangle}(\tilde{\mathbf{k}})]
$$

is uniformly bounded in $\beta$, the right-hand side of (5.81) converges to

$$
\frac{1}{(2 \pi)^{2} i} \int d \tilde{k}_{x} d \tilde{k}_{y} \operatorname{tr} \int_{\mathcal{T}_{\mathrm{g}}} d \phi_{x} d \phi_{y} \exp \left[-\left(\tilde{k}_{x}^{2}+\tilde{k}_{y}^{2}\right)\right] \times 2 \mathcal{F}=\frac{1}{2 \pi i} \int_{\mathcal{T}_{\mathrm{g}}} d \phi_{x} d \phi_{y} \operatorname{tr} \mathcal{F}
$$

in the limit $\beta \downarrow 0$. Combining this observation, (5.64), (5.66), Lemma 5.4 and (5.74), one obtains the desired relation,

$$
\text { ind } \mathcal{D}=\operatorname{Tr} e^{\beta(\triangle+i \mathcal{F})}-\operatorname{Tr} e^{\beta(\Delta-i \mathcal{F})}=\frac{1}{2 \pi i} \int_{\mathcal{T}_{\mathrm{g}}} d \phi_{x} d \phi_{y} \operatorname{tr} \mathcal{F}
$$




\section{The non-interacting case}

As a demonstration, we first treat the simplest model of the quantum Hall system, and determine the explicit forms of the function $\theta^{(s)}(\phi), s=x, y$, introduced in Section 5.1. As a result, the well-known integral quantization of the Hall conductance is obtained. In the next subsection, we will treat the general non-interacting case, and give the proof of Theorem 2.3.

\subsection{The single electron Landau Hamiltonian}

The single electron Hamiltonian in two dimensions with the uniform magnetic field only is given by

$$
\mathcal{H}_{0}(\boldsymbol{\phi})=\frac{1}{2 m_{e}}\left[\left(p_{x}-e B y+\phi_{x}\right)^{2}+\left(p_{y}+\phi_{y}\right)^{2}\right]
$$

with the gauge parameters $\boldsymbol{\phi}=\left(\phi_{x}, \phi_{y}\right) \in \mathbf{R}^{2}$. The eigenvectors on $\mathbf{R}^{2}$ are given by

$$
\varphi_{n, k}(x, y ; \phi)=e^{i k x} \exp \left[-\frac{i}{\hbar} \phi_{y} y\right] v_{n}\left(y-y\left(k, \phi_{x}\right)\right),
$$

where $k$ is the real wavenumber in the $x$ direction, and

$$
v_{n, k}\left(y ; \phi_{x}\right):=v_{n}\left(y-y\left(k, \phi_{x}\right)\right):=N_{n} \exp \left[-\left(y-y\left(k, \phi_{x}\right)\right)^{2} /\left(2 \ell_{B}^{2}\right)\right] \wp_{n}\left[\left(y-y\left(k, \phi_{x}\right)\right) / \ell_{B}\right]
$$

with

$$
y\left(k, \phi_{x}\right):=\frac{1}{e B}\left(\hbar k+\phi_{x}\right) .
$$

Here $\wp_{n}$ is the $n$-th Hermite polynomial, and the normalization constant $N_{n}$ is taken to satisfy

$$
\int_{-\infty}^{+\infty} d y\left|v_{n, k}\left(y ; \phi_{x}\right)\right|^{2}=1
$$

Now consider the $L_{x} \times L_{y}$ rectangular box $\mathcal{T}=\left[-L_{x} / 2, L_{x} / 2\right] \times\left[-L_{y} / 2, L_{y} / 2\right]$ satisfying $L_{x} L_{y}=2 \pi M \ell_{B}^{2}$ with a sufficiently large positive and even integer $M$. We impose the periodic boundary conditions

$$
\varphi(x, y ; \boldsymbol{\phi})=t^{(x)}\left(L_{x}\right) \varphi(x, y ; \boldsymbol{\phi}), \quad \varphi(x, y ; \boldsymbol{\phi})=t^{(y)}\left(L_{y}\right) \varphi(x, y ; \boldsymbol{\phi})
$$

for the wavefunctions $\varphi$. Then the complete system of the eigenvectors [31, 32] of the Hamiltonian satisfying the periodic boundary conditions is given by

$$
\varphi_{n, k}^{\mathrm{P}}(x, y ; \phi)=L_{x}^{-1 / 2} \sum_{\ell=-\infty}^{+\infty} e^{i(k+\ell K) x} \exp \left[-\frac{i}{\hbar} \phi_{y}\left(y-\ell L_{y}\right)\right] v_{n}\left(y-y\left(k, \phi_{x}\right)-\ell L_{y}\right)
$$

for $k=2 \pi m / L_{x}$ with $m=-M / 2+1, \ldots, M / 2-1, M / 2$, and with $K=L_{y} / \ell_{B}^{2}$. The energy eigenvalue is given by $(n+1 / 2) \hbar \omega_{c}$ for $n=0,1,2 \ldots$. By the gauge transformation

$$
G(x, y ; \phi)=\exp \left[\frac{i}{\hbar}\left(x \phi_{x}+y \phi_{y}\right)\right]
$$


corresponding to the transformation (5.17) of $N$ body, the eigenvectors are transformed as

$$
\begin{aligned}
\tilde{\varphi}_{n, k}^{\mathrm{P}}(x, y ; \phi) & =G(x, y ; \boldsymbol{\phi}) \varphi_{n, k}^{\mathrm{P}}(x, y ; \boldsymbol{\phi}) \\
& =L_{x}^{-1 / 2} \sum_{\ell=-\infty}^{+\infty} e^{i(k+\ell K) x} \exp \left[\frac{i}{\hbar}\left(x \phi_{x}+\ell L_{y} \phi_{y}\right)\right] v_{n}\left(y-y\left(k, \phi_{x}\right)-\ell L_{y}\right) .
\end{aligned}
$$

In order to determine the explicit forms of the phases $\theta^{(x)}$ and $\theta^{(y)}$ in (5.30), we first show that

$$
\begin{aligned}
& \tilde{\varphi}_{n, k}^{\mathrm{P}}\left(x, y ; \Delta \phi_{x}, \phi_{y}\right)=\tilde{\varphi}_{n, k+2 \pi / L_{x}}^{\mathrm{P}}\left(x, y ; 0, \phi_{y}\right) \text { for } k \neq k_{\max }, \\
& \tilde{\varphi}_{n, k_{\max }}^{\mathrm{P}}\left(x, y ; \Delta \phi_{x}, \phi_{y}\right)=\exp \left[-\frac{i}{\hbar} L_{y} \phi_{y}\right] \tilde{\varphi}_{n, k_{\min }}^{\mathrm{P}}\left(x, y ; 0, \phi_{y}\right),
\end{aligned}
$$

and

$$
\tilde{\varphi}_{n, k}^{\mathrm{P}}\left(x, y ; \phi_{x}, \Delta \phi_{y}\right)=\tilde{\varphi}_{n, k}^{\mathrm{P}}\left(x, y ; \phi_{x}, 0\right) \quad \text { for any } k,
$$

where $k_{\max }:=\pi M / L_{x}$ and $k_{\min }:=2 \pi(-M / 2+1) / L_{x}$. This last relation (6.12) is immediately obtained from the expression (6.9) of $\tilde{\varphi}_{n, k}^{\mathrm{P}}$. By the definition (6.4), one has

$$
y\left(k, \Delta \phi_{x}\right)=y\left(k+2 \pi / L_{x}, 0\right) \text { for } k \neq k_{\max } .
$$

Combining this with the expression (6.9), one obtains the first relation (6.10). For the largest wavenumber $k_{\max }=\pi M / L_{x}$, one has

$$
k_{\max }+\frac{2 \pi}{L_{x}}=k_{\min }+K
$$

where we have used the relations $K=L_{y} / \ell_{B}^{2}$ and $L_{x} L_{y}=2 \pi M \ell_{B}^{2}$. Substituting this into (6.4), one gets

$$
y\left(k_{\max }, \Delta \phi_{x}\right)=y\left(k_{\min }, 0\right)+L_{y} .
$$

Combining these with the expression (6.9), the second relation (6.11) is obtained.

Let us consider the ground state $\tilde{\Phi}_{0}^{(N)}(\phi)$ with $N=\ell M$ electrons, where $\ell$ is a positive integer and $M$ is the number of the states in a single Landau level. Namely all the states in the lowest $\ell$ Landau levels are occupied with the $\ell M$ electrons and the rest of the higher Landau levels are all empty. Clearly the ground state is unique, i.e., $q=1$, and the energy gap $\hbar \omega_{c}$ appears above the ground state. Combining (5.19), (5.30) and the above results, (6.10) and (6.11), one gets

$$
\theta^{(x)}\left(\phi_{y}\right)=-\frac{L_{y}}{\hbar} \phi_{y} \ell+\delta^{(x)}
$$

for the $N=\ell M$ electrons ground state $\tilde{\Phi}_{0}^{(N)}(\phi)$. Here $\delta^{(x)}$ is a real constant which is independent of $\boldsymbol{\phi}$. Combining (5.20) and (5.30) and (6.12), one obtains

$$
\theta^{(y)}\left(\phi_{x}\right)=0 \text { for all } \phi_{x} .
$$

From (5.31), (6.16) and (6.17), the averaged Hall conductance is given by

$$
\overline{\sigma_{x y}(\phi)}=-\frac{e^{2}}{h} \ell
$$

Since the present system is translationally invariant, the non-averaged Hall conductance is also quantized to the same integer as we already obtained the more general result (4.2). 


\subsection{The general electron gases}

Consider the general non-interacting case, i.e., $W^{(2)}=0$ in the Hamiltonian (5.1). The corresponding single electron Hamiltonian is given by

$$
\mathcal{H}(\boldsymbol{\phi})=\frac{1}{2 m_{e}}[\mathbf{p}+e \mathbf{A}(\mathbf{r})+\boldsymbol{\phi}]^{2}+W(\mathbf{r})
$$

with the vector potential $\mathbf{A}=\mathbf{A}_{0}+\mathbf{A}_{\mathrm{P}}$ and with the periodic boundary conditions (6.6). Consider the ground state $\Phi_{0}^{(N)}(\phi)$ with $N=\ell M$ electrons, where $\ell$ is a positive integer and $M$ is the number of the states in a single Landau level. This is the same situation as in the preceding subsection except for the potentials. The aim of this subsection is to prove all the statements of Theorem 2.3.

First of all, we show that the gap condition (2.17) is valid if the vector potential $\mathbf{A}_{\mathrm{P}}$ and the electrostatic potential $W$ satisfy the condition (2.36) in Theorem 2.3. For this purpose, we first rewrite the single electron Hamiltonian (6.19) as

$$
\begin{aligned}
\mathcal{H}(\boldsymbol{\phi}) & =\mathcal{H}_{0}(\boldsymbol{\phi})+\frac{e}{2 m_{e}} \mathbf{A}_{\mathrm{P}}(\mathbf{r}) \cdot\left[\mathbf{p}+e \mathbf{A}_{0}(\mathbf{r})+\phi\right]+\frac{e}{2 m_{e}}\left[\mathbf{p}+e \mathbf{A}_{0}(\mathbf{r})+\boldsymbol{\phi}\right] \cdot \mathbf{A}_{\mathrm{P}}(\mathbf{r}) \\
& +\frac{e^{2}}{2 m_{e}}\left|\mathbf{A}_{\mathrm{P}}(\mathbf{r})\right|^{2}+W(\mathbf{r})
\end{aligned}
$$

where $\mathcal{H}_{0}(\phi)$ is given by (6.1). Using the Schwarz inequality, one has

$$
\begin{aligned}
\left|\left(\psi, \frac{e}{2 m_{e}} \mathbf{A}_{\mathbf{P}} \cdot\left(\mathbf{p}+e \mathbf{A}_{0}\right) \psi\right)\right| & \leq \frac{e}{2 m_{e}} \sqrt{\left(\psi,\left|\mathbf{A}_{\mathrm{P}}\right|^{2} \psi\right)} \sqrt{\left(\psi,\left(\mathbf{p}+e \mathbf{A}_{0}\right)^{2} \psi\right)} \\
& \leq \frac{e}{2 m_{e}}\left\|\mathbf{A}_{\mathrm{P}}\right\|_{\infty} \sqrt{\left(\psi,\left(\mathbf{p}+e \mathbf{A}_{0}\right)^{2} \psi\right)}
\end{aligned}
$$

for the vector $\psi$ in the domain of the Hamiltonian. From this inequality, the energy expectation can be evaluated as

$$
(\psi, \mathcal{H}(\boldsymbol{\phi}) \psi) \leq\left(\psi, \mathcal{H}_{0}(\boldsymbol{\phi}) \psi\right)+\frac{\sqrt{2} e}{\sqrt{m_{e}}}\left\|\left|\mathbf{A}_{\mathrm{P}}\right|\right\|_{\infty} \sqrt{\left(\psi, \mathcal{H}_{0}(\boldsymbol{\phi}) \psi\right)}+\frac{e^{2}}{2 m_{e}}\left\|\left|\mathbf{A}_{\mathrm{P}}\right|\right\|_{\infty}^{2}+\left\|W^{+}\right\|_{\infty}
$$

and

$$
(\psi, \mathcal{H}(\phi) \psi) \geq\left(\psi, \mathcal{H}_{0}(\phi) \psi\right)-\frac{\sqrt{2} e}{\sqrt{m_{e}}}\left\|\mid \mathbf{A}_{\mathrm{P}}\right\|_{\infty} \sqrt{\left(\psi, \mathcal{H}_{0}(\phi) \psi\right)}-\left\|W^{-}\right\|_{\infty}
$$

Let us denote by $\mathcal{E}_{n,+}^{\text {edge }}$ and $\mathcal{E}_{n,-}^{\text {edge }}$ the upper and lower edges of the Landau band with the index $n$, respectively. From the standard argument about the min-max principle, ${ }^{13}$ one has

$$
\mathcal{E}_{n,+}^{\text {edge }} \leq(n+1 / 2) \hbar \omega_{c}+\frac{\sqrt{2} e}{\sqrt{m_{e}}}\left\|\left|\mathbf{A}_{\mathrm{P}}\right|\right\|_{\infty} \sqrt{(n+1 / 2) \hbar \omega_{c}}+\frac{e^{2}}{2 m_{e}}\left\|\left|\mathbf{A}_{\mathrm{P}}\right|\right\|_{\infty}^{2}+\left\|W^{+}\right\|_{\infty}
$$

\footnotetext{
${ }^{13}$ See, for example, Section XIII.1 of the book [50] by M. Reed and B. Simon.
} 
for $n=0,1,2, \ldots$. For the lower edge, we assume

$$
\frac{e}{\sqrt{2 m_{e}}}\left\|\left|\mathbf{A}_{\mathrm{P}}\right|\right\|_{\infty} \leq \sqrt{\frac{1}{2} \hbar \omega_{c}} .
$$

Then the right-hand side of the bound (6.23) is a strictly monotone increasing function of the expectation $\left(\psi, \mathcal{H}_{0}(\boldsymbol{\phi}) \psi\right)$. Therefore, the same argument yields

$$
\mathcal{E}_{n,-}^{\text {edge }} \geq(n+1 / 2) \hbar \omega_{c}-\frac{\sqrt{2} e}{\sqrt{m_{e}}}\left\|\left|\mathbf{A}_{\mathrm{P}}\right|\right\|_{\infty} \sqrt{(n+1 / 2) \hbar \omega_{c}}-\left\|W^{-}\right\|_{\infty}
$$

for $n=0,1,2, \ldots$. If this right-hand side with the index $n+1$ is strictly larger than the right-hand side of (6.24) with the index $n$, then there exists a spectral gap above the Landau band with the index $n$, i.e., $\mathcal{E}_{n+1,-}^{\text {edge }}>\mathcal{E}_{n,+}^{\text {edge }}$. This gap condition can be written as the desired form (2.36) with $\ell=n+1$. Clearly the condition (2.36) is stronger than the above (6.25) for the vector potential $\mathbf{A}_{\mathbf{P}}$. Therefore we have no need to take into account the condition (6.25).

Next we check that the corrections $\delta \sigma_{s y}(t ; \boldsymbol{\phi}, \eta, T)$ in (2.27) and (2.28) to the dominant parts of the linear response coefficients are negligibly small for the slow switching process. Because of the excitation energy gap above the ground state, one can easily prove the bound,

$$
\left|\delta \sigma_{s y}(t ; \phi, \eta, T)\right| \leq \frac{e^{2}}{h} \nu\left[\left(\mathcal{C}_{1}+\mathcal{C}_{2} \omega_{c} T\right) e^{-\eta T}+\left(\mathcal{C}_{3}+\mathcal{C}_{4} \eta / \omega_{c}\right) \eta / \omega_{c}\right],
$$

by using the expressions (4.14)-(4.19). Here all the positive constants $\mathcal{C}_{j}, j=1,2,3,4$, are independent of the system sizes $L_{x}, L_{y}$ and of the number $N$ of the electrons for a fixed filling factor $\nu$ of the electrons.

In the rest of this section, we derive two bounds, (2.37) and (2.38), in Theorem 2.3. To begin with, we rewrite the Hall conductance $\sigma_{x y}(\boldsymbol{\phi})$ of (3.37) as

$$
\sigma_{x y}(\phi)=-\frac{i \hbar e^{2}}{L_{x} L_{y}} \sum_{m, n: \mathcal{E}_{m} \leq \mathcal{E}_{\mathrm{F}}<\mathcal{E}_{n}}\left[\frac{\left\langle\psi_{m}, v_{x}^{(0)} \psi_{n}\right\rangle\left\langle\psi_{n}, v_{y}^{(0)} \psi_{m}\right\rangle}{\left(\mathcal{E}_{m}-\mathcal{E}_{n}\right)^{2}}-(x \leftrightarrow y)\right]
$$

in terms of the eigenvector $\psi_{n}$ of the single electron Hamiltonian $\mathcal{H}(\phi)$ of (6.19) with the energy eigenvalue $\mathcal{E}_{n}$. Here $\mathcal{E}_{\mathrm{F}}$ is the Fermi energy, and

$$
\mathbf{v}^{(0)}=\frac{1}{m_{e}}[\mathbf{p}+e \mathbf{A}(\mathbf{r})] .
$$

We have dropped $\phi$ in $\mathbf{v}^{(0)}$ by relying on the orthogonality between $\psi_{m}$ and $\psi_{n}$ with $\mathcal{E}_{m} \neq \mathcal{E}_{n}$. Further we introduce some operators as follows:

$$
Q\left(\mathcal{E} \leq \mathcal{E}_{\mathrm{F}} ; \boldsymbol{\phi}\right)=\frac{1}{2 \pi i} \int_{\gamma} R(z ; \boldsymbol{\phi}) d z \quad \text { with the resolvent } \quad R(z ; \boldsymbol{\phi})=\frac{1}{z-\mathcal{H}(\boldsymbol{\phi})}
$$

and

$$
Q_{i}\left(\mathcal{E} \leq \mathcal{E}_{\mathrm{F}} ; \boldsymbol{\phi}\right)=\frac{1}{2 \pi i} \int_{\gamma} R(z ; \phi) v_{i}^{(0)} R(z ; \phi) d z
$$


where we have chosen the closed path $\gamma$ so that the operator $Q\left(\mathcal{E} \leq \mathcal{E}_{\mathrm{F}} ; \boldsymbol{\phi}\right)$ is the projection onto the subspace spanned by all the levels below the Fermi energy $\mathcal{E}_{\mathrm{F}}$. Here the path $\gamma$ is taken to be independent of the gauge parameters $\phi$ by the gap condition (2.17). Note that

$$
\begin{aligned}
& \operatorname{Tr}\left[Q\left(\mathcal{E} \leq \mathcal{E}_{\mathrm{F}} ; \boldsymbol{\phi}\right) Q_{x}\left(\mathcal{E} \leq \mathcal{E}_{\mathrm{F}} ; \boldsymbol{\phi}\right) Q_{y}\left(\mathcal{E} \leq \mathcal{E}_{\mathrm{F}} ; \boldsymbol{\phi}\right)\right] \\
= & \sum_{n: \mathcal{E}_{n} \leq \mathcal{E}_{\mathrm{F}}}\left\langle\psi_{n}, Q_{x}\left(\mathcal{E} \leq \mathcal{E}_{\mathrm{F}} ; \boldsymbol{\phi}\right) Q_{y}\left(\mathcal{E} \leq \mathcal{E}_{\mathrm{F}} ; \boldsymbol{\phi}\right) \psi_{n}\right\rangle \\
= & \sum_{n: \mathcal{E}_{n} \leq \mathcal{E}_{\mathrm{F}}}\left\langle\psi_{n}, \frac{1}{2 \pi i} \int_{\gamma} \frac{1}{z_{1}-\mathcal{E}_{n}} v_{x}^{(0)} \frac{1}{z_{1}-\mathcal{H}(\boldsymbol{\phi})} d z_{1} \frac{1}{2 \pi i} \int_{\gamma} \frac{1}{z_{2}-\mathcal{H}(\boldsymbol{\phi})} v_{y}^{(0)} \frac{1}{z_{2}-\mathcal{E}_{n}} d z_{2} \psi_{n}\right\rangle \\
= & \sum_{n: \mathcal{E}_{n} \leq \mathcal{E}_{\mathrm{F}}} \sum_{m}\left\langle\psi_{n}, v_{x}^{(0)} \psi_{m}\right\rangle\left\langle\psi_{m}, v_{y}^{(0)} \psi_{n}\right\rangle\left(\frac{1}{2 \pi i} \int_{\gamma} \frac{1}{z-\mathcal{E}_{n}} \frac{1}{z-\mathcal{E}_{m}} d z\right)^{2} \\
= & \sum_{n: \mathcal{E}_{n} \leq \mathcal{E}_{\mathrm{F}}} \sum_{m: \mathcal{E}_{m}>\mathcal{E}_{\mathrm{F}}}\left\langle\psi_{n}, v_{x}^{(0)} \psi_{m}\right\rangle\left\langle\psi_{m}, v_{y}^{(0)} \psi_{n}\right\rangle \frac{1}{\left(\mathcal{E}_{n}-\mathcal{E}_{m}\right)^{2}} .
\end{aligned}
$$

Combining this with the expression (6.28), the Hall conductance $\sigma_{x y}(\phi)$ can be rewritten as

$$
\sigma_{x y}(\boldsymbol{\phi})=-\frac{i \hbar e^{2}}{L_{x} L_{y}} \operatorname{Tr}\left[Q\left(\mathcal{E} \leq \mathcal{E}_{\mathrm{F}} ; \boldsymbol{\phi}\right)\left\{Q_{x}\left(\mathcal{E} \leq \mathcal{E}_{\mathrm{F}} ; \phi\right) Q_{y}\left(\mathcal{E} \leq \mathcal{E}_{\mathrm{F}} ; \boldsymbol{\phi}\right)-(x \leftrightarrow y)\right\}\right] .
$$

Theorem 6.1 The following inequality is valid:

$$
\left|\sigma_{x y}(\phi+\delta \phi)-\sigma_{x y}(\phi)\right| \leq \mathcal{C} \max _{i=x, y}\left|\delta \phi_{i}\right|
$$

where $\mathcal{C}$ is a positive constant which is independent of the system sizes $L_{x}, L_{y}$ and of the number $N$ of the electrons.

This Theorem follows from the next Proposition 6.2 with the expression $(6.33)$ of $\sigma_{x y}(\phi)$.

Proposition 6.2 The following bound is valid:

$$
\begin{aligned}
& \mid \operatorname{Tr}\left[Q\left(\mathcal{E} \leq \mathcal{E}_{\mathrm{F}} ; \boldsymbol{\phi}+\delta \boldsymbol{\phi}\right) Q_{i}\left(\mathcal{E} \leq \mathcal{E}_{\mathrm{F}} ; \boldsymbol{\phi}+\delta \boldsymbol{\phi}\right) Q_{j}\left(\mathcal{E} \leq \mathcal{E}_{\mathrm{F}} ; \boldsymbol{\phi}+\delta \boldsymbol{\phi}\right)\right] \\
& -\operatorname{Tr}\left[Q\left(\mathcal{E} \leq \mathcal{E}_{\mathrm{F}} ; \boldsymbol{\phi}\right) Q_{i}\left(\mathcal{E} \leq \mathcal{E}_{\mathrm{F}} ; \boldsymbol{\phi}\right) Q_{j}\left(\mathcal{E} \leq \mathcal{E}_{\mathrm{F}} ; \boldsymbol{\phi}\right)\right]\left|\leq \mathcal{C} N \max _{\ell=x, y}\right| \delta \phi_{\ell} \mid
\end{aligned}
$$

for $i, j=x, y$. Here $\mathcal{C}$ is a positive constant which is independent of the system sizes $L_{x}, L_{y}$ and of the number $N$ of the electrons.

The proof is given in Appendix B.

Fix $\phi_{0} \in\left[0, \Delta \phi_{x}\right] \times\left[0, \Delta \phi_{y}\right]$. Then we have

$$
\sigma_{x y}\left(\boldsymbol{\phi}_{0}\right)-\overline{\sigma_{x y}(\boldsymbol{\phi})}=\frac{1}{\Delta \phi_{x} \Delta \phi_{y}} \int_{0}^{\Delta \phi_{x}} d \phi_{x} \int_{0}^{\Delta \phi_{y}} d \phi_{y}\left[\sigma_{x y}\left(\phi_{0}\right)-\sigma_{x y}(\boldsymbol{\phi})\right] .
$$

Using Theorem 6.1, the difference can be evaluated as

$$
\left|\sigma_{x y}\left(\phi_{0}\right)-\overline{\sigma_{x y}(\phi)}\right| \leq \mathcal{C} \max \left\{L_{x}^{-1}, L_{y}^{-1}\right\} .
$$


As shown in Section 5, the averaged Hall conductance is quantized as $\overline{\sigma_{x y}(\phi)}=-e^{2} p / h$ because the present ground state is non-degenerate, i.e., $q=1$. Hence we obtain

$$
\left|\sigma_{x y}\left(\phi_{0}\right)+\frac{e^{2}}{h} p\right| \leq \mathcal{C} \max \left\{L_{x}^{-1}, L_{y}^{-1}\right\}
$$

with an integer $p$.

In order to determine the integer $p$, consider the Hamiltonian

$$
\mathcal{H}(\boldsymbol{\phi}, \boldsymbol{\lambda})=\frac{1}{2 m_{e}}\left[\mathbf{p}+e \mathbf{A}_{0}(\mathbf{r})+e \lambda_{A} \mathbf{A}_{\mathrm{P}}(\mathbf{r})+\boldsymbol{\phi}\right]^{2}+\lambda_{W} W(\mathbf{r})
$$

with the parameters $\boldsymbol{\lambda}=\left(\lambda_{A}, \lambda_{W}\right) \in[0,1]^{2}$. This Hamiltonian $\mathcal{H}(\boldsymbol{\phi}, \boldsymbol{\lambda})$ continuously connects $\mathcal{H}(\phi)$ with $\mathcal{H}_{0}(\phi)$ of $(6.1)$ by varying $\boldsymbol{\lambda}$ from $(1,1)$ to $(0,0)$ continuously. Then, in the same way as in the proof of Proposition 6.2, one can prove that the averaged Hall conductance $\overline{\sigma_{x y}(\phi)}$ for the Hamiltonian $\mathcal{H}(\boldsymbol{\phi}, \boldsymbol{\lambda})$ is a continuous function of the parameters $\boldsymbol{\lambda}$. As shown in Section $6.1, \overline{\sigma_{x y}(\phi)}=-e^{2} \ell / h$ for $\lambda=(0,0)$. Therefore the integer $p$ must be equal to the filling factor $\ell$ of the Landau levels. Consequently we obtain the bound $(2.37)$.

Next consider the acceleration coefficients $\gamma_{s y}(\phi)$. In terms of the projection operator $Q\left(\mathcal{E} \leq \mathcal{E}_{F} ; \phi\right)$, these can be expressed as

$$
\begin{aligned}
\gamma_{s y}(\boldsymbol{\phi}) & =\frac{e^{2}}{L_{x} L_{y}} \frac{\partial}{\partial \phi_{y}} \operatorname{Tr} v_{s}^{(0)}(\boldsymbol{\phi}) Q\left(\mathcal{E} \leq \mathcal{E}_{F} ; \boldsymbol{\phi}\right) \\
& =\frac{e^{2}}{L_{x} L_{y}}\left[\operatorname{Tr} \frac{1}{m_{e}} \delta_{s, y} Q\left(\mathcal{E} \leq \mathcal{E}_{F} ; \boldsymbol{\phi}\right)+\operatorname{Tr} v_{s}^{(0)}(\boldsymbol{\phi}) Q_{y}\left(\mathcal{E} \leq \mathcal{E}_{F} ; \boldsymbol{\phi}\right)\right] \\
& =\frac{e^{2}}{L_{x} L_{y}}\left[\frac{N}{m_{e}} \delta_{s, y}+\operatorname{Tr} v_{s}^{(0)}(\boldsymbol{\phi}) Q_{y}\left(\mathcal{E} \leq \mathcal{E}_{F} ; \boldsymbol{\phi}\right)\right] .
\end{aligned}
$$

Theorem 6.3 The following inequality is valid:

$$
\left|\gamma_{s y}(\phi+\delta \phi)-\gamma_{s y}(\phi)\right| \leq \mathcal{C} \max _{i=x, y}\left|\delta \phi_{i}\right|,
$$

where the positive constant $\mathcal{C}$ is independent of the time $t$, the number $N$ of the electrons, and of the system sizes $L_{x}, L_{y}$.

Owing to the expression (6.40), this theorem follows from the proposition:

Proposition 6.4 The following inequality is valid:

$$
\left|\operatorname{Tr} v_{s}^{(0)}(\boldsymbol{\phi}+\delta \boldsymbol{\phi}) Q_{y}\left(\mathcal{E} \leq \mathcal{E}_{F} ; \boldsymbol{\phi}+\delta \boldsymbol{\phi}\right)-\operatorname{Tr} v_{s}^{(0)}(\boldsymbol{\phi}) Q_{y}\left(\mathcal{E} \leq \mathcal{E}_{F} ; \boldsymbol{\phi}\right)\right| \leq \mathcal{C} N \max _{i=x, y}\left|\delta \phi_{i}\right|,
$$

where the positive constant $\mathcal{C}$ is independent of the time $t$, the number $N$ of the electrons, and of the system sizes $L_{x}, L_{y}$.

The proof is given in Appendix $\mathrm{C}$.

Fix $\phi_{0} \in\left[0, \Delta \phi_{x}\right] \times\left[0, \Delta \phi_{y}\right]$. Then

$$
\gamma_{s y}\left(\phi_{0}\right)-\overline{\gamma_{s y}(\boldsymbol{\phi})}=\frac{1}{\Delta \phi_{x} \Delta \phi_{y}} \int_{0}^{\Delta \phi_{x}} d \phi_{x} \int_{0}^{\Delta \phi_{y}} d \phi_{y}\left[\gamma_{s y}\left(\phi_{0}\right)-\gamma_{s y}(\boldsymbol{\phi})\right] \text {. }
$$

Using Theorem 6.3 and the fact $\overline{\gamma_{s y}(\phi)}=0$ which was shown in Section 5, we have the bound (2.38). 


\section{The interacting case}

In this section we study the interacting case in detail. In Sec. 5, we obtained that the averaged Hall conductance is quantized as

$$
\overline{\sigma_{x y}(\phi)}=-\frac{e^{2}}{h} \frac{p}{q}
$$

with integers $p, q$. Here the integer $q$ is the dimension of the sector of the ground state. Since the Hall conductance $\sigma_{x y}(\phi)$ is a continuous function of the gauge parameters $\phi$, we can find special gauge parameters $\phi_{0} \in \mathcal{T}_{\mathrm{g}}$ satisfying

$$
\sigma_{x y}\left(\phi_{0}\right)=-\frac{e^{2}}{h} \frac{p}{q},
$$

by using the mean value theorem about integration. Namely the Hall conductance is exactly quantized for the special value $\phi=\phi_{0}$ of the gauge parameters. But we cannot necessarily expect the same exact quantization for general fixed values of the gauge parameters in $\mathcal{T}_{\mathrm{g}}$. Besides, the value $\phi_{0}$ may strongly depend on the potentials, the system sizes $L_{x}, L_{y}$ and on the number $N$ of the electrons although $\phi_{0}$ tends to zero as $L_{x}, L_{y} \rightarrow \infty .{ }^{14}$

As we treated the non-interacting case in the preceding section, we want to resolve the following two issues for the interacting case:

- Estimating the finite size correction for $\sigma_{x y}(\phi)$ for fixed gauge parameters $\phi \neq \phi_{0}$.

- Elucidating the relation between the integer $p$ and the filling factor $\nu$ of the Landau levels.

Unfortunately we cannot estimate the finite size correction because of certain technical difficulty. Therefore we focus on the second issue only in this paper.

\subsection{Boundedness of the Hall conductance $\sigma_{x y}(\phi)$}

Firstly we prove that the Hall conductance $\sigma_{x y}(\phi)$ is uniformly bounded in the number $N$ of the electrons and the system sizes $L_{x}, L_{y}$ for any fixed filling factor $\nu$ under the assumptions below. If the dimension $q$ of the sector of the ground state is also uniformly bounded in $L_{x}, L_{y}, N$ in addition to the boundedness of $\sigma_{x y}(\phi)$, then there exist a sequence $\left\{\left(L_{x}^{(i)}, L_{y}^{(i)}\right)\right\}_{i}$ of the system sizes going to infinity and two integers $p^{(\infty)}, q^{(\infty)}$ such that

$$
\sigma_{x y}^{(\infty)}:=\lim _{L_{x}^{(i)}, L_{y}^{(i)} \rightarrow \infty} \sigma_{x y}\left(\phi_{0}\right)=-\frac{e^{2}}{h} \frac{p^{(\infty)}}{q^{(\infty)}} .
$$

Namely the quantization of the Hall conductance occurs in the infinite volume limit although the number $p^{(\infty)} / q^{(\infty)}$ may be equal to an integer. Unfortunately we cannot determine the explicit values of the integers $p^{(\infty)}$ and $q^{(\infty)}$ for the infinite volume ground state for a given filling factor $\nu$.

In order to prove the boundedness of the Hall conductance $\sigma_{x y}(\boldsymbol{\phi})$, we need some technical assumptions:

\footnotetext{
${ }^{14}$ The space $\mathcal{T}_{\mathrm{g}}$ itself contracts into the single point $(0,0)$ in the infinite volume limit from the definition (2.5) of the gauge torus $\mathcal{T}_{\mathrm{g}}$.
} 
Assumption 7.1 The the electrostatic potential $W$ and the electron-electron interaction $W^{(2)}$ of the present model satisfy higher differentiability as $W \in C^{2}\left(\mathbf{R}^{2}\right)$ and $W^{(2)} \in$ $C^{1}\left(\mathbf{R}^{2}\right)$. The norms,

$$
\left\|\frac{\partial^{2} W}{\partial x^{2}}\right\|_{\infty} \text { and }\left\|\frac{\partial^{2} W}{\partial y^{2}}\right\|_{\infty},
$$

are bounded uniformly in the sizes $L_{x}, L_{y}$ of the system.

Theorem 7.2 Suppose $\mathbf{A}_{\mathrm{P}}=0$, and require Assumption 7.1 in addition to the assumptions (including Assumption 2.1) in Sec. 2. Then the the Hall conductance $\sigma_{x y}(\phi)$ of (3.37) is uniformly bounded in the number $N$ of the electrons and in the system sizes $L_{x}, L_{y}$ for any fixed filling factor $\nu$.

The proof is given in Appendix D. In the same way, we can prove the boundedness of the acceleration coefficients $\gamma_{s y}(\boldsymbol{\phi})$ of (3.38) and (3.43), and can get the bound,

$$
\left|\delta \sigma_{s y}(t ; \phi, \eta, T)\right| \leq \frac{e^{2}}{h} \nu\left[\left(\mathcal{C}_{1}+\mathcal{C}_{2} \omega_{c} T\right) e^{-\eta T}+\left(\mathcal{C}_{3}+\mathcal{C}_{4} \eta / \omega_{c}\right) \eta / \omega_{c}\right]
$$

for the corrections $\delta \sigma_{s y}(t ; \boldsymbol{\phi}, \eta, T)$ to the dominant parts of the linear response coefficients (2.27) and (2.28) under the same assumptions. Here all the positive constants $\mathcal{C}_{j}, j=$ $1,2,3,4$, are independent of the system sizes $L_{x}, L_{y}$ and of the number $N$ of the electrons for a fixed filling factor $\nu$ of the electrons.

Next consider the case with $\mathbf{A}_{\mathrm{P}} \neq 0$. We write the $z$ component of the magnetic field for the vector potential $\mathbf{A}_{\mathrm{P}}$ as

$$
B_{\mathrm{P}, z}=\frac{\partial A_{\mathrm{P}, y}}{\partial x}-\frac{\partial A_{\mathrm{P}, x}}{\partial y}
$$

Assumption 7.3 The magnetic field $B_{\mathrm{P}, z}$ for the vector potential $\mathbf{A}_{\mathrm{P}}$ satisfies $B_{\mathrm{P}, z} \in$ $C^{2}\left(\mathbf{R}^{2}\right)$, and the norms,

$$
\left\|B_{\mathrm{P}, z}\right\|_{\infty},\left\|\frac{\partial B_{\mathrm{P}, z}}{\partial x}\right\|_{\infty} \quad \text { and } \quad\left\|\frac{\partial B_{\mathrm{P}, z}}{\partial y}\right\|_{\infty},
$$

are bounded uniformly in the sizes $L_{x}, L_{y}$ of the system.

Assumption 7.4 The electron-electron interaction $W^{(2)}$ is non-negative (repulsive) and satisfies the decay condition,

$$
W^{(2)}(x, y) \leq W_{0}^{(2)}\left\{1+\left[\operatorname{dist}(x, y) / r_{0}\right]^{2}\right\}^{-\gamma / 2} \quad \text { with the constants } W_{0}^{(2)}>0, \gamma>2, r_{0}>0
$$

where the distance is given by

$$
\operatorname{dist}(x, y):=\sqrt{\min _{m \in \mathbf{Z}}\left|x-m L_{x}\right|^{2}+\min _{n \in \mathbf{Z}}\left|y-n L_{y}\right|^{2}} .
$$

For the case with $\mathbf{A}_{\mathrm{P}} \neq 0$, we have the following theorem: 
Theorem 7.5 Require Assumptions 7.1, 7.3 and 7.4 in addition to the assumptions (including Assumption 2.1) in Sec. 2. Then the Hall conductance $\sigma_{x y}(\phi)$ of (3.37) is uniformly bounded in the number $N$ of the electrons and in the system sizes $L_{x}, L_{y}$ for any fixed filling factor $\nu$ of the electrons.

The proof is given in Appendix D. Under the same assumptions, we can also prove the boundedness of the acceleration coefficients $\gamma_{s y}(\phi)$, and can get the same bound for $\delta \sigma_{s y}(t, \boldsymbol{\phi} ; \eta, T)$ as $(7.5)$ with different constants $\mathcal{C}_{j}$.

\subsection{Fractional quantization of the Hall conductance $\sigma_{x y}(\phi)$}

The rational number $p / q$ in the right-hand side of (7.1) or (7.2) may be equal to an integer, i.e., the number $p$ may equal a multiple of $q$. But, from the result (4.2), we can expect that the Hall conductance exhibits purely fractional quantization as

$$
\sigma_{x y}\left(\phi_{0}\right)=-\frac{e^{2}}{h} \nu
$$

for a fractional filling factor $\nu=p / q \notin \mathbf{N}$ when a spectral gap exists above the sector of the ground state with weak disorder. Next we shall show that this expectation holds under certain assumptions.

When the system is translationally invariant in one direction [27], we can obtain the desired result as follows:

Theorem 7.6 In addition to the assumptions (including Assumption 2.1) in Sec. 2, assume that the electrostatic potential $W$ is a function of the single variable $x$ only or $y$ only as $W(x)$ or $W(y)$, and assume $\mathbf{A}_{\mathrm{P}}=0, W \in C^{1}(\mathbf{R})$ and $W^{(2)} \in C^{1}\left(\mathbf{R}^{2}\right)$. Then there exist gauge parameters $\phi_{0} \in \mathcal{T}_{\mathrm{g}}$ such that

$$
\sigma_{x y}\left(\phi_{0}\right)=-\frac{e^{2}}{h} \frac{p}{q}
$$

for the fractional filling factor $\nu=p / q$ of the Landau levels.

The proof is given in Appendix E.

In order to proceed to more generic situations, we write

$$
D^{(m, n)}:=\frac{\partial^{m+n}}{\partial x^{m} \partial y^{n}}
$$

for non-negative integers $m, n$.

Assumption 7.7 The electrostatic potential $W$ and the electron-electron interaction $W^{(2)}$ satisfy higher differentiability as $W \in C^{3}\left(\mathbf{R}^{2}\right)$ and $W^{(2)} \in C^{1}\left(\mathbf{R}^{2}\right)$. 
Theorem 7.8 Suppose $\mathbf{A}_{\mathrm{P}}=0$, i.e., $B_{\mathrm{P}, z}=0$, and require Assumption 7.7 in addition to the assumptions (including Assumption 2.1) in Sec. 2. Then the Hall conductance $\overline{\sigma_{x y}(\boldsymbol{\phi})}$ averaged over the gauge parameters $\phi$ satisfies the bound,

$$
-\frac{e^{2}}{h} \nu(1+\delta) \leq \overline{\sigma_{x y}(\phi)} \leq-\frac{e^{2}}{h} \nu(1-\delta)
$$

with

$$
\delta=2 \ell_{B}^{4} \frac{\hbar \omega_{c}}{(\Delta E)^{3}} \max _{m+n=2}\left\|D^{(m, n)} W\right\|_{\infty}^{2}
$$

Here $\nu=N / M$ is the fixed filling factor of the electrons, and the definition of the energy gap $\Delta E$ is given in Assumption 2.1.

The proof is given in Appendix E. We can obtain a similar bound for the non-averaged Hall conductance $\sigma_{x y}(\phi)$ to (7.13). See Appendix E.

Corollary 7.9 Under the same assumptions as in the above theorem, there exist gauge parameters $\phi_{0} \in \mathcal{T}_{\mathrm{g}}$ such that

$$
\sigma_{x y}\left(\phi_{0}\right)=-\frac{e^{2}}{h} \frac{p}{q}
$$

with the integers $p, q$ satisfying

$$
\nu(1-\delta) \leq \frac{p}{q} \leq \nu(1+\delta)
$$

with the same $\delta$ as in the theorem.

Consider again the situation that the interval $[\nu(1-\delta), \nu(1+\delta)]$ does not include any integer. Then the number $p / q$ must be equal to purely a fractional number, i.e., a noninteger. In addition to this condition, if the number $\delta$ and the dimension $q$ of the sector of the ground state are uniformly bounded in the sizes $L_{x}, L_{y}$ of the system, then there exist a sequence $\left\{\left(L_{x}^{(i)}, L_{y}^{(i)}\right)\right\}_{i}$ of the system sizes going to infinity and two integers $p^{(\infty)}, q^{(\infty)}$ such that

$$
\sigma_{x y}^{(\infty)}:=\lim _{L_{x}^{(i)}, L_{y}^{(i)} \rightarrow \infty} \sigma_{x y}\left(\phi_{0}\right)=-\frac{e^{2}}{h} \frac{p^{(\infty)}}{q^{(\infty)}},
$$

and that $\sigma_{x y}^{(\infty)}$ satisfies the bound derived from (7.16). Therefore the number $p^{(\infty)} / q^{(\infty)}$ equals a purely fractional number also in the infinite volume limit.

In order to get a similar bound for the Hall conductance in the case with the vector potential $\mathbf{A}_{\mathrm{P}} \neq 0$, we need stronger assumptions as follows:

Assumption 7.10 The magnetic field $B_{\mathrm{P}, z}$ for the vector potential $\mathbf{A}_{\mathrm{P}}$ and the electrostatic potential $W$ of the present model satisfy higher differentiability as $B_{\mathrm{P}, z} \in C^{4}\left(\mathbf{R}^{2}\right)$ and $W \in C^{4}\left(\mathbf{R}^{2}\right)$. 
Assumption 7.11 The electron-electron interaction $W^{(2)}$ of the present model satisfies $W^{(2)} \in C^{2}\left(\mathbf{R}^{2}\right)$, and the following two conditions:

$$
\ell_{B}\left[\left|\left(\frac{\partial W^{(2)}}{\partial x}\right)(\mathbf{r})\right|+\left|\left(\frac{\partial W^{(2)}}{\partial y}\right)(\mathbf{r})\right|\right] \leq \alpha_{\mathrm{int}} W^{(2)}(\mathbf{r})
$$

and

$$
\ell_{B}^{2}\left[\left|\left(\frac{\partial^{2} W^{(2)}}{\partial x^{2}}\right)(\mathbf{r})\right|+\left|\left(\frac{\partial^{2} W^{(2)}}{\partial y^{2}}\right)(\mathbf{r})\right|\right] \leq \alpha_{\text {int }} W^{(2)}(\mathbf{r})
$$

for any $\mathbf{r}$, with a positive constant $\alpha_{\text {int }}$ which is independent of the sizes $L_{x}, L_{y}$ of the system.

Theorem 7.12 In addition to the assumptions (including Assumption 2.1) in Sec. 2, we require Assumptions 7.4, 7.10 and 7.11. Then there exists a positive number $\delta$ such that $\delta$ is a continuous function of the norms, $\left\|D^{(k, \ell)} B_{\mathrm{P}, z}\right\|_{\infty}$ for $k+\ell \leq 4$ and $\left\|D^{(m, n)} W\right\|_{\infty}$ for $m+n \leq 3$, and satisfies $\delta=0$ for the special point with $\mathbf{A}_{\mathrm{P}}=0$ and $W=0$, and that the Hall conductance $\overline{\sigma_{x y}(\phi)}$ satisfies the bound,

$$
-\frac{e^{2}}{h} \nu(1+\delta) \leq \overline{\sigma_{x y}(\phi)} \leq \frac{e^{2}}{h} \nu(1-\delta),
$$

where $\nu=N / M$ is the fixed filling factor of the electrons.

The proof is given in Appendix E. Clearly we get the following corollary similar to Corollary 7.9:

Corollary 7.13 Under the same assumptions as in the above theorem, there exists gauge parameters $\phi_{0} \in \mathcal{T}_{\mathrm{g}}$ such that

$$
\sigma_{x y}\left(\phi_{0}\right)=-\frac{e^{2}}{h} \frac{p}{q}
$$

with integers $p, q$ satisfying

$$
\nu(1-\delta) \leq \frac{p}{q} \leq \nu(1+\delta)
$$

with the same $\delta$ as in the above theorem.

For this case, we can also make the same remarks as those after Corollary 7.9. But we omit to make those remarks again.

\section{A Differentiability of the ground-state wavefunctions}

Following Kato, ${ }^{15}$ we give the proof of Proposition 5.1 in this appendix. For this purpose, it is enough to construct an operator-valued function $U_{\mathrm{g}}(\phi)$ of the gauge parameters $\phi \in \mathcal{T}_{\mathrm{g}}$ with the following two conditions:

\footnotetext{
${ }^{15}$ See Sec. 4.2 of Chap. II of his book [47].
} 
- The inverse $U_{\mathrm{g}}^{-1}(\boldsymbol{\phi})$ exists and both of $U_{\mathrm{g}}(\boldsymbol{\phi})$ and $U_{\mathrm{g}}^{-1}(\boldsymbol{\phi})$ are infinitely differentiable with respect to $\phi$;

- $U_{\mathrm{g}}(\boldsymbol{\phi}) Q\left(E_{0}^{(N)}(0)\right) U_{\mathrm{g}}^{-1}(\boldsymbol{\phi})=Q\left(E_{0}^{(N)}(\boldsymbol{\phi})\right)$.

From this second property, one obtain that, if the vectors $\hat{\Phi}_{0, \mu}^{(N)}(0), \mu=1,2, \ldots, q$, span the sector of the degenerate ground state for $\phi=0$, then the vectors $\hat{\Phi}_{0, \mu}^{(N)}(\phi)=U_{\mathrm{g}}(\phi) \hat{\Phi}_{0, \mu}^{(N)}(0)$, $\mu=1,2, \ldots, q$, also span the sector of the degenerate ground state for any given $\phi \in \mathcal{T}_{\mathrm{g}}$.

We begin with the following lemma:

Lemma A.1 Let $z \notin \sigma\left(H_{0}^{(N)}(\phi)\right)$, i.e., the complex number $z$ is not in the spectrum $\sigma\left(H_{0}^{(N)}(\boldsymbol{\phi})\right)$ of the Hamiltonian $H_{0}^{(N)}(\boldsymbol{\phi})$. Then

$$
\begin{aligned}
\leq & \left\|\frac{1}{m_{e}}\left[p_{s, j}+e A_{s}\left(\mathbf{r}_{j}\right)+\phi_{s}\right] \frac{1}{z-H_{0}^{(N)}(\boldsymbol{\phi})}\right\| \\
\leq & \sqrt{\frac{2}{m_{e} \operatorname{dist}\left(z, \sigma\left(H_{0}^{(N)}(\boldsymbol{\phi})\right)\right)}\left[1+\frac{|z|+N\|W\|_{\infty}+N(N-1)\left\|W^{(2)}\right\|_{\infty} / 2}{\operatorname{dist}\left(z, \sigma\left(H_{0}^{(N)}(\boldsymbol{\phi})\right)\right)}\right]}
\end{aligned}
$$

for $s=x, y$ and $j=1,2, \ldots, N$.

Proof: Let $\Phi$ be an $N$ electrons vector with norm one. Then one has

$$
\begin{aligned}
& \left\langle\Phi, \frac{1}{z^{*}-H_{0}^{(N)}(\boldsymbol{\phi})}\left\{\frac{1}{m_{e}}\left[p_{s, j}+e A_{s}\left(\mathbf{r}_{j}\right)+\phi_{s}\right]\right\}^{2} \frac{1}{z-H_{0}^{(N)}(\boldsymbol{\phi})} \Phi\right\rangle \\
\leq & \left\langle\Phi, \frac{1}{z^{*}-H_{0}^{(N)}(\boldsymbol{\phi})} \frac{2}{m_{e}}\left[H_{0}^{(N)}(\boldsymbol{\phi})+N\|W\|_{\infty}+\frac{N(N-1)}{2}\left\|W^{(2)}\right\|_{\infty}\right] \frac{1}{z-H_{0}^{(N)}(\boldsymbol{\phi})} \Phi\right\rangle \\
= & \frac{2}{m_{e}} \sum_{n}\left|\left\langle\Phi, \Phi_{n}^{(N)}(\boldsymbol{\phi})\right\rangle\right|^{2} \frac{1}{\left|z-E_{n}^{(N)}(\boldsymbol{\phi})\right|^{2}}\left[E_{n}^{(N)}(\boldsymbol{\phi})+N\|W\|_{\infty}+\frac{N(N-1)}{2}\left\|W^{(2)}\right\|_{\infty}\right],
\end{aligned}
$$

where $\Phi_{n}^{(N)}(\phi)$ are the eigenvectors of $H_{0}^{(N)}(\phi)$ with the eigenvalue $E_{n}^{(N)}(\phi)$ counting degenerate eigenvalues a number of times equal to their multiplicity. Note that

$$
\begin{aligned}
& \frac{1}{\left|z-E_{n}^{(N)}(\phi)\right|^{2}}\left[E_{n}^{(N)}(\phi)+N\|W\|_{\infty}+\frac{N(N-1)}{2}\left\|W^{(2)}\right\|_{\infty}\right] \\
= & \frac{1}{\left|z-E_{n}^{(N)}(\phi)\right|^{2}}\left[E_{n}^{(N)}(\phi)-z+z+N\|W\|_{\infty}+\frac{N(N-1)}{2}\left\|W^{(2)}\right\|_{\infty}\right] \\
= & -\frac{1}{z^{*}-E_{n}^{(N)}(\phi)}+\frac{1}{\left|z-E_{n}^{(N)}(\phi)\right|^{2}}\left[z+N\|W\|_{\infty}+\frac{N(N-1)}{2}\left\|W^{(2)}\right\|_{\infty}\right] \\
\leq & \frac{1}{\operatorname{dist}\left(z, \sigma\left(H_{0}^{(N)}(\phi)\right)\right)}+\frac{|z|+N\|W\|_{\infty}+N(N-1)\left\|W^{(2)}\right\|_{\infty} / 2}{\operatorname{dist}\left(z, \sigma\left(H_{0}^{(N)}(\phi)\right)\right)^{2}} .
\end{aligned}
$$

Substituting this into (A.2), the desired bound (A.1) is obtained. 
For any $z \notin \sigma\left(H_{0}^{(N)}(\phi)\right)$, one has

$$
\frac{\partial}{\partial \phi_{s}} R^{(N)}(z ; \boldsymbol{\phi})=\sum_{j=1}^{N} R^{(N)}(z ; \boldsymbol{\phi}) \frac{1}{m_{e}}\left[p_{s, j}+e A_{s}\left(\mathbf{r}_{j}\right)+\phi_{s}\right] R^{(N)}(z ; \boldsymbol{\phi}),
$$

where we have written

$$
R^{(N)}(z ; \phi)=\frac{1}{z-H_{0}^{(N)}(\phi)} .
$$

Here, since the product $\left[p_{s, j}+e A_{s}\left(\mathbf{r}_{j}\right)+\phi_{s}\right] R^{(N)}(z ; \boldsymbol{\phi})$ of the two operators is bounded owing to the above Lemma A.1, the resolvent $R^{(N)}(z ; \phi)$ is infinitely differentiable with respect to $\phi$. Therefore, by the integral representation $(5.4)$, the projection $Q\left(E_{0}^{(N)}(\phi)\right)$ is also infinitely differentiable with respect to $\phi$.

We introduce abbreviations as

$$
P(\phi)=Q\left(E_{0}^{(N)}(\phi)\right)
$$

and

$$
P_{s}(\phi)=\frac{\partial}{\partial \phi_{s}} Q\left(E_{0}^{(N)}(\phi)\right) \quad \text { for } s=x, y,
$$

and write the commutators for them as

$$
F^{(s)}(\phi)=\left[P_{s}(\phi), P(\phi)\right]
$$

Note that, for $s=x, y$,

$$
P_{s}(\phi) P(\phi)+P(\phi) P_{s}(\phi)=P_{s}(\phi)
$$

and

$$
P(\phi) P_{s}(\phi) P(\phi)=0 .
$$

These are equivalent to the equations (5.9) and (5.10), respectively. Combining the latter with (A.8), one has

$$
P(\phi) F^{(s)}(\phi)=-P(\phi) P_{s}(\phi), \quad F^{(s)}(\phi) P(\phi)=P_{s}(\phi) P(\phi) .
$$

Further, from these and (A.9), one obtains

$$
P_{s}(\phi)=\left[F^{(s)}(\phi), P(\phi)\right]
$$

Now introduce the ordinary differential equation,

$$
\frac{d}{d \phi_{x}} X_{+}=F^{(x)}\left(\phi_{x}, 0\right) X_{+}
$$

for the unknown operator-valued function $X_{+}=X_{+}\left(\phi_{x}\right)$ of $\phi_{x}$. Here we have fixed the argument $\phi_{y}$ to zero in $F^{(x)}$. Let $X_{+}=U_{\mathrm{g}}^{(x)}\left(\phi_{x}\right)$ be the unique solution satisfying the initial condition $U_{\mathrm{g}}^{(x)}(0)=1$. Since $F^{(x)}\left(\phi_{x}, 0\right)$ is infinitely differentiable with respect to $\phi_{x}$, the solution $U_{\mathrm{g}}^{(x)}\left(\phi_{x}\right)$ is also infinitely differentiable. The existence and the infinite 
differentiability of the unique solution follow from the standard theory for differential equations. Further introduce the ordinary differential equation,

$$
\frac{d}{d \phi_{x}} X_{-}=-X_{-} F^{(x)}\left(\phi_{x}, 0\right),
$$

for the unknown operator-valued function $X_{-}=X_{-}\left(\phi_{x}\right)$. Let $X_{-}=V_{\mathrm{g}}^{(x)}\left(\phi_{x}\right)$ be the unique solution satisfying the initial condition $V_{\mathrm{g}}^{(x)}(0)=1$.

Note that

$$
\begin{aligned}
& \frac{d}{d \phi_{x}}\left(V_{\mathrm{g}}^{(x)}\left(\phi_{x}\right) U_{\mathrm{g}}^{(x)}\left(\phi_{x}\right)\right) \\
= & \frac{d V_{\mathrm{g}}^{(x)}}{d \phi_{x}}\left(\phi_{x}\right) U_{\mathrm{g}}^{(x)}\left(\phi_{x}\right)+V_{\mathrm{g}}^{(x)}\left(\phi_{x}\right) \frac{d U_{\mathrm{g}}^{(x)}}{d \phi_{x}}\left(\phi_{x}\right) \\
= & -V_{\mathrm{g}}^{(x)}\left(\phi_{x}\right) F^{(x)}\left(\phi_{x}, 0\right) U_{\mathrm{g}}^{(x)}\left(\phi_{x}\right)+V_{\mathrm{g}}^{(x)}\left(\phi_{x}\right) F^{(x)}\left(\phi_{x}, 0\right) U_{\mathrm{g}}^{(x)}\left(\phi_{x}\right)=0 .
\end{aligned}
$$

Hence $V_{\mathrm{g}}^{(x)} U_{\mathrm{g}}^{(x)}$ is a constant and

$$
V_{\mathrm{g}}^{(x)}\left(\phi_{x}\right) U_{\mathrm{g}}^{(x)}\left(\phi_{x}\right)=V_{\mathrm{g}}^{(x)}(0) U_{\mathrm{g}}^{(x)}(0)=1 \quad \text { for all } \phi_{x}
$$

Similarly one has

$$
\begin{aligned}
& \frac{d}{d \phi_{x}}\left(U_{\mathrm{g}}^{(x)}\left(\phi_{x}\right) V_{\mathrm{g}}^{(x)}\left(\phi_{x}\right)\right) \\
= & \frac{d U_{\mathrm{g}}^{(x)}}{d \phi_{x}}\left(\phi_{x}\right) V_{\mathrm{g}}^{(x)}\left(\phi_{x}\right)+U_{\mathrm{g}}^{(x)}\left(\phi_{x}\right) \frac{d V_{\mathrm{g}}^{(x)}}{d \phi_{x}}\left(\phi_{x}\right) \\
= & F^{(x)}\left(\phi_{x}, 0\right) U_{\mathrm{g}}^{(x)}\left(\phi_{x}\right) V_{\mathrm{g}}^{(x)}\left(\phi_{x}\right)+U_{\mathrm{g}}^{(x)}\left(\phi_{x}\right)\left[-V_{\mathrm{g}}^{(x)}\left(\phi_{x}\right) F^{(x)}\left(\phi_{x}, 0\right)\right] \\
= & {\left[F^{(x)}\left(\phi_{x}, 0\right), U_{\mathrm{g}}^{(x)}\left(\phi_{x}\right) V_{\mathrm{g}}^{(x)}\left(\phi_{x}\right)\right] . }
\end{aligned}
$$

This is also an ordinary differential equation for $Z\left(\phi_{x}\right)=U_{\mathrm{g}}^{(x)}\left(\phi_{x}\right) V_{\mathrm{g}}^{(x)}\left(\phi_{x}\right)$ with the initial condition $Z(0)=U_{\mathrm{g}}^{(x)}(0) V_{\mathrm{g}}^{(x)}(0)=1$. Clearly $Z\left(\phi_{x}\right)=1$ satisfies the equation and the initial condition. Combining this with the uniqueness of the solution, the following relation must hold:

$$
U_{\mathrm{g}}^{(x)}\left(\phi_{x}\right) V_{\mathrm{g}}^{(x)}\left(\phi_{x}\right)=1 \quad \text { for all } \phi_{x}
$$

By combining this with (A.16), one gets $V_{\mathrm{g}}^{(x)}=\left(U_{\mathrm{g}}^{(x)}\right)^{-1}$.

Consider the operator-valued function $P\left(\phi_{x}, 0\right) U_{\mathrm{g}}^{(x)}\left(\phi_{x}\right)$, and by differentiation, one has

$$
\begin{aligned}
\frac{d}{d \phi_{x}}\left(P\left(\phi_{x}, 0\right) U_{\mathrm{g}}^{(x)}\left(\phi_{x}\right)\right) & =P_{x}\left(\phi_{x}, 0\right) U_{\mathrm{g}}^{(x)}\left(\phi_{x}\right)+P\left(\phi_{x}, 0\right) \frac{d U_{\mathrm{g}}^{(x)}}{d \phi_{x}}\left(\phi_{x}\right) \\
& =P_{x}\left(\phi_{x}, 0\right) U_{\mathrm{g}}^{(x)}\left(\phi_{x}\right)+P\left(\phi_{x}, 0\right) F^{(x)}\left(\phi_{x}, 0\right) U_{\mathrm{g}}^{(x)}\left(\phi_{x}\right) \\
& =\left[P_{x}\left(\phi_{x}, 0\right)+P\left(\phi_{x}, 0\right) F^{(x)}\left(\phi_{x}, 0\right)\right] U_{\mathrm{g}}^{(x)}\left(\phi_{x}\right) \\
& =F^{(x)}\left(\phi_{x}, 0\right) P\left(\phi_{x}, 0\right) U_{\mathrm{g}}^{(x)}\left(\phi_{x}\right),
\end{aligned}
$$


where we have used the relation (A.12). Thus the function $X_{+}=P\left(\phi_{x}, 0\right) U_{\mathrm{g}}^{(x)}\left(\phi_{x}\right)$ is a solution of the differential equation (A.13) with the initial condition $P(0,0) U_{\mathrm{g}}^{(x)}(0)=P(0,0)$. By the uniqueness of the solution, $P\left(\phi_{x}, 0\right) U_{\mathrm{g}}^{(x)}\left(\phi_{x}\right)$ must coincide with $U_{\mathrm{g}}^{(x)}\left(\phi_{x}\right) P(0,0)$ which has the same initial condition $X_{+}(0)=P(0,0)$. Namely one has

$$
P\left(\phi_{x}, 0\right) U_{\mathrm{g}}^{(x)}\left(\phi_{x}\right)=U_{\mathrm{g}}^{(x)}\left(\phi_{x}\right) P(0,0) .
$$

Next consider the ordinary differential equation,

$$
\frac{d}{d \phi_{y}} Y_{+}=F^{(y)}\left(\phi_{x}, \phi_{y}\right) Y_{+},
$$

for the unknown operator-valued function $Y_{+}=Y_{+}\left(\phi_{y} ; \phi_{x}\right)$ of $\phi_{y}$ and with the parameter $\phi_{x}$. Let $Y_{+}=U_{\mathrm{g}}^{(y)}\left(\phi_{x}, \phi_{y}\right)$ be the unique solution satisfying the initial condition $U_{\mathrm{g}}^{(y)}\left(\phi_{x}, 0\right)=1$. The solution $U_{\mathrm{g}}^{(y)}\left(\phi_{x}, \phi_{y}\right)$ is infinitely differentiable with respect to both $\phi_{x}$ and $\phi_{y}$ because of the infinitely differentiability of $F^{(y)}$. Further introduce the ordinary differential equation,

$$
\frac{d}{d \phi_{y}} Y_{-}=-Y_{-} F^{(y)}\left(\phi_{x}, \phi_{y}\right)
$$

for the unknown operator-valued function $Y_{-}=Y_{-}\left(\phi_{y} ; \phi_{x}\right)$ of $\phi_{y}$ and with the parameter $\phi_{x}$. Let $Y_{-}=V_{\mathrm{g}}^{(y)}\left(\phi_{x}, \phi_{y}\right)$ be the unique solution satisfying the initial condition $V_{\mathrm{g}}^{(y)}\left(\phi_{x}, 0\right)=1$. Then one has $V_{\mathrm{g}}^{(y)}=\left(U_{\mathrm{g}}^{(y)}\right)^{-1}$ in the same way as in the above. Consider the function $P\left(\phi_{x}, \phi_{y}\right) U_{\mathrm{g}}^{(y)}\left(\phi_{x}, \phi_{y}\right)$. By differentiation, one has

$$
\begin{aligned}
\frac{\partial}{\partial \phi_{y}}\left(P\left(\phi_{x}, \phi_{y}\right) U_{\mathrm{g}}^{(y)}\left(\phi_{x}, \phi_{y}\right)\right) & =P_{y}\left(\phi_{x}, \phi_{y}\right) U_{\mathrm{g}}^{(y)}\left(\phi_{x}, \phi_{y}\right)+P\left(\phi_{x}, \phi_{y}\right) \frac{\partial U_{\mathrm{g}}^{(y)}}{\partial \phi_{y}}\left(\phi_{x}, \phi_{y}\right) \\
& =\left[P_{y}\left(\phi_{x}, \phi_{y}\right)+P\left(\phi_{x}, \phi_{y}\right) F^{(y)}\left(\phi_{x}, \phi_{y}\right)\right] U_{\mathrm{g}}^{(y)}\left(\phi_{x}, \phi_{y}\right) \\
& =F^{(y)}\left(\phi_{x}, \phi_{y}\right) P\left(\phi_{x}, \phi_{y}\right) U_{\mathrm{g}}^{(y)}\left(\phi_{x}, \phi_{y}\right),
\end{aligned}
$$

where we have used the relation (A.12). This equation may be treated as the ordinary differential equation (A.21) for the function $Y_{+}\left(\phi_{y} ; \phi_{x}\right)=P\left(\phi_{x}, \phi_{y}\right) U_{\mathrm{g}}^{(y)}\left(\phi_{x}, \phi_{y}\right)$ of $\phi_{y}$ and with the parameter $\phi_{x}$. The initial condition is $P\left(\phi_{x}, 0\right) U_{\mathrm{g}}^{(y)}\left(\phi_{x}, 0\right)=P\left(\phi_{x}, 0\right)$. By the uniqueness of the solution, $P\left(\phi_{x}, \phi_{y}\right) U_{\mathrm{g}}^{(y)}\left(\phi_{x}, \phi_{y}\right)$ must coincide with $U_{\mathrm{g}}^{(y)}\left(\phi_{x}, \phi_{y}\right) P\left(\phi_{x}, 0\right)$ which has the same initial condition $U_{\mathrm{g}}^{(y)}\left(\phi_{x}, 0\right) P\left(\phi_{x}, 0\right)=P\left(\phi_{x}, 0\right)$. Namely one has

$$
P\left(\phi_{x}, \phi_{y}\right) U_{\mathrm{g}}^{(y)}\left(\phi_{x}, \phi_{y}\right)=U_{\mathrm{g}}^{(y)}\left(\phi_{x}, \phi_{y}\right) P\left(\phi_{x}, 0\right) \text {. }
$$

Combining this with (A.20), one gets

$$
\begin{aligned}
P\left(\phi_{x}, \phi_{y}\right) U_{\mathrm{g}}^{(y)}\left(\phi_{x}, \phi_{y}\right) U_{\mathrm{g}}^{(x)}\left(\phi_{x}\right) & =U_{\mathrm{g}}^{(y)}\left(\phi_{x}, \phi_{y}\right) P\left(\phi_{x}, 0\right) U_{\mathrm{g}}^{(x)}\left(\phi_{x}\right) \\
& =U_{\mathrm{g}}^{(y)}\left(\phi_{x}, \phi_{y}\right) U_{\mathrm{g}}^{(x)}\left(\phi_{x}\right) P(0,0) .
\end{aligned}
$$

Let $U_{\mathrm{g}}(\phi)=U_{\mathrm{g}}^{(y)}(\phi) U_{\mathrm{g}}^{(x)}\left(\phi_{x}\right)$. Then the operator $U_{\mathrm{g}}(\phi)$ is invertible and both the operator $U_{\mathrm{g}}(\phi)$ and its inverse are infinitely differentiable with respect to $\phi$. Besides, the above result is rewritten in the desired form as

$$
P(\phi)=U_{\mathrm{g}}(\phi) P(0,0) U_{\mathrm{g}}^{-1}(\phi) .
$$


Revisiting the Charge Transport in Quantum Hall Systems

\section{B Proof of Proposition 6.2}

In order to prove Proposition 6.2, we need some lemmas.

Lemma B.1 Let $z \notin \sigma(\mathcal{H}(\phi))$. Then

$$
\left\|v_{i}^{(0)}(\boldsymbol{\phi}) R(z ; \boldsymbol{\phi})\right\| \leq \sqrt{\frac{2}{m_{e}}\left[\frac{1}{\operatorname{dist}(z, \sigma(\mathcal{H}(\boldsymbol{\phi})))}+\frac{|z|+\|W\|_{\infty}}{\operatorname{dist}(z, \sigma(\mathcal{H}(\boldsymbol{\phi})))^{2}}\right]}
$$

with the velocity operator $v_{i}^{(0)}(\boldsymbol{\phi})=\left[p_{i}+e A_{i}(\mathbf{r})+\phi_{i}\right] / m_{e}$ for $i=x, y$.

Proof: For any vector $\psi$, one has

$$
\begin{aligned}
\left\|v_{i}^{(0)}(\boldsymbol{\phi}) \frac{1}{z-\mathcal{H}(\boldsymbol{\phi})} \psi\right\|^{2} & =\left\langle\psi, \frac{1}{z^{*}-\mathcal{H}(\boldsymbol{\phi})}\left[v_{i}^{(0)}(\boldsymbol{\phi})\right]^{2} \frac{1}{z-\mathcal{H}(\boldsymbol{\phi})} \psi\right\rangle \\
& \leq \frac{2}{m_{e}}\left\langle\psi, \frac{1}{z^{*}-\mathcal{H}(\boldsymbol{\phi})}\left[\mathcal{H}(\boldsymbol{\phi})+\|W\|_{\infty}\right] \frac{1}{z-\mathcal{H}(\boldsymbol{\phi})} \psi\right\rangle \\
& =\frac{2}{m_{e}} \sum_{n}\left|\left\langle\psi, \psi_{n}\right\rangle\right|^{2} \frac{1}{z^{*}-\mathcal{E}_{n}}\left(\mathcal{E}_{n}+\|W\|_{\infty}\right) \frac{1}{z-\mathcal{E}_{n}} .
\end{aligned}
$$

Here, since

$$
\begin{aligned}
\left|\frac{1}{z^{*}-\mathcal{E}_{n}}\left(\mathcal{E}_{n}+\|W\|_{\infty}\right) \frac{1}{z-\mathcal{E}_{n}}\right| & =\left|\frac{1}{z^{*}-\mathcal{E}_{n}}\left(\mathcal{E}_{n}-z+z+\|W\|_{\infty}\right) \frac{1}{z-\mathcal{E}_{n}}\right| \\
& =\left|-\frac{1}{z^{*}-\mathcal{E}_{n}}+\frac{z+\|W\|_{\infty}}{\left|z-\mathcal{E}_{n}\right|^{2}}\right| \\
& \leq \frac{1}{\operatorname{dist}(z, \sigma(\mathcal{H}(\phi)))}+\frac{|z|+\|W\|_{\infty}}{\operatorname{dist}(z, \sigma(\mathcal{H}(\phi)))^{2}}
\end{aligned}
$$

the desired bound is obtained.

Lemma B.2 Let $z \notin \sigma(\mathcal{H}(\boldsymbol{\phi}+\delta \phi)) \cup \sigma(\mathcal{H}(\boldsymbol{\phi}))$. Then

$$
\|R(z ; \phi+\delta \phi)-R(z ; \phi)\| \leq \mathcal{C} \max _{i=x, y}\left|\delta \phi_{i}\right|
$$

where the positive constant $\mathcal{C}$ depends on $\operatorname{dist}(z, \sigma(\mathcal{H}(\phi+\delta \phi)) \cup \sigma(\mathcal{H}(\phi)))$.

Proof: From

$$
\mathcal{H}(\boldsymbol{\phi}+\delta \boldsymbol{\phi})-\mathcal{H}(\boldsymbol{\phi})=\frac{1}{m_{e}} \delta \boldsymbol{\phi} \cdot[\mathbf{p}+e \mathbf{A}(\mathbf{r})+\boldsymbol{\phi}]+\frac{1}{2 m_{e}}(\delta \boldsymbol{\phi})^{2},
$$

one has

$$
\begin{aligned}
R(z ; \boldsymbol{\phi}+\delta \boldsymbol{\phi})-R(z ; \boldsymbol{\phi}) & =\sum_{i=x, y} R(z ; \boldsymbol{\phi}+\delta \boldsymbol{\phi}) v_{i}^{(0)}(\boldsymbol{\phi}) R(z ; \boldsymbol{\phi}) \delta \phi_{i} \\
& +\frac{1}{2 m_{e}} R(z ; \boldsymbol{\phi}+\delta \boldsymbol{\phi}) R(z ; \boldsymbol{\phi})\left(\delta \phi_{x}^{2}+\delta \phi_{y}^{2}\right) .
\end{aligned}
$$


The norm is evaluated as

$$
\begin{aligned}
\|R(z ; \boldsymbol{\phi}+\delta \boldsymbol{\phi})-R(z ; \boldsymbol{\phi})\| & \leq \sum_{i=x, y}\|R(z ; \boldsymbol{\phi}+\delta \boldsymbol{\phi})\|\left\|v_{i}^{(0)}(\boldsymbol{\phi}) R(z ; \boldsymbol{\phi})\right\|\left|\delta \phi_{i}\right| \\
& +\frac{1}{2 m_{e}}\|R(z ; \boldsymbol{\phi}+\delta \boldsymbol{\phi})\|\|R(z ; \boldsymbol{\phi})\|\left(\delta \phi_{x}^{2}+\delta \phi_{y}^{2}\right) .
\end{aligned}
$$

Consequently, from Lemma B.1, the bound (B.4) is obtained.

Lemma B.3 The following bound is valid:

$$
\left\|Q\left(\mathcal{E} \leq \mathcal{E}_{\mathrm{F}} ; \phi+\delta \phi\right)-Q\left(\mathcal{E} \leq \mathcal{E}_{\mathrm{F}} ; \phi\right)\right\| \leq \mathcal{C} \max _{i=x, y}\left|\delta \phi_{i}\right|
$$

with a positive constant $\mathcal{C}$.

Proof: By definitions,

$$
\begin{aligned}
\left\|Q\left(\mathcal{E} \leq \mathcal{E}_{\mathrm{F}} ; \boldsymbol{\phi}+\delta \boldsymbol{\phi}\right)-Q\left(\mathcal{E} \leq \mathcal{E}_{\mathrm{F}} ; \boldsymbol{\phi}\right)\right\| & =\left\|\frac{1}{2 \pi i} \int_{\gamma}[R(z ; \boldsymbol{\phi}+\delta \boldsymbol{\phi})-R(z ; \boldsymbol{\phi})] d z\right\| \\
& \leq \frac{|\gamma|}{2 \pi} \max _{z \in \gamma}\|R(z ; \boldsymbol{\phi}+\delta \boldsymbol{\phi})-R(z ; \boldsymbol{\phi})\|,
\end{aligned}
$$

where $|\gamma|$ denotes the total length of the path $\gamma$. Therefore the inequality (B.8) follows from Lemma B.2.

Lemma B.4 The following bound is valid:

$$
\left\|Q_{i}\left(\mathcal{E} \leq \mathcal{E}_{\mathrm{F}} ; \phi+\delta \phi\right)-Q_{i}\left(\mathcal{E} \leq \mathcal{E}_{\mathrm{F}} ; \phi\right)\right\| \leq \mathcal{C} \max _{j=x, y}\left|\delta \phi_{j}\right|
$$

with a positive constant $\mathcal{C}$.

Proof: Since

$$
\int_{\gamma}[R(z ; \phi)]^{2} d z=0
$$

one has another expression

$$
Q_{i}\left(\mathcal{E} \leq \mathcal{E}_{\mathrm{F}} ; \boldsymbol{\phi}\right)=\frac{1}{2 \pi i} \int_{\gamma} R(z ; \boldsymbol{\phi}) v_{i}^{(0)}\left(\boldsymbol{\phi}^{\prime}\right) R(z ; \boldsymbol{\phi}) d z \quad \text { with } \quad v_{i}^{(0)}\left(\boldsymbol{\phi}^{\prime}\right)=\frac{1}{m_{e}}\left[p_{i}+e A_{i}(\mathbf{r})+\phi_{i}^{\prime}\right]
$$

for $i=x, y$ and for any $\phi^{\prime}$. Using this expression (B.12), one has

$$
\begin{aligned}
& Q_{i}\left(\mathcal{E} \leq \mathcal{E}_{\mathrm{F}} ; \boldsymbol{\phi}+\delta \boldsymbol{\phi}\right)-Q_{i}\left(\mathcal{E} \leq \mathcal{E}_{\mathrm{F}} ; \boldsymbol{\phi}\right) \\
= & \frac{1}{2 \pi i} \int_{\gamma}[R(z ; \boldsymbol{\phi}+\delta \boldsymbol{\phi})-R(z ; \boldsymbol{\phi})] v_{i}^{(0)}(\boldsymbol{\phi}+\delta \boldsymbol{\phi}) R(z ; \boldsymbol{\phi}+\delta \boldsymbol{\phi}) d z \\
+ & \frac{1}{2 \pi i} \int_{\gamma} R(z ; \boldsymbol{\phi}) v_{i}^{(0)}(\boldsymbol{\phi}+\delta \boldsymbol{\phi})[R(z ; \boldsymbol{\phi}+\delta \boldsymbol{\phi})-R(z ; \boldsymbol{\phi})] d z
\end{aligned}
$$


The norm can be evaluated as

$$
\begin{aligned}
& \left\|Q_{i}\left(\mathcal{E} \leq \mathcal{E}_{\mathrm{F}} ; \boldsymbol{\phi}+\delta \boldsymbol{\phi}\right)-Q_{i}\left(\mathcal{E} \leq \mathcal{E}_{\mathrm{F}} ; \boldsymbol{\phi}\right)\right\| \\
\leq & \frac{|\gamma|}{2 \pi} \max _{z \in \gamma}\|R(z ; \boldsymbol{\phi}+\delta \boldsymbol{\phi})-R(z ; \boldsymbol{\phi})\|\left\|v_{i}^{(0)}(\boldsymbol{\phi}+\delta \boldsymbol{\phi}) R(z ; \boldsymbol{\phi}+\delta \boldsymbol{\phi})\right\| \\
+ & \frac{|\gamma|}{2 \pi} \max _{z \in \gamma}\|R(z ; \boldsymbol{\phi})\|\left\|v_{i}^{(0)}(\boldsymbol{\phi}+\delta \boldsymbol{\phi})[R(z ; \boldsymbol{\phi}+\delta \boldsymbol{\phi})-R(z ; \boldsymbol{\phi})]\right\| .
\end{aligned}
$$

Using Lemmas B.1 and B.2, the first term in the right-hand side can be evaluated, and one can get the desired bound for the first term. Thus it is sufficient to evaluated the second term. Using the identity (B.6), one has

$$
\begin{aligned}
& \left\|v_{i}^{(0)}(\boldsymbol{\phi}+\delta \boldsymbol{\phi})[R(z ; \boldsymbol{\phi}+\delta \boldsymbol{\phi})-R(z ; \boldsymbol{\phi})]\right\| \\
\leq & \sum_{j=x, y}\left\|v_{i}^{(0)}(\boldsymbol{\phi}+\delta \boldsymbol{\phi}) R(z ; \boldsymbol{\phi}+\delta \boldsymbol{\phi})\right\|\left\|v_{j}^{(0)}(\boldsymbol{\phi}) R(z ; \boldsymbol{\phi})\right\|\left|\delta \phi_{j}\right| \\
+ & \frac{1}{2 m_{e}}\left\|v_{i}^{(0)}(\boldsymbol{\phi}+\delta \boldsymbol{\phi}) R(z ; \boldsymbol{\phi}+\delta \boldsymbol{\phi})\right\|\|R(z ; \boldsymbol{\phi})\|\left(\delta \phi_{x}^{2}+\delta \phi_{y}^{2}\right) .
\end{aligned}
$$

Combining this with Lemma B.1, one can get the desired bound also for the second term in the right-hand side of (B.14).

Now we shall give the proof of Proposition 6.2. Since all the cases can be treated in the same way, we treat the case with $i=x, j=y$ only. Note that

$$
\begin{aligned}
& \operatorname{Tr}\left[Q\left(\mathcal{E} \leq \mathcal{E}_{\mathrm{F}} ; \boldsymbol{\phi}+\delta \boldsymbol{\phi}\right) Q_{x}\left(\mathcal{E} \leq \mathcal{E}_{\mathrm{F}} ; \boldsymbol{\phi}+\delta \boldsymbol{\phi}\right) Q_{y}\left(\mathcal{E} \leq \mathcal{E}_{\mathrm{F}} ; \boldsymbol{\phi}+\delta \boldsymbol{\phi}\right)\right] \\
& -\operatorname{Tr}\left[Q\left(\mathcal{E} \leq \mathcal{E}_{\mathrm{F}} ; \boldsymbol{\phi}\right) Q_{x}\left(\mathcal{E} \leq \mathcal{E}_{\mathrm{F}} ; \boldsymbol{\phi}\right) Q_{y}\left(\mathcal{E} \leq \mathcal{E}_{\mathrm{F}} ; \boldsymbol{\phi}\right)\right] \\
= & \operatorname{Tr}\left[\left\{Q\left(\mathcal{E} \leq \mathcal{E}_{\mathrm{F}} ; \boldsymbol{\phi}+\delta \boldsymbol{\phi}\right)-Q\left(\mathcal{E} \leq \mathcal{E}_{\mathrm{F}} ; \boldsymbol{\phi}\right)\right\} Q_{x}\left(\mathcal{E} \leq \mathcal{E}_{\mathrm{F}} ; \boldsymbol{\phi}+\delta \boldsymbol{\phi}\right) Q_{y}\left(\mathcal{E} \leq \mathcal{E}_{\mathrm{F}} ; \boldsymbol{\phi}+\delta \boldsymbol{\phi}\right)\right] \\
+ & \operatorname{Tr}\left[Q\left(\mathcal{E} \leq \mathcal{E}_{\mathrm{F}} ; \boldsymbol{\phi}\right)\left\{Q_{x}\left(\mathcal{E} \leq \mathcal{E}_{\mathrm{F}} ; \boldsymbol{\phi}+\delta \boldsymbol{\phi}\right)-Q_{x}\left(\mathcal{E} \leq \mathcal{E}_{\mathrm{F}} ; \boldsymbol{\phi}\right)\right\} Q_{y}\left(\mathcal{E} \leq \mathcal{E}_{\mathrm{F}} ; \boldsymbol{\phi}+\delta \boldsymbol{\phi}\right)\right] \\
+ & \operatorname{Tr}\left[Q\left(\mathcal{E} \leq \mathcal{E}_{\mathrm{F}} ; \boldsymbol{\phi}\right) Q_{x}\left(\mathcal{E} \leq \mathcal{E}_{\mathrm{F}} ; \boldsymbol{\phi}\right)\left\{Q_{y}\left(\mathcal{E} \leq \mathcal{E}_{\mathrm{F}} ; \boldsymbol{\phi}+\delta \boldsymbol{\phi}\right)-Q_{y}\left(\mathcal{E} \leq \mathcal{E}_{\mathrm{F}} ; \boldsymbol{\phi}\right)\right\}\right]
\end{aligned}
$$

Consider first the first term in the right-hand side. Using the identity,

$$
Q_{i}\left(\mathcal{E} \leq \mathcal{E}_{\mathrm{F}} ; \phi\right)=Q\left(\mathcal{E} \leq \mathcal{E}_{\mathrm{F}} ; \phi\right) Q_{i}\left(\mathcal{E} \leq \mathcal{E}_{\mathrm{F}} ; \phi\right)+Q_{i}\left(\mathcal{E} \leq \mathcal{E}_{\mathrm{F}} ; \phi\right) Q\left(\mathcal{E} \leq \mathcal{E}_{\mathrm{F}} ; \phi\right)
$$

which is the non-interacting version of (5.9), one has

$$
\begin{aligned}
& \operatorname{Tr}\left[\delta Q_{\leq} Q_{x}\left(\mathcal{E} \leq \mathcal{E}_{\mathrm{F}} ; \boldsymbol{\phi}+\delta \boldsymbol{\phi}\right) Q_{y}\left(\mathcal{E} \leq \mathcal{E}_{\mathrm{F}} ; \boldsymbol{\phi}+\delta \boldsymbol{\phi}\right)\right] \\
= & \sum_{m: \mathcal{E}_{m} \leq \mathcal{E}_{\mathrm{F}}}\left\langle\tilde{\psi}_{m}, Q_{x}\left(\mathcal{E} \leq \mathcal{E}_{\mathrm{F}} ; \boldsymbol{\phi}+\delta \boldsymbol{\phi}\right) Q_{y}\left(\mathcal{E} \leq \mathcal{E}_{\mathrm{F}} ; \boldsymbol{\phi}+\delta \boldsymbol{\phi}\right) \delta Q_{\leq} \tilde{\psi}_{m}\right\rangle \\
+ & \sum_{m: \mathcal{E}_{m} \leq \mathcal{E}_{\mathrm{F}}}\left\langle\tilde{\psi}_{m}, Q_{y}\left(\mathcal{E} \leq \mathcal{E}_{\mathrm{F}} ; \boldsymbol{\phi}+\delta \boldsymbol{\phi}\right) \delta Q_{\leq} Q_{x}\left(\mathcal{E} \leq \mathcal{E}_{\mathrm{F}} ; \boldsymbol{\phi}+\delta \boldsymbol{\phi}\right) \tilde{\psi}_{m}\right\rangle,
\end{aligned}
$$

where the vectors $\tilde{\psi}_{m}$ are the energy eigenvectors of the single electron Hamiltonian with $\boldsymbol{\phi}+\delta \boldsymbol{\phi}$, and we have written $\delta Q_{\leq}=Q\left(\mathcal{E} \leq \mathcal{E}_{\mathrm{F}} ; \boldsymbol{\phi}+\delta \boldsymbol{\phi}\right)-Q\left(\mathcal{E} \leq \mathcal{E}_{\mathrm{F}} ; \boldsymbol{\phi}\right)$. Immediately,

$$
\begin{aligned}
& \left|\operatorname{Tr}\left[\delta Q_{\leq} Q_{x}\left(\mathcal{E} \leq \mathcal{E}_{\mathrm{F}} ; \boldsymbol{\phi}+\delta \boldsymbol{\phi}\right) Q_{y}\left(\mathcal{E} \leq \mathcal{E}_{\mathrm{F}} ; \boldsymbol{\phi}+\delta \boldsymbol{\phi}\right)\right]\right| \\
\leq & 2 N\left\|Q_{x}\left(\mathcal{E} \leq \mathcal{E}_{\mathrm{F}} ; \boldsymbol{\phi}+\delta \boldsymbol{\phi}\right)\right\|\left\|Q_{y}\left(\mathcal{E} \leq \mathcal{E}_{\mathrm{F}} ; \boldsymbol{\phi}+\delta \boldsymbol{\phi}\right)\right\|\left\|\delta Q_{\leq}\right\|,
\end{aligned}
$$


where $N$ is the number of the electrons. Here the operators $Q_{i}\left(\mathcal{E} \leq \mathcal{E}_{\mathrm{F}} ; \boldsymbol{\phi}+\delta \boldsymbol{\phi}\right)$ are bounded because of Lemma B.1 and (B.12). Combining these observations with Lemma B.3, one gets

$$
\begin{aligned}
& \left|\operatorname{Tr}\left[\left\{Q\left(\mathcal{E} \leq \mathcal{E}_{\mathrm{F}} ; \phi+\delta \phi\right)-Q\left(\mathcal{E} \leq \mathcal{E}_{\mathrm{F}} ; \phi\right)\right\} Q_{x}\left(\mathcal{E} \leq \mathcal{E}_{\mathrm{F}} ; \boldsymbol{\phi}+\delta \phi\right) Q_{y}\left(\mathcal{E} \leq \mathcal{E}_{\mathrm{F}} ; \phi+\delta \phi\right)\right]\right| \\
\leq & \mathcal{C} N \max _{i=x, y}\left|\delta \phi_{i}\right|
\end{aligned}
$$

with a positive constant $\mathcal{C}$. In a similar way, one has

$$
\begin{aligned}
& \left|\operatorname{Tr}\left[Q\left(\mathcal{E} \leq \mathcal{E}_{\mathrm{F}} ; \boldsymbol{\phi}\right)\left\{Q_{x}\left(\mathcal{E} \leq \mathcal{E}_{\mathrm{F}} ; \boldsymbol{\phi}+\delta \boldsymbol{\phi}\right)-Q_{x}\left(\mathcal{E} \leq \mathcal{E}_{\mathrm{F}} ; \boldsymbol{\phi}\right)\right\} Q_{y}\left(\mathcal{E} \leq \mathcal{E}_{\mathrm{F}} ; \boldsymbol{\phi}+\delta \boldsymbol{\phi}\right)\right]\right| \\
\leq & \sum_{m: \mathcal{E}_{m} \leq \mathcal{E}_{\mathrm{F}}}\left|\left\langle\psi_{m},\left\{Q_{x}\left(\mathcal{E} \leq \mathcal{E}_{\mathrm{F}} ; \boldsymbol{\phi}+\delta \boldsymbol{\phi}\right)-Q_{x}\left(\mathcal{E} \leq \mathcal{E}_{\mathrm{F}} ; \boldsymbol{\phi}\right)\right\} Q_{y}\left(\mathcal{E} \leq \mathcal{E}_{\mathrm{F}} ; \boldsymbol{\phi}+\delta \boldsymbol{\phi}\right) \psi_{m}\right\rangle\right| \\
\leq & N\left\|Q_{x}\left(\mathcal{E} \leq \mathcal{E}_{\mathrm{F}} ; \boldsymbol{\phi}+\delta \boldsymbol{\phi}\right)-Q_{x}\left(\mathcal{E} \leq \mathcal{E}_{\mathrm{F}} ; \boldsymbol{\phi}\right)\right\|\left\|Q_{y}\left(\mathcal{E} \leq \mathcal{E}_{\mathrm{F}} ; \boldsymbol{\phi}+\delta \boldsymbol{\phi}\right)\right\| \\
\leq & \mathcal{C} N \max _{i=x, y}\left|\delta \phi_{i}\right|,
\end{aligned}
$$

where the vectors $\psi_{m}$ are the energy eigenvectors of the single electron Hamiltonian with $\phi$, and $\mathcal{C}$ is a positive constant, and we have used Lemma B.4 for getting the last inequality. In the same way,

$$
\begin{aligned}
& \left|\operatorname{Tr}\left[Q\left(\mathcal{E} \leq \mathcal{E}_{\mathrm{F}} ; \phi\right) Q_{x}\left(\mathcal{E} \leq \mathcal{E}_{\mathrm{F}} ; \phi\right)\left\{Q_{y}\left(\mathcal{E} \leq \mathcal{E}_{\mathrm{F}} ; \phi+\delta \phi\right)-Q_{y}\left(\mathcal{E} \leq \mathcal{E}_{\mathrm{F}} ; \phi\right)\right\}\right]\right| \\
\leq & \mathcal{C} N \max _{i=x, y}\left|\delta \phi_{i}\right|
\end{aligned}
$$

with a positive constant $\mathcal{C}$. Combining these three inequalities with (B.16), one can obtain the desired bound (6.35) in the case with $i=x, j=y$.

\section{Proof of Proposition 6.4}

Since $\operatorname{Tr} Q_{y}\left(\mathcal{E} \leq \mathcal{E}_{F} ; \phi\right)=0$, it is sufficient to show

$$
\left|\operatorname{Tr} v_{s}^{(0)}(\boldsymbol{\phi}+\delta \boldsymbol{\phi}) Q_{y}\left(\mathcal{E} \leq \mathcal{E}_{F} ; \boldsymbol{\phi}+\delta \boldsymbol{\phi}\right)-\operatorname{Tr} v_{s}^{(0)}(\boldsymbol{\phi}+\delta \boldsymbol{\phi}) Q_{y}\left(\mathcal{E} \leq \mathcal{E}_{F} ; \boldsymbol{\phi}\right)\right| \leq \mathcal{C} N \max _{i=x, y}\left|\delta \phi_{i}\right|
$$

with some positive constant $\mathcal{C}$. Using the identity (B.17), one has

$$
\begin{gathered}
\operatorname{Tr} v_{s}^{(0)}(\boldsymbol{\phi}+\delta \boldsymbol{\phi}) Q_{y}\left(\mathcal{E} \leq \mathcal{E}_{F} ; \boldsymbol{\phi}+\delta \boldsymbol{\phi}\right)-\operatorname{Tr} v_{s}^{(0)}(\boldsymbol{\phi}+\delta \boldsymbol{\phi}) Q_{y}\left(\mathcal{E} \leq \mathcal{E}_{F} ; \boldsymbol{\phi}\right) \\
=\operatorname{Tr} v_{s}^{(0)}(\boldsymbol{\phi}+\delta \boldsymbol{\phi})\left\{Q_{y}\left(\mathcal{E} \leq \mathcal{E}_{F} ; \boldsymbol{\phi}+\delta \boldsymbol{\phi}\right) Q\left(\mathcal{E} \leq \mathcal{E}_{F} ; \boldsymbol{\phi}+\delta \boldsymbol{\phi}\right)\right. \\
\left.\quad-Q_{y}\left(\mathcal{E} \leq \mathcal{E}_{F} ; \boldsymbol{\phi}\right) Q\left(\mathcal{E} \leq \mathcal{E}_{F} ; \boldsymbol{\phi}\right)\right\} \\
+\operatorname{Tr} v_{s}^{(0)}(\boldsymbol{\phi}+\delta \boldsymbol{\phi})\left\{Q\left(\mathcal{E} \leq \mathcal{E}_{F} ; \boldsymbol{\phi}+\delta \boldsymbol{\phi}\right) Q_{y}\left(\mathcal{E} \leq \mathcal{E}_{F} ; \boldsymbol{\phi}+\delta \boldsymbol{\phi}\right)\right. \\
\left.\quad-Q\left(\mathcal{E} \leq \mathcal{E}_{F} ; \boldsymbol{\phi}\right) Q_{y}\left(\mathcal{E} \leq \mathcal{E}_{F} ; \boldsymbol{\phi}\right)\right\} .
\end{gathered}
$$

We begin with estimating the first term in the right-hand side. Note that

[1st term in r.h.s. of (C.2)]

$$
\begin{aligned}
& =\operatorname{Tr} v_{s}^{(0)}(\boldsymbol{\phi}+\delta \boldsymbol{\phi})\left\{Q_{y}\left(\mathcal{E} \leq \mathcal{E}_{F} ; \boldsymbol{\phi}+\delta \boldsymbol{\phi}\right)-Q_{y}\left(\mathcal{E} \leq \mathcal{E}_{F} ; \boldsymbol{\phi}\right)\right\} Q\left(\mathcal{E} \leq \mathcal{E}_{F} ; \boldsymbol{\phi}+\delta \boldsymbol{\phi}\right) \\
& +\operatorname{Tr} v_{s}^{(0)}(\boldsymbol{\phi}+\delta \boldsymbol{\phi}) Q_{y}\left(\mathcal{E} \leq \mathcal{E}_{F} ; \boldsymbol{\phi}\right) Q\left(\mathcal{E} \leq \mathcal{E}_{F} ; \boldsymbol{\phi}\right)\left\{Q\left(\mathcal{E} \leq \mathcal{E}_{F} ; \boldsymbol{\phi}+\delta \boldsymbol{\phi}\right)-Q\left(\mathcal{E} \leq \mathcal{E}_{F} ; \boldsymbol{\phi}\right)\right\} \\
& +\operatorname{Tr} v_{s}^{(0)}(\boldsymbol{\phi}+\delta \boldsymbol{\phi}) Q\left(\mathcal{E} \leq \mathcal{E}_{F} ; \boldsymbol{\phi}\right) Q_{y}\left(\mathcal{E} \leq \mathcal{E}_{F} ; \boldsymbol{\phi}\right)\left\{Q\left(\mathcal{E} \leq \mathcal{E}_{F} ; \boldsymbol{\phi}+\delta \boldsymbol{\phi}\right)-Q\left(\mathcal{E} \leq \mathcal{E}_{F} ; \boldsymbol{\phi}\right)\right\},
\end{aligned}
$$


where we have used the identity (B.17) again. This first term in the right-hand side is rewritten as

$$
\begin{aligned}
& \operatorname{Tr} v_{s}^{(0)}(\boldsymbol{\phi}+\delta \boldsymbol{\phi})\left\{Q_{y}\left(\mathcal{E} \leq \mathcal{E}_{F} ; \boldsymbol{\phi}+\delta \boldsymbol{\phi}\right)-Q_{y}\left(\mathcal{E} \leq \mathcal{E}_{F} ; \boldsymbol{\phi}\right)\right\} Q\left(\mathcal{E} \leq \mathcal{E}_{F} ; \boldsymbol{\phi}+\delta \boldsymbol{\phi}\right) \\
= & \sum_{m: \mathcal{E}_{m} \leq \mathcal{E}_{F}}\left\langle\tilde{\psi}_{m}, v_{s}^{(0)}(\boldsymbol{\phi}+\delta \boldsymbol{\phi})\left\{Q_{y}\left(\mathcal{E} \leq \mathcal{E}_{F} ; \boldsymbol{\phi}+\delta \boldsymbol{\phi}\right)-Q_{y}\left(\mathcal{E} \leq \mathcal{E}_{F} ; \boldsymbol{\phi}\right)\right\} \tilde{\psi}_{m}\right\rangle
\end{aligned}
$$

where the vectors $\tilde{\psi}_{m}$ are the energy eigenvectors of the single electron Hamiltonian with the gauge parameters $\phi+\delta \phi$. From Lemma B.1, (B.13) and (B.15), one has

$$
\left\|v_{s}^{(0)}(\boldsymbol{\phi}+\delta \boldsymbol{\phi})\left\{Q_{y}\left(\mathcal{E} \leq \mathcal{E}_{F} ; \boldsymbol{\phi}+\delta \boldsymbol{\phi}\right)-Q_{y}\left(\mathcal{E} \leq \mathcal{E}_{F} ; \boldsymbol{\phi}\right)\right\}\right\| \leq \mathcal{C} \max _{i=x, y}\left|\delta \phi_{i}\right|,
$$

where the positive constant $\mathcal{C}$ is independent of the number $N$ of the electrons and the system sizes $L_{x}, L_{y}$. Using this bound for (C.4), one obtains

$$
\begin{aligned}
& \left|\operatorname{Tr} v_{s}^{(0)}(\boldsymbol{\phi}+\delta \boldsymbol{\phi})\left\{Q_{y}\left(\mathcal{E} \leq \mathcal{E}_{F} ; \boldsymbol{\phi}+\delta \boldsymbol{\phi}\right)-Q_{y}\left(\mathcal{E} \leq \mathcal{E}_{F} ; \boldsymbol{\phi}\right)\right\} Q\left(\mathcal{E} \leq \mathcal{E}_{F} ; \boldsymbol{\phi}+\delta \boldsymbol{\phi}\right)\right| \\
\leq & \mathcal{C} N \max _{i=x, y}\left|\delta \phi_{i}\right| .
\end{aligned}
$$

The second term in the right-hand side of (C.3) is rewritten as

$$
\begin{aligned}
& \operatorname{Tr} v_{s}^{(0)}(\boldsymbol{\phi}+\delta \boldsymbol{\phi}) Q_{y}\left(\mathcal{E} \leq \mathcal{E}_{F} ; \boldsymbol{\phi}\right) Q\left(\mathcal{E} \leq \mathcal{E}_{F} ; \boldsymbol{\phi}\right)\left\{Q\left(\mathcal{E} \leq \mathcal{E}_{F} ; \boldsymbol{\phi}+\delta \boldsymbol{\phi}\right)-Q\left(\mathcal{E} \leq \mathcal{E}_{F} ; \boldsymbol{\phi}\right)\right\} \\
= & \sum_{m: \mathcal{E}_{m} \leq \mathcal{E}_{F}}\left\langle\psi_{m},\left\{Q\left(\mathcal{E} \leq \mathcal{E}_{F} ; \boldsymbol{\phi}+\delta \boldsymbol{\phi}\right)-Q\left(\mathcal{E} \leq \mathcal{E}_{F} ; \boldsymbol{\phi}\right)\right\} v_{s}^{(0)}(\boldsymbol{\phi}+\delta \boldsymbol{\phi}) Q_{y}\left(\mathcal{E} \leq \mathcal{E}_{F} ; \boldsymbol{\phi}\right) \psi_{m}\right\rangle,
\end{aligned}
$$

where $\psi_{m}$ are the energy eigenvectors of the single electron Hamiltonian with the gauge parameters $\boldsymbol{\phi}$. From Lemma B.1, the operator $v_{s}^{(0)}(\boldsymbol{\phi}+\delta \phi) Q_{y}\left(\mathcal{E} \leq \mathcal{E}_{F} ; \boldsymbol{\phi}\right)$ is bounded, and the difference $Q\left(\mathcal{E} \leq \mathcal{E}_{F} ; \phi+\delta \phi\right)-Q\left(\mathcal{E} \leq \mathcal{E}_{F} ; \phi\right)$ was already estimated in Lemma B.3. Therefore

$$
\begin{aligned}
& \left|\operatorname{Tr} v_{s}^{(0)}(\boldsymbol{\phi}+\delta \boldsymbol{\phi}) Q_{y}\left(\mathcal{E} \leq \mathcal{E}_{F} ; \boldsymbol{\phi}\right) Q\left(\mathcal{E} \leq \mathcal{E}_{F} ; \boldsymbol{\phi}\right)\left\{Q\left(\mathcal{E} \leq \mathcal{E}_{F} ; \boldsymbol{\phi}+\delta \boldsymbol{\phi}\right)-Q\left(\mathcal{E} \leq \mathcal{E}_{F} ; \boldsymbol{\phi}\right)\right\}\right| \\
\leq & \mathcal{C} N \max _{i=x, y}\left|\delta \phi_{i}\right|,
\end{aligned}
$$

where the positive constant $\mathcal{C}$ is independent of the number $N$ of the electrons and the system sizes $L_{x}, L_{y}$. Similarly the third term in the right-hand side of (C.3) is evaluated as

$$
\begin{aligned}
& \left|\operatorname{Tr} v_{s}^{(0)}(\boldsymbol{\phi}+\delta \boldsymbol{\phi}) Q\left(\mathcal{E} \leq \mathcal{E}_{F} ; \boldsymbol{\phi}\right) Q_{y}\left(\mathcal{E} \leq \mathcal{E}_{F} ; \boldsymbol{\phi}\right)\left\{Q\left(\mathcal{E} \leq \mathcal{E}_{F} ; \boldsymbol{\phi}+\delta \boldsymbol{\phi}\right)-Q\left(\mathcal{E} \leq \mathcal{E}_{F} ; \boldsymbol{\phi}\right)\right\}\right| \\
\leq & \sum_{\mathcal{E}_{m} \leq \mathcal{E}_{F}}\left|\left\langle\psi_{m}, Q_{y}\left(\mathcal{E} \leq \mathcal{E}_{F} ; \boldsymbol{\phi}\right)\left\{Q\left(\mathcal{E} \leq \mathcal{E}_{F} ; \boldsymbol{\phi}+\delta \boldsymbol{\phi}\right)-Q\left(\mathcal{E} \leq \mathcal{E}_{F} ; \boldsymbol{\phi}\right)\right\} v_{s}^{(0)}(\boldsymbol{\phi}+\delta \boldsymbol{\phi}) \psi_{m}\right\rangle\right| \\
\leq & \left\|Q_{y}\left(\mathcal{E} \leq \mathcal{E}_{F} ; \boldsymbol{\phi}\right)\right\|\left\|Q\left(\mathcal{E} \leq \mathcal{E}_{F} ; \boldsymbol{\phi}+\delta \boldsymbol{\phi}\right)-Q\left(\mathcal{E} \leq \mathcal{E}_{F} ; \boldsymbol{\phi}\right)\right\| \\
\times & \left\{\frac{N}{m_{e}}\left|\delta \phi_{s}\right|+\sum_{m: \mathcal{E}_{m} \leq \mathcal{E}_{F}} \sqrt{\left\langle\psi_{m},\left[v_{s}^{(0)}(\boldsymbol{\phi})\right]^{2} \psi_{m}\right\rangle}\right\} \\
\leq & \mathcal{C} \max _{i=x, y}\left|\delta \phi_{i}\right|\left\{\frac{N}{m_{e}}\left|\delta \phi_{s}\right|+N \sqrt{\frac{2}{m_{e}}\left(\mathcal{E}_{F}+\|W\|_{\infty}\right)}\right\} .
\end{aligned}
$$


Thus all of the terms in the right-hand side of (C.3) have been evaluated.

Next consider the second term in the right-hand side of (C.2). It can be rewritten as

$$
\begin{aligned}
& {[\text { 2nd term in r.h.s. of }(\mathrm{C} .2)] } \\
= & \operatorname{Tr} v_{s}^{(0)}(\boldsymbol{\phi}+\delta \boldsymbol{\phi}) \delta Q_{\leq} Q_{y}\left(\mathcal{E} \leq \mathcal{E}_{F} ; \boldsymbol{\phi}+\delta \boldsymbol{\phi}\right) Q\left(\mathcal{E} \leq \mathcal{E}_{F} ; \boldsymbol{\phi}+\delta \boldsymbol{\phi}\right) \\
+ & \operatorname{Tr} v_{s}^{(0)}(\boldsymbol{\phi}+\delta \boldsymbol{\phi}) \delta Q_{\leq} Q\left(\mathcal{E} \leq \mathcal{E}_{F} ; \boldsymbol{\phi}+\delta \boldsymbol{\phi}\right) Q_{y}\left(\mathcal{E} \leq \mathcal{E}_{F} ; \boldsymbol{\phi}+\delta \boldsymbol{\phi}\right) \\
+ & \operatorname{Tr} v_{s}^{(0)}(\boldsymbol{\phi}+\delta \boldsymbol{\phi}) Q\left(\mathcal{E} \leq \mathcal{E}_{F} ; \boldsymbol{\phi}\right)\left\{Q_{y}\left(\mathcal{E} \leq \mathcal{E}_{F} ; \boldsymbol{\phi}+\delta \boldsymbol{\phi}\right)-Q_{y}\left(\mathcal{E} \leq \mathcal{E}_{F} ; \boldsymbol{\phi}\right)\right\}
\end{aligned}
$$

where we have used the identity (B.17), and we have written

$$
\delta Q_{\leq}=Q\left(\mathcal{E} \leq \mathcal{E}_{F} ; \phi+\delta \phi\right)-Q\left(\mathcal{E} \leq \mathcal{E}_{F} ; \phi\right) .
$$

From Lemma B.1 and (B.6), one has

$$
\left\|v_{s}^{(0)}(\boldsymbol{\phi}+\delta \boldsymbol{\phi}) \delta Q_{\leq}\right\| \leq \mathcal{C} \max _{i=x, y}\left|\delta \phi_{i}\right|
$$

where the positive constant $\mathcal{C}$ is independent of the number $N$ of the electrons and the system sizes $L_{x}, L_{y}$. Hence, in the same way as in the above, the first and the second terms in the right-hand side of (C.10) are evaluated as

$$
\begin{aligned}
& \left|\operatorname{Tr} v_{s}^{(0)}(\boldsymbol{\phi}+\delta \boldsymbol{\phi}) \delta Q_{\leq} Q_{y}\left(\mathcal{E} \leq \mathcal{E}_{F} ; \boldsymbol{\phi}+\delta \boldsymbol{\phi}\right) Q\left(\mathcal{E} \leq \mathcal{E}_{F} ; \boldsymbol{\phi}+\delta \boldsymbol{\phi}\right)\right| \\
+ & \left|\operatorname{Tr} v_{s}^{(0)}(\boldsymbol{\phi}+\delta \boldsymbol{\phi}) \delta Q_{\leq} Q\left(\mathcal{E} \leq \mathcal{E}_{F} ; \boldsymbol{\phi}+\delta \boldsymbol{\phi}\right) Q_{y}\left(\mathcal{E} \leq \mathcal{E}_{F} ; \boldsymbol{\phi}+\delta \boldsymbol{\phi}\right)\right| \leq \mathcal{C} N \max _{i=x, y}\left|\delta \phi_{i}\right|
\end{aligned}
$$

with some positive constant $\mathcal{C}$. By using Lemma B.4, the third term in the right-hand side of (C.10) is evaluated as

$$
\left|\operatorname{Tr} v_{s}^{(0)}(\phi+\delta \phi) Q\left(\mathcal{E} \leq \mathcal{E}_{F} ; \phi\right)\left\{Q_{y}\left(\mathcal{E} \leq \mathcal{E}_{F} ; \phi+\delta \phi\right)-Q_{y}\left(\mathcal{E} \leq \mathcal{E}_{F} ; \phi\right)\right\}\right| \leq \mathcal{C} N \max _{i=x, y}\left|\delta \phi_{i}\right|
$$

in the same way as in (C.9).

Combining (C.2), (C.3), (C.6), (C.8), (C.9), (C.10), (C.13) and (C.14), the desired bound (C.1) is obtained.

\section{Proofs of Theorems 7.2 and 7.5}

In order to give the proofs of Theorems 7.2 and 7.5, we first recall the Hall conductance $\sigma_{x y}(\phi)$ of (3.37) which can be expressed as

$$
\sigma_{x y}(\phi)=\frac{i \hbar e^{2}}{L_{x} L_{y}} \frac{1}{q} \sum_{\mu=1}^{q}\left[\left\langle\Phi_{0, \mu}^{(N)}(\phi), v_{\mathrm{tot}, y}^{(0)}(\phi) \frac{1-Q\left(E_{0}^{(N)}(\phi)\right)}{\left[E_{0, \mu}^{(N)}(\phi)-H_{0}^{(N)}(\boldsymbol{\phi})\right]^{2}} v_{\mathrm{tot}, x}^{(0)}(\boldsymbol{\phi}) \Phi_{0, \mu}^{(N)}(\boldsymbol{\phi})\right\rangle-\text { c.c. }\right] .
$$


Using the Schwarz inequality, the matrix element is estimated as

$$
\begin{aligned}
& \left|\sum_{\mu=1}^{q}\left\langle\Phi_{0, \mu}^{(N)}(\phi), v_{\mathrm{tot}, y}^{(0)}(\phi) \frac{1-Q\left(E_{0}^{(N)}(\phi)\right)}{\left[E_{0, \mu}^{(N)}(\phi)-H_{0}^{(N)}(\phi)\right]^{2}} v_{\mathrm{tot}, x}^{(0)}(\phi) \Phi_{0, \mu}^{(N)}(\phi)\right\rangle\right| \\
\leq & \frac{1}{(\Delta E)^{2}} \prod_{s=x, y} \sqrt{\sum_{\mu=1}^{q}\left\langle\Phi_{0, \mu}^{(N)}(\phi), v_{\mathrm{tot}, s}^{(0)}(\phi)\left[1-Q\left(E_{0}^{(N)}(\phi)\right)\right] v_{\mathrm{tot}, s}^{(0)}(\phi) \Phi_{0, \mu}^{(N)}(\phi)\right\rangle .}
\end{aligned}
$$

Because of the factor $1 /\left(L_{x} L_{y}\right)$ in the right-hand side of (D.1), it is sufficient to show that this right-hand side is of order of $N$. But a simple estimate yields order $N^{2}$ because of the two total velocity operators in the matrix elements. In order to reduce the order $N^{2}$ to $N$, we use the method introduced in ref. [51]. See also refs. [31, 52]. Let $A$ be a symmetric operator. In the same way as in (4.23), one has formally

$$
\begin{aligned}
& \sum_{\mu=1}^{q}\left\langle A \Phi_{0, \mu}^{(N)}(\phi),\left[1-Q\left(E_{0}^{(N)}(\phi)\right] A \Phi_{0, \mu}^{(N)}(\phi)\right\rangle\right. \\
\leq & \frac{1}{\Delta E} \sum_{\mu=1}^{q}\left\langle A \Phi_{0, \mu}^{(N)}(\phi),\left[H_{0}^{(N)}(\phi)-E_{0, \mu}^{(N)}(\phi)\right] A \Phi_{0, \mu}^{(N)}(\phi)\right\rangle \\
= & \frac{1}{2 \Delta E} \sum_{\mu=1}^{q}\left\langle\Phi_{0, \mu}^{(N)}(\phi),\left[A,\left[H_{0}^{(N)}(\phi), A\right]\right] \Phi_{0, \mu}^{(N)}(\phi)\right\rangle .
\end{aligned}
$$

We stress that this formal inequality can be justified in the same way as in Section 4 . From the two inequalities (D.2) and (D.3), it is sufficient to show that the expectation values of the double commutators $\left[v_{\mathrm{tot}, s}^{(0)}(\phi),\left[H_{0}^{(N)}(\phi), v_{\mathrm{tot}, s}^{(0)}(\phi)\right]\right]$ for the ground state are of order of $N$.

Let us calculate those double commutators. Note that

$$
\left[v_{\text {tot }, x}^{(0)}(\phi), H_{0}^{(N)}(\boldsymbol{\phi})\right]=-\frac{i \hbar e B}{m_{e}} v_{\text {tot }, y}^{(0)}(\phi)-\frac{i \hbar e B}{m_{e}} I_{y}(\phi),
$$

and

$$
\left[v_{\mathrm{tot}, y}^{(0)}(\boldsymbol{\phi}), H_{0}^{(N)}(\boldsymbol{\phi})\right]=\frac{i \hbar e B}{m_{e}} v_{\mathrm{tot}, x}^{(0)}(\boldsymbol{\phi})-\frac{i \hbar e B}{m_{e}} I_{x}(\boldsymbol{\phi}),
$$

where

$$
I_{x}(\boldsymbol{\phi})=\frac{1}{e B} \sum_{j=1}^{N}\left(\frac{\partial W}{\partial y}\right)\left(\mathbf{r}_{j}\right)-\frac{1}{2 B} \sum_{j=1}^{N}\left[B_{\mathrm{P}, z}\left(\mathbf{r}_{j}\right) v_{x, j}^{(0)}(\boldsymbol{\phi})+v_{x, j}^{(0)}(\boldsymbol{\phi}) B_{\mathrm{P}, z}\left(\mathbf{r}_{j}\right)\right]
$$

and

$$
I_{y}(\boldsymbol{\phi})=\frac{1}{e B} \sum_{j=1}^{N}\left(\frac{\partial W}{\partial x}\right)\left(\mathbf{r}_{j}\right)+\frac{1}{2 B} \sum_{j=1}^{N}\left[B_{\mathrm{P}, z}\left(\mathbf{r}_{j}\right) v_{y, j}^{(0)}(\boldsymbol{\phi})+v_{y, j}^{(0)}(\boldsymbol{\phi}) B_{\mathrm{P}, z}\left(\mathbf{r}_{j}\right)\right]
$$

with the velocity operator,

$$
\mathbf{v}_{j}^{(0)}(\phi)=\frac{1}{m_{e}}\left[\mathbf{p}_{j}+e \mathbf{A}\left(\mathbf{r}_{j}\right)+\phi\right] .
$$


Using the commutation relation (D.4), we have

$$
\left[v_{\mathrm{tot}, x}^{(0)}(\boldsymbol{\phi}),\left[H_{0}^{(N)}(\boldsymbol{\phi}), v_{\mathrm{tot}, x}^{(0)}(\boldsymbol{\phi})\right]\right]=\frac{i \hbar e B}{m_{e}}\left[v_{\mathrm{tot}, x}^{(0)}(\boldsymbol{\phi}), v_{\mathrm{tot}, y}^{(0)}(\boldsymbol{\phi})\right]+\frac{i \hbar e B}{m_{e}}\left[v_{\mathrm{tot}, x}^{(0)}(\phi), I_{y}(\boldsymbol{\phi})\right] .
$$

The commutator of the first term in the right-hand side is calculated as

$$
\left[v_{\mathrm{tot}, x}^{(0)}(\boldsymbol{\phi}), v_{\mathrm{tot}, y}^{(0)}(\boldsymbol{\phi})\right]=-\frac{i \hbar e B}{m_{e}^{2}} N-\frac{i \hbar e}{m_{e}^{2}} \sum_{j=1}^{N} B_{\mathrm{P}, z}\left(\mathbf{r}_{j}\right),
$$

and the commutator of the second term becomes

$$
\begin{aligned}
{\left[v_{\text {tot }, x}^{(0)}(\boldsymbol{\phi}), I_{y}(\boldsymbol{\phi})\right] } & =-\frac{i \hbar}{m_{e} e B} \sum_{j=1}^{N}\left(\frac{\partial^{2} W}{\partial x^{2}}\right)\left(\mathbf{r}_{j}\right)-\frac{i \hbar e}{m_{e}^{2} B} \sum_{j=1}^{N}\left[B_{\mathrm{P}, z}\left(\mathbf{r}_{j}\right)\right]^{2}-\frac{i \hbar e}{m_{e}^{2}} \sum_{j=1}^{N} B_{\mathrm{P}, z}\left(\mathbf{r}_{j}\right) \\
& -\frac{i \hbar}{2 m_{e} B} \sum_{j=1}^{N}\left[v_{y, j}^{(0)}(\boldsymbol{\phi})\left(\frac{\partial B_{\mathrm{P}, z}}{\partial x}\right)\left(\mathbf{r}_{j}\right)+\left(\frac{\partial B_{\mathrm{P}, z}}{\partial x}\right)\left(\mathbf{r}_{j}\right) v_{y, j}^{(0)}(\boldsymbol{\phi})\right] . \quad \text { (D.11) }
\end{aligned}
$$

Substituting these into the right-hand side of (D.9), we have

$$
\begin{aligned}
& {\left[v_{\mathrm{tot}, x}^{(0)}(\boldsymbol{\phi}),\left[H_{0}^{(N)}(\boldsymbol{\phi}), v_{\mathrm{tot}, x}^{(0)}(\boldsymbol{\phi})\right]\right] } \\
= & \frac{\hbar^{2} e^{2} B^{2}}{m_{e}^{3}} N+\frac{\hbar^{2}}{m_{e}^{2}} \sum_{j=1}^{N}\left(\frac{\partial^{2} W}{\partial x^{2}}\right)\left(\mathbf{r}_{j}\right)+\frac{\hbar^{2} e^{2}}{m_{e}^{3}} \sum_{j=1}^{N}\left[B_{\mathrm{P}, z}\left(\mathbf{r}_{j}\right)\right]^{2}+\frac{2 \hbar^{2} e^{2} B}{m_{e}^{3}} \sum_{j=1}^{N} B_{\mathrm{P}, z}\left(\mathbf{r}_{j}\right) \\
+ & \frac{\hbar^{2} e}{2 m_{e}^{2}} \sum_{j=1}^{N}\left[v_{y, j}^{(0)}(\boldsymbol{\phi})\left(\frac{\partial B_{\mathrm{P}, z}}{\partial x}\right)\left(\mathbf{r}_{j}\right)+\left(\frac{\partial B_{\mathrm{P}, z}}{\partial x}\right)\left(\mathbf{r}_{j}\right) v_{y, j}^{(0)}(\boldsymbol{\phi})\right]
\end{aligned}
$$

Similarly we have

$$
\begin{aligned}
& {\left[v_{\mathrm{tot}, y}^{(0)}(\boldsymbol{\phi}),\left[H_{0}^{(N)}(\boldsymbol{\phi}), v_{\mathrm{tot}, y}^{(0)}(\boldsymbol{\phi})\right]\right] } \\
= & \frac{\hbar^{2} e^{2} B^{2}}{m_{e}^{3}} N+\frac{\hbar^{2}}{m_{e}^{2}} \sum_{j=1}^{N}\left(\frac{\partial^{2} W}{\partial y^{2}}\right)\left(\mathbf{r}_{j}\right)+\frac{\hbar^{2} e^{2}}{m_{e}^{3}} \sum_{j=1}^{N}\left[B_{\mathrm{P}, z}\left(\mathbf{r}_{j}\right)\right]^{2}+\frac{2 \hbar^{2} e^{2} B}{m_{e}^{3}} \sum_{j=1}^{N} B_{\mathrm{P}, z}\left(\mathbf{r}_{j}\right) \\
- & \frac{\hbar^{2} e}{2 m_{e}^{2}} \sum_{j=1}^{N}\left[v_{x, j}^{(0)}(\boldsymbol{\phi})\left(\frac{\partial B_{\mathrm{P}, z}}{\partial y}\right)\left(\mathbf{r}_{j}\right)+\left(\frac{\partial B_{\mathrm{P}, z}}{\partial y}\right)\left(\mathbf{r}_{j}\right) v_{x, j}^{(0)}(\boldsymbol{\phi})\right]
\end{aligned}
$$

by using the commutation relations (D.5) and (D.10).

From the expressions (D.12) and (D.13) for the double commutators, it is sufficient to evaluate the ground state expectation values of the last sums in both the right-hand sides. Since all the terms in the summands can be treated in the same way, we consider only the second term in the summand of the last sum in (D.13). Using the Schwarz inequality, we have

$$
\begin{aligned}
& \left|\sum_{j=1}^{N}\left\langle\Phi_{0, \mu}^{(N)}(\boldsymbol{\phi}),\left(\frac{\partial B_{\mathrm{P}, z}}{\partial y}\right)\left(\mathbf{r}_{j}\right) v_{x, j}^{(0)}(\boldsymbol{\phi}) \Phi_{0, \mu}^{(N)}(\boldsymbol{\phi})\right\rangle\right| \\
\leq & {\left[\sum_{i=1}^{N}\left\langle\Phi_{0, \mu}^{(N)}(\boldsymbol{\phi}),\left|\left(\frac{\partial B_{\mathrm{P}, z}}{\partial y}\right)\left(\mathbf{r}_{i}\right)\right|^{2} \Phi_{0, \mu}^{(N)}(\boldsymbol{\phi})\right\rangle\right]^{1 / 2}\left[\sum_{j=1}^{N}\left\langle\Phi_{0, \mu}^{(N)}(\boldsymbol{\phi}),\left[v_{x, j}^{(0)}(\boldsymbol{\phi})\right]^{2} \Phi_{0, \mu}^{(N)}(\boldsymbol{\phi})\right\rangle\right]^{1 / 2} }
\end{aligned}
$$




$$
\begin{aligned}
& \leq\left(\frac{2}{m_{e}}\right)^{1 / 2} N^{1 / 2}\left\|\frac{\partial B_{\mathrm{P}, z}}{\partial y}\right\|_{\infty}\left[\left\langle\Phi_{0, \mu}^{(N)}(\boldsymbol{\phi}), H_{0}^{(N)}(\boldsymbol{\phi}) \Phi_{0, \mu}^{(N)}(\boldsymbol{\phi})\right\rangle+N\|W\|_{\infty}\right]^{1 / 2} \\
& =\left(\frac{2}{m_{e}}\right)^{1 / 2} N^{1 / 2}\left\|\frac{\partial B_{\mathrm{P}, z}}{\partial y}\right\|_{\infty}\left[E_{0, \mu}^{(N)}(\boldsymbol{\phi})+N\|W\|_{\infty}\right]^{1 / 2} .
\end{aligned}
$$

For getting the second inequality, we have used the inequality,

$$
\sum_{j=1}^{N} \frac{m_{e}}{2}\left[v_{x, j}^{(0)}(\boldsymbol{\phi})\right]^{2} \leq H_{0}^{(N)}(\boldsymbol{\phi})+N\|W\|_{\infty}
$$

which follows from the assumption $W^{(2)} \geq 0$. By relying on the decay assumption (7.8) for the interaction $W^{(2)}$, we can prove [31] that the energy eigenvalue $E_{0, \mu}^{(N)}(\phi)$ is of order of $N$. See Appendix F for the detail. Consequently the right-hand side of the last line in (D.14) is of order of $N$.

\section{E Proofs of Theorems 7.6, 7.8 and 7.12}

We begin with rewriting the Hall conductance $\sigma_{x y}(\phi)$ of (D.1). Using the commutation relation (D.5), the summand in (D.1) can be written as

$$
\begin{aligned}
& \left\langle\Phi_{0, \mu}^{(N)}(\boldsymbol{\phi}), v_{\mathrm{tot}, y}^{(0)}(\boldsymbol{\phi}) \frac{1-Q\left(E_{0}^{(N)}(\boldsymbol{\phi})\right)}{\left[E_{0, \mu}^{(N)}(\boldsymbol{\phi})-H_{0}^{(N)}(\boldsymbol{\phi})\right]^{2}} v_{\mathrm{tot}, x}^{(0)}(\boldsymbol{\phi}) \Phi_{0, \mu}^{(N)}(\boldsymbol{\phi})\right\rangle-\text { c.c. } \\
= & -\frac{2 i m_{e}}{\hbar e B}\left\langle\Phi_{0, \mu}^{(N)}(\boldsymbol{\phi}), v_{\mathrm{tot}, y}^{(0)}(\boldsymbol{\phi}) \frac{1-Q\left(E_{0}^{(N)}(\boldsymbol{\phi})\right)}{E_{0, \mu}^{(N)}(\boldsymbol{\phi})-H_{0}^{(N)}(\boldsymbol{\phi})} v_{\mathrm{tot}, y}^{(0)}(\boldsymbol{\phi}) \Phi_{0, \mu}^{(N)}(\boldsymbol{\phi})\right\rangle \\
+ & {\left[\left\langle\Phi_{0, \mu}^{(N)}(\boldsymbol{\phi}), v_{\mathrm{tot}, y}^{(0)}(\boldsymbol{\phi}) \frac{1-Q\left(E_{0}^{(N)}(\boldsymbol{\phi})\right)}{\left[E_{0, \mu}^{(N)}(\boldsymbol{\phi})-H_{0}^{(N)}(\boldsymbol{\phi})\right]^{2}} I_{x}(\boldsymbol{\phi}) \Phi_{0, \mu}^{(N)}(\boldsymbol{\phi})\right\rangle-\text { c.c. }\right] }
\end{aligned}
$$

in the same way as in (4.21), where c.c. stands for the complex conjugate.

First we prove Theorem 7.6 by using the above expression (E.1). We assume $\mathbf{A}_{\mathrm{P}}=0$, i.e., $B_{\mathrm{P}, z}=0$. We treat only the case that the electrostatic potential $W$ is a function of the single variable $x$ only because we can treat the other case that $W$ is a function of the single variable $y$ only in the same way. From the assumptions and the expression (D.6) of $I_{x}(\phi)$, one has $I_{x}(\phi)=0$. Further we have

$$
\begin{aligned}
& 2 \sum_{\mu=1}^{q}\left\langle\Phi_{0, \mu}^{(N)}(\phi), v_{\text {tot }, y}^{(0)}(\phi) \frac{1-Q\left(E_{0}^{(N)}(\phi)\right)}{E_{0, \mu}^{(N)}(\phi)-H_{0}^{(N)}(\phi)} v_{\text {tot }, y}^{(0)}(\phi) \Phi_{0, \mu}^{(N)}(\phi)\right\rangle \\
= & \frac{\partial}{\partial \phi_{y}} \operatorname{Tr} v_{\text {tot }, y}^{(0)}(\phi) Q\left(E_{0}^{(N)}(\phi)\right)-\frac{N q}{m_{e}}
\end{aligned}
$$

in the same way as in Sec. 5. Combining these observations, (D.1) and (E.1), we obtain the desired result,

$$
\overline{\sigma_{x y}(\phi)}=-\frac{e^{2}}{h} \frac{N}{M}=-\frac{e^{2}}{h} \nu
$$


Now let us return to the general setting. We rewrite the right-hand side of (E.1) further. In the same way, we have

$$
\begin{aligned}
& \left\langle\Phi_{0, \mu}^{(N)}(\phi), v_{\mathrm{tot}, y}^{(0)}(\phi) \frac{1-Q\left(E_{0}^{(N)}(\phi)\right)}{\left[E_{0, \mu}^{(N)}(\phi)-H_{0}^{(N)}(\phi)\right]^{2}} I_{x}(\phi) \Phi_{0, \mu}^{(N)}(\phi)\right\rangle-\text { c.c. } \\
= & -\frac{i m_{e}}{\hbar e B}\left[\left\langle\Phi_{0, \mu}^{(N)}(\phi), v_{\mathrm{tot}, x}^{(0)}(\phi) \frac{1-Q\left(E_{0}^{(N)}(\phi)\right)}{E_{0, \mu}^{(N)}(\phi)-H_{0}^{(N)}(\phi)} I_{x}(\phi) \Phi_{0, \mu}^{(N)}(\phi)\right\rangle+\text { c.c. }\right] \\
+ & {\left[\left\langle\Phi_{0, \mu}^{(N)}(\phi), I_{x}(\phi) \frac{1-Q\left(E_{0}^{(N)}(\phi)\right)}{\left[E_{0, \mu}^{(N)}(\phi)-H_{0}^{(N)}(\phi)\right]^{2}} I_{y}(\phi) \Phi_{0, \mu}^{(N)}(\phi)\right\rangle-\text { c.c. }\right], }
\end{aligned}
$$

and

$$
\begin{aligned}
& \sum_{\mu=1}^{q}\left\langle\Phi_{0, \mu}^{(N)}(\phi), v_{\mathrm{tot}, x}^{(0)}(\phi) \frac{1-Q\left(E_{0}^{(N)}(\phi)\right)}{E_{0, \mu}^{(N)}(\phi)-H_{0}^{(N)}(\phi)} I_{x}(\phi) \Phi_{0, \mu}^{(N)}(\phi)\right\rangle+\text { c.c. } \\
= & \frac{\partial}{\partial \phi_{x}} \operatorname{Tr} I_{x}(\phi) Q\left(E_{0}^{(N)}(\phi)\right)+\frac{1}{m_{e} B} \sum_{j=1}^{N} \operatorname{Tr} B_{\mathrm{P}, z}\left(\mathbf{r}_{j}\right) Q\left(E_{0}^{(N)}(\phi)\right) .
\end{aligned}
$$

By combining (D.1), (E.1), (E.2), (E.4) and (E.5), the averaged Hall conductance can be written as

$$
\overline{\sigma_{x y}(\boldsymbol{\phi})}=-\frac{e^{2}}{h} \frac{N}{M}+\frac{e^{2}}{h} \frac{1}{M B q} \sum_{j=1}^{N} \overline{\operatorname{Tr} B_{\mathrm{P}, z}\left(\mathbf{r}_{j}\right) Q\left(E_{0}^{(N)}(\boldsymbol{\phi})\right)}+\overline{\Delta \sigma_{x y}(\boldsymbol{\phi})},
$$

where

$$
\Delta \sigma_{x y}(\boldsymbol{\phi})=\frac{i \hbar e^{2}}{L_{x} L_{y}} \frac{1}{q} \sum_{\mu=1}^{q}\left[\left\langle\Phi_{0, \mu}^{(N)}(\boldsymbol{\phi}), I_{x}(\boldsymbol{\phi}) \frac{1-Q\left(E_{0}^{(N)}(\boldsymbol{\phi})\right)}{\left[E_{0, \mu}^{(N)}(\boldsymbol{\phi})-H_{0}^{(N)}(\boldsymbol{\phi})\right]^{2}} I_{y}(\boldsymbol{\phi}) \Phi_{0, \mu}^{(N)}(\boldsymbol{\phi})\right\rangle-\text { c.c. }\right] \text {. }
$$

The sum in the right-hand side of (E.6) is easily evaluated as

$$
\left|\frac{e^{2}}{h} \frac{1}{M B q} \sum_{j=1}^{N} \overline{\operatorname{Tr} B_{\mathrm{P}, z}\left(\mathbf{r}_{j}\right) Q\left(E_{0}^{(N)}(\phi)\right)}\right| \leq \frac{e^{2}}{h} \frac{N}{M} \frac{\left\|B_{\mathrm{P}, z}\right\|_{\infty}}{B} .
$$

Next we estimate the right-hand side of (E.7). In the same way as in the preceding Appendix D, we have

$$
\left|\Delta \sigma_{x y}(\phi)\right| \leq\left(\frac{\hbar \omega_{c}}{\Delta E}\right)^{3} \frac{e^{2}}{h} \frac{N}{M} \delta^{\prime}(\phi)
$$

with

$$
\delta^{\prime}(\boldsymbol{\phi})=\frac{m_{e}}{\left(\hbar \omega_{c}\right)^{2} N} \prod_{s=x, y} \sqrt{\frac{1}{q} \sum_{\mu=1}^{q}\left\langle\Phi_{0, \mu}^{(N)}(\boldsymbol{\phi}),\left[I_{s}(\boldsymbol{\phi}),\left[H_{0}^{(N)}(\boldsymbol{\phi}), I_{s}(\boldsymbol{\phi})\right]\right] \Phi_{0, \mu}^{(N)}(\boldsymbol{\phi})\right\rangle} .
$$

In the rest of this Appendix, we will show

$$
\delta^{\prime}(\phi) \leq \tilde{\delta}^{\prime},
$$


where $\tilde{\delta}^{\prime}$ is independent of $\boldsymbol{\phi}$, the number $N$ of the electrons and of the sizes $L_{x}, L_{y}$ of the system. But $\tilde{\delta}^{\prime}$ is a continuous function of the norms $\left\|D^{(m, n)} B_{\mathrm{P}, z}\right\|_{\infty},\left\|D^{(m, n)} W\right\|_{\infty}$ and satisfies $\tilde{\delta}^{\prime}=0$ in the special point with $\mathbf{A}_{\mathrm{P}}=0$ and $W=0$. Therefore $\tilde{\delta}^{\prime}$ becomes small for the weak potentials $\mathbf{A}_{\mathrm{P}}$ and $W$. Combining (E.6), (E.8), (E.9) and (E.11), we have the desired result,

$$
-\frac{e^{2}}{h} \nu(1+\delta) \leq \overline{\sigma_{x y}(\phi)} \leq-\frac{e^{2}}{h} \nu(1-\delta),
$$

with

$$
\delta=\frac{\left\|B_{\mathrm{P}, z}\right\|_{\infty}}{B}+\left(\frac{\hbar \omega_{c}}{\Delta E}\right)^{3} \tilde{\delta}^{\prime} .
$$

In passing, we remark that we can obtain a similar bound for the non-averaged Hall conductance $\sigma_{x y}(\phi)$ to (E.12) by using (D.4) and (D.10) for the first term in the righthand side of (E.1).

In order to prove the bound (E.11), it is sufficient to estimate the ground state expectation values of the double commutators $\left[I_{s}(\phi),\left[H_{0}^{(N)}(\phi), I_{s}(\phi)\right]\right]$ in (E.10). To this end, let us calculate the double commutators. In the following, we will consider only the case with $s=x$ because we can treat the case with $s=y$ exactly in the same way. Note that

$$
\begin{aligned}
{\left[H_{0}^{(N)}(\boldsymbol{\phi}), I_{x}(\boldsymbol{\phi})\right] } & =\frac{m_{e}}{2 e B} \sum_{j=1}^{N}\left[\left(v_{x, j}^{(0)}(\boldsymbol{\phi})\right)^{2}+\left(v_{y, j}^{(0)}(\boldsymbol{\phi})\right)^{2},\left(\frac{\partial W}{\partial y}\right)\left(\mathbf{r}_{j}\right)\right] \\
& -\frac{m_{e}}{4 B} \sum_{j=1}^{N}\left[\left(v_{x, j}^{(0)}(\boldsymbol{\phi})\right)^{2}+\left(v_{y, j}^{(0)}(\boldsymbol{\phi})\right)^{2}, B_{\mathrm{P}, z}\left(\mathbf{r}_{j}\right) v_{x, j}^{(0)}(\boldsymbol{\phi})+v_{x, j}^{(0)}(\boldsymbol{\phi}) B_{\mathrm{P}, z}\left(\mathbf{r}_{j}\right)\right] \\
& -\frac{1}{2 B} \sum_{j=1}^{N}\left[W\left(\mathbf{r}_{j}\right), B_{\mathrm{P}, z}\left(\mathbf{r}_{j}\right) v_{x, j}^{(0)}(\boldsymbol{\phi})+v_{x, j}^{(0)}(\boldsymbol{\phi}) B_{\mathrm{P}, z}\left(\mathbf{r}_{j}\right)\right] \\
& -\frac{1}{2 B} \sum_{i, j} \sum_{\ell=1}^{N}\left[W^{(2)}\left(\mathbf{r}_{i}-\mathbf{r}_{j}\right), B_{\mathrm{P}, z}\left(\mathbf{r}_{\ell}\right) v_{x, \ell}^{(0)}(\boldsymbol{\phi})+v_{x, \ell}^{(0)}(\boldsymbol{\phi}) B_{\mathrm{P}, z}\left(\mathbf{r}_{\ell}\right)\right] \\
& =\sum_{s=1}^{3} J_{x}^{(0, s)}+J_{x}^{(x)}(\boldsymbol{\phi})+\sum_{s=1}^{3} J_{x}^{(y, s)}(\boldsymbol{\phi})+J_{x}^{(x x)}(\boldsymbol{\phi})+J_{x}^{(x y)}(\boldsymbol{\phi}),
\end{aligned}
$$

where the operators in the last line are given by

$$
\begin{gathered}
J_{x}^{(0,1)}=-\frac{i \hbar}{m_{e} B} \sum_{j=1}^{N} B_{\mathrm{P}, z}\left(\mathbf{r}_{j}\right)\left(\frac{\partial W}{\partial x}\right)\left(\mathbf{r}_{j}\right), \\
J_{x}^{(0,2)}=-\frac{i \hbar^{3}}{4 m_{e}^{2} B} \sum_{j=1}^{N}\left[\left(\frac{\partial^{3} B_{\mathrm{P}, z}}{\partial x^{3}}\right)\left(\mathbf{r}_{j}\right)+\left(\frac{\partial^{3} B_{\mathrm{P}, z}}{\partial x \partial y^{2}}\right)\left(\mathbf{r}_{j}\right)\right], \\
J_{x}^{(0,3)}=-\frac{i \hbar}{m_{e} B} \sum_{i, j}\left[B_{\mathrm{P}, z}\left(\mathbf{r}_{i}\right)-B_{\mathrm{P}, z}\left(\mathbf{r}_{j}\right)\right]\left(\frac{\partial W^{(2)}}{\partial x}\right)\left(\mathbf{r}_{i}-\mathbf{r}_{j}\right), \\
J_{x}^{(x)}(\boldsymbol{\phi})=-\frac{i \hbar}{2 e B} \sum_{j=1}^{N}\left[v_{x, j}^{(0)}(\boldsymbol{\phi})\left(\frac{\partial^{2} W}{\partial x \partial y}\right)\left(\mathbf{r}_{j}\right)+\left(\frac{\partial^{2} W}{\partial x \partial y}\right)\left(\mathbf{r}_{j}\right) v_{x, j}^{(0)}(\boldsymbol{\phi})\right],
\end{gathered}
$$


Revisiting the Charge Transport in Quantum Hall Systems

$$
\begin{gathered}
J_{x}^{(y, 1)}(\boldsymbol{\phi})=-\frac{i \hbar}{2 e B} \sum_{j=1}^{N}\left[v_{y, j}^{(0)}(\boldsymbol{\phi})\left(\frac{\partial^{2} W}{\partial y^{2}}\right)\left(\mathbf{r}_{j}\right)+\left(\frac{\partial^{2} W}{\partial y^{2}}\right)\left(\mathbf{r}_{j}\right) v_{y, j}^{(0)}(\boldsymbol{\phi})\right] \\
J_{x}^{(y, 2)}(\boldsymbol{\phi})=-\frac{i \hbar e}{2 m_{e}} \sum_{j=1}^{N}\left[v_{y, j}^{(0)}(\boldsymbol{\phi}) B_{\mathrm{P}, z}\left(\mathbf{r}_{j}\right)+B_{\mathrm{P}, z}\left(\mathbf{r}_{j}\right) v_{y, j}^{(0)}(\boldsymbol{\phi})\right] \\
J_{x}^{(y, 3)}(\boldsymbol{\phi})=-\frac{i \hbar e}{2 m_{e} B} \sum_{j=1}^{N}\left\{v_{y, j}^{(0)}(\boldsymbol{\phi})\left[B_{\mathrm{P}, z}\left(\mathbf{r}_{j}\right)\right]^{2}+\left[B_{\mathrm{P}, z}\left(\mathbf{r}_{j}\right)\right]^{2} v_{y, j}^{(0)}(\boldsymbol{\phi})\right\} \\
J_{x}^{(x x)}(\boldsymbol{\phi})=\frac{i \hbar}{B} \sum_{j=1}^{N} v_{x, j}^{(0)}(\boldsymbol{\phi})\left(\frac{\partial B_{\mathrm{P}, z}}{\partial x}\right)\left(\mathbf{r}_{j}\right) v_{x, j}^{(0)}(\boldsymbol{\phi})
\end{gathered}
$$

and

$$
J_{x}^{(x y)}(\boldsymbol{\phi})=\frac{i \hbar}{2 B} \sum_{j=1}^{N}\left[v_{x, j}^{(0)}(\boldsymbol{\phi})\left(\frac{\partial B_{\mathrm{P}, z}}{\partial y}\right)\left(\mathbf{r}_{j}\right) v_{y, j}^{(0)}(\boldsymbol{\phi})+v_{y, j}^{(0)}(\boldsymbol{\phi})\left(\frac{\partial B_{\mathrm{P}, z}}{\partial y}\right)\left(\mathbf{r}_{j}\right) v_{x, j}^{(0)}(\boldsymbol{\phi})\right] .
$$

Hence the double commutator becomes

$$
\begin{aligned}
{\left[I_{x}(\boldsymbol{\phi}),\left[H_{0}^{(N)}(\boldsymbol{\phi}), I_{x}(\boldsymbol{\phi})\right]\right] } & =\sum_{s=1}^{3}\left[I_{x}(\boldsymbol{\phi}), J_{x}^{(0, s)}\right]+\left[I_{x}(\boldsymbol{\phi}), J_{x}^{(x)}(\boldsymbol{\phi})\right]+\sum_{s=1}^{3}\left[I_{x}(\boldsymbol{\phi}), J_{x}^{(y, s)}(\boldsymbol{\phi})\right] \\
& +\left[I_{x}(\boldsymbol{\phi}), J_{x}^{(x x)}(\boldsymbol{\phi})\right]+\left[I_{x}(\boldsymbol{\phi}), J_{x}^{(x y)}\right] .
\end{aligned}
$$

In order to prove the boundedness of $\delta^{\prime}(\phi)$ of (E.10), we shall show that the ground state expectation values for all the commutators in this right-hand side are of order $N$.

The three commutators in the first sum in the right-hand side of (E.24) are calculated as

$$
\begin{gathered}
\\
{\left[I_{x}(\boldsymbol{\phi}), J_{x}^{(0,1)}\right]} \\
=\frac{\hbar^{2}}{m_{e}^{2} B^{2}} \sum_{j=1}^{N}\left\{\left[B_{\mathrm{P}, z}\left(\mathbf{r}_{j}\right)\right]^{2}\left(\frac{\partial^{2} W}{\partial x^{2}}\right)\left(\mathbf{r}_{j}\right)+B_{\mathrm{P}, z}\left(\mathbf{r}_{j}\right)\left(\frac{\partial B_{\mathrm{P}, z}}{\partial x}\right)\left(\mathbf{r}_{j}\right)\left(\frac{\partial W}{\partial x}\right)\left(\mathbf{r}_{j}\right)\right\} \\
{\left[I_{x}(\boldsymbol{\phi}), J_{x}^{(0,2)}\right]=\frac{\hbar^{4}}{4 m_{e}^{3} B^{2}} \sum_{j=1}^{N} B_{\mathrm{P}, z}\left(\mathbf{r}_{j}\right)\left[\left(\frac{\partial^{4} B_{\mathrm{P}, z}}{\partial x^{4}}\right)\left(\mathbf{r}_{j}\right)+\left(\frac{\partial^{4} B_{\mathrm{P}, z}}{\partial x^{2} \partial y^{2}}\right)\left(\mathbf{r}_{j}\right)\right]}
\end{gathered}
$$

and

$$
\begin{aligned}
& {\left[I_{x}(\boldsymbol{\phi}), J_{x}^{(0,3)}\right] } \\
= & \frac{\hbar^{2}}{m_{e}^{2} B^{2}} \sum_{i, j}\left[B_{\mathrm{P}, z}\left(\mathbf{r}_{i}\right)-B_{\mathrm{P}, z}\left(\mathbf{r}_{j}\right)\right]^{2}\left(\frac{\partial^{2} W^{(2)}}{\partial x^{2}}\right)\left(\mathbf{r}_{i}-\mathbf{r}_{j}\right) \\
+ & \frac{\hbar^{2}}{m_{e}^{2} B^{2}} \sum_{i, j}\left[B_{\mathrm{P}, z}\left(\mathbf{r}_{i}\right)\left(\frac{\partial B_{\mathrm{P}, z}}{\partial x}\right)\left(\mathbf{r}_{i}\right)-B_{\mathrm{P}, z}\left(\mathbf{r}_{j}\right)\left(\frac{\partial B_{\mathrm{P}, z}}{\partial x}\right)\left(\mathbf{r}_{j}\right)\right]\left(\frac{\partial W^{(2)}}{\partial x}\right)\left(\mathbf{r}_{i}-\mathbf{r}_{j}\right) .
\end{aligned}
$$


Clearly the first two ones are of order $N$ from the assumptions. The ground state expectation value of the rest one is evaluated as

$$
\begin{aligned}
& \left|\left\langle\Phi_{0, \mu}^{(N)}(\boldsymbol{\phi}),\left[I_{x}(\boldsymbol{\phi}), J_{x}^{(0,3)}\right] \Phi_{0, \mu}^{(N)}(\boldsymbol{\phi})\right\rangle\right| \\
\leq & \frac{4 \hbar^{2}}{m_{e}^{2}}\left(\frac{\left\|B_{\mathrm{P}, z}\right\|_{\infty}}{B}\right)^{2} \sum_{i, j}\left\langle\Phi_{0, \mu}^{(N)}(\boldsymbol{\phi}),\left|\left(\frac{\partial^{2} W^{(2)}}{\partial x^{2}}\right)\left(\mathbf{r}_{i}-\mathbf{r}_{j}\right)\right| \Phi_{0, \mu}^{(N)}(\boldsymbol{\phi})\right\rangle \\
+ & \frac{2 \hbar^{2}}{m_{e}^{2} B} \frac{\left\|B_{\mathrm{P}, z}\right\|_{\infty}}{B}\left\|\frac{\partial B_{\mathrm{P}, z}}{\partial x}\right\|_{\infty} \sum_{i, j}\left\langle\Phi_{0, \mu}^{(N)}(\boldsymbol{\phi}),\left|\left(\frac{\partial W^{(2)}}{\partial x}\right)\left(\mathbf{r}_{i}-\mathbf{r}_{j}\right)\right| \Phi_{0, \mu}^{(N)}(\boldsymbol{\phi})\right\rangle \\
\leq & \frac{4 \alpha_{\mathrm{int}} \hbar \omega_{c}}{m_{e}} \frac{\left\|B_{\mathrm{P}, z}\right\|_{\infty}}{B}\left(\frac{\left\|B_{\mathrm{P}, z}\right\|_{\infty}}{B}+\frac{\ell_{B}}{2 B}\left\|\frac{\partial B_{\mathrm{P}, z}}{\partial x}\right\|_{\infty}\right) \\
& \times \sum_{i, j}\left\langle\Phi_{0, \mu}^{(N)}(\boldsymbol{\phi}), W^{(2)}\left(\mathbf{r}_{i}-\mathbf{r}_{j}\right) \Phi_{0, \mu}^{(N)}(\boldsymbol{\phi})\right\rangle,
\end{aligned}
$$

where we have used the assumptions (7.18) and (7.19). Further, by using the inequality

$$
\begin{aligned}
\sum_{i, j}\left\langle\Phi_{0, \mu}^{(N)}(\boldsymbol{\phi}), W^{(2)}\left(\mathbf{r}_{i}-\mathbf{r}_{j}\right) \Phi_{0, \mu}^{(N)}(\boldsymbol{\phi})\right\rangle & \leq\left\langle\Phi_{0, \mu}^{(N)}(\boldsymbol{\phi}), H_{0}^{(N)}(\boldsymbol{\phi}) \Phi_{0, \mu}^{(N)}(\boldsymbol{\phi})\right\rangle+N\|W\| \\
& =E_{0, \mu}^{(N)}(\boldsymbol{\phi})+N\|W\|,
\end{aligned}
$$

we have

$$
\begin{aligned}
\left|\left\langle\Phi_{0, \mu}^{(N)}(\boldsymbol{\phi}),\left[I_{x}(\boldsymbol{\phi}), J_{x}^{(0,3)}\right] \Phi_{0, \mu}^{(N)}(\boldsymbol{\phi})\right\rangle\right| & \leq \frac{4 \alpha_{\mathrm{int}} \hbar \omega_{c}}{m_{e}} \frac{\left\|B_{\mathrm{P}, z}\right\|_{\infty}}{B}\left(\frac{\left\|B_{\mathrm{P}, z}\right\|_{\infty}}{B}+\frac{\ell_{B}}{2 B}\left\|\frac{\partial B_{\mathrm{P}, z}}{\partial x}\right\|_{\infty}\right) \\
& \times\left(E_{0, \mu}^{(N)}(\boldsymbol{\phi})+N\|W\|\right) .
\end{aligned}
$$

Since the ground state energy $E_{0, \mu}^{(N)}(\phi)$ is of order $N$ as shown in Appendix F, this righthand side is of order $N$. We remark that, when $\mathbf{A}_{\mathrm{P}}=0$, we do not need the assumptions (7.18) and (7.19) because all the operators $J_{x}^{(0, s)}$ are vanishing.

Note that

$$
\begin{aligned}
{\left[I_{x}(\boldsymbol{\phi}), J_{x}^{(x)}(\boldsymbol{\phi})\right] } & =\frac{\hbar^{2}}{m_{e} e^{2} B^{2}} \sum_{j=1}^{N}\left[\left(\frac{\partial^{2} W}{\partial x \partial y}\right)\left(\mathbf{r}_{j}\right)\right]^{2} \\
& +\frac{\hbar^{2}}{2 m_{e} e B^{2}} \sum_{j=1}^{N}\left\{v_{x, j}^{(0)}(\boldsymbol{\phi}), B_{\mathrm{P}, z}\left(\mathbf{r}_{j}\right)\left(\frac{\partial^{3} W}{\partial x^{2} \partial y}\right)\left(\mathbf{r}_{j}\right)\right\} \\
& -\frac{\hbar^{2}}{2 m_{e} e B^{2}} \sum_{j=1}^{N}\left\{v_{x, j}^{(0)}(\boldsymbol{\phi}),\left(\frac{\partial B_{\mathrm{P}, z}}{\partial x}\right)\left(\mathbf{r}_{j}\right)\left(\frac{\partial^{2} W}{\partial x \partial y}\right)\left(\mathbf{r}_{j}\right)\right\}, \\
{\left[I_{x}(\boldsymbol{\phi}), J_{x}^{(y, 1)}(\boldsymbol{\phi})\right]=} & \frac{\hbar^{2}}{m_{e} e^{2} B^{2}} \sum_{j=1}^{N}\left[\left(\frac{\partial^{2} W}{\partial y^{2}}\right)\left(\mathbf{r}_{j}\right)\right]^{2} \\
+ & \frac{\hbar^{2}}{2 m_{e} e B^{2}} \sum_{j=1}^{N}\left\{v_{y, j}^{(0)}(\boldsymbol{\phi}), B_{\mathrm{P}, z}\left(\mathbf{r}_{j}\right)\left(\frac{\partial^{3} W}{\partial x \partial y y^{2}}\right)\left(\mathbf{r}_{j}\right)\right\}
\end{aligned}
$$


Revisiting the Charge Transport in Quantum Hall Systems

$$
\begin{aligned}
- & \frac{\hbar^{2}}{2 m_{e} e B^{2}} \sum_{j=1}^{N}\left\{v_{x, j}^{(0)}(\boldsymbol{\phi}),\left(\frac{\partial B_{\mathrm{P}, z}}{\partial y}\right)\left(\mathbf{r}_{j}\right)\left(\frac{\partial^{2} W}{\partial y^{2}}\right)\left(\mathbf{r}_{j}\right)\right\} \\
+ & \frac{\hbar^{2}}{m_{e}^{2} B} \sum_{j=1}^{N} B_{\mathrm{P}, z}\left(\mathbf{r}_{j}\right)\left(\frac{\partial^{2} W}{\partial y^{2}}\right)\left(\mathbf{r}_{j}\right)+\frac{\hbar^{2}}{m_{e}^{2} B^{2}} \sum_{j=1}^{N}\left[B_{\mathrm{P}, z}\left(\mathbf{r}_{j}\right)\right]^{2}\left(\frac{\partial^{2} W}{\partial y^{2}}\right)\left(\mathbf{r}_{j}\right), \\
{\left[I_{x}(\boldsymbol{\phi}), J_{x}^{(y, 2)}(\boldsymbol{\phi})\right] } & =\frac{\hbar^{2}}{m_{e}^{2} B} \sum_{j=1}^{N} B_{\mathrm{P}, z}\left(\mathbf{r}_{j}\right)\left(\frac{\partial^{2} W}{\partial y^{2}}\right)\left(\mathbf{r}_{j}\right) \\
& +\frac{\hbar^{2} e}{2 m_{e}^{2} B} \sum_{j=1}^{N}\left\{v_{y, j}^{(0)}(\boldsymbol{\phi}), B_{\mathrm{P}, z}\left(\mathbf{r}_{j}\right)\left(\frac{\partial B_{\mathrm{P}, z}}{\partial x}\right)\left(\mathbf{r}_{j}\right)\right\} \\
& -\frac{\hbar^{2} e}{2 m_{e}^{2} B} \sum_{j=1}^{N}\left\{v_{x, j}^{(0)}(\boldsymbol{\phi}), B_{\mathrm{P}, z}\left(\mathbf{r}_{j}\right)\left(\frac{\partial B_{\mathrm{P}, z}}{\partial y}\right)\left(\mathbf{r}_{j}\right)\right\} \\
& +\frac{\hbar^{2} e^{2}}{m_{e}^{3}} \sum_{j=1}^{N}\left[B_{\mathrm{P}, z}\left(\mathbf{r}_{j}\right)\right]^{2}+\frac{\hbar^{2} e^{2}}{m_{e}^{3} B} \sum_{j=1}^{N}\left[B_{\mathrm{P}, z}\left(\mathbf{r}_{j}\right)\right]^{3},
\end{aligned}
$$

and

$$
\begin{aligned}
{\left[I_{x}(\boldsymbol{\phi}), J_{x}^{(y, 3)}(\boldsymbol{\phi})\right] } & =\frac{\hbar^{2}}{m_{e}^{2} B^{2}} \sum_{j=1}^{N}\left[B_{\mathrm{P}, z}\left(\mathbf{r}_{j}\right)\right]^{2}\left(\frac{\partial^{2} W}{\partial y^{2}}\right)\left(\mathbf{r}_{j}\right) \\
& +\frac{\hbar^{2} e}{m_{e}^{2} B^{2}} \sum_{j=1}^{N}\left\{v_{y, j}^{(0)}(\boldsymbol{\phi}),\left[B_{\mathrm{P}, z}\left(\mathbf{r}_{j}\right)\right]^{2}\left(\frac{\partial B_{\mathrm{P}, z}}{\partial x}\right)\left(\mathbf{r}_{j}\right)\right\} \\
& -\frac{\hbar^{2} e}{2 m_{e}^{2} B^{2}} \sum_{j=1}^{N}\left\{v_{x, j}^{(0)}(\boldsymbol{\phi}),\left[B_{\mathrm{P}, z}\left(\mathbf{r}_{j}\right)\right]^{2}\left(\frac{\partial B_{\mathrm{P}, z}}{\partial y}\right)\left(\mathbf{r}_{j}\right)\right\} \\
& +\frac{\hbar^{2} e^{2}}{m_{e}^{3} B} \sum_{j=1}^{N}\left[B_{\mathrm{P}, z}\left(\mathbf{r}_{j}\right)\right]^{3}+\frac{\hbar^{2} e^{2}}{m_{e}^{3} B^{2}} \sum_{j=1}^{N}\left[B_{\mathrm{P}, z}\left(\mathbf{r}_{j}\right)\right]^{4}
\end{aligned}
$$

where $\{X, Y\}=X Y+Y X$ for operators $X, Y$. Since the terms including the velocity operators $v_{s, j}^{(0)}(\phi)$ in these right-hand sides can be estimated in the same way as in the previous Appendix D, we can get the desired estimates of order $N$ for their ground state expectation values.

Note that

$$
\begin{aligned}
& {\left[I_{x}(\boldsymbol{\phi}), J_{x}^{(x x)}(\boldsymbol{\phi})\right] } \\
= & -\frac{\hbar^{2}}{m_{e} e B^{2}} \sum_{j=1}^{N}\left\{v_{x, j}^{(0)}(\boldsymbol{\phi}),\left(\frac{\partial B_{\mathrm{P}, z}}{\partial x}\right)\left(\mathbf{r}_{j}\right)\left(\frac{\partial^{2} W}{\partial x \partial y}\right)\left(\mathbf{r}_{j}\right)\right\} \\
+ & \frac{\hbar^{2}}{m_{e} B^{2}} \sum_{j=1}^{N} v_{x, j}^{(0)}(\boldsymbol{\phi})\left[2\left|\left(\frac{\partial B_{\mathrm{P}, z}}{\partial x}\right)\left(\mathbf{r}_{j}\right)\right|^{2}-B_{\mathrm{P}, z}\left(\mathbf{r}_{j}\right)\left(\frac{\partial^{2} B_{\mathrm{P}, z}}{\partial x^{2}}\right)\left(\mathbf{r}_{j}\right)\right] v_{x, j}^{(0)}(\boldsymbol{\phi}) \\
- & \frac{\hbar^{4}}{2 m_{e}^{3} B^{2}} \sum_{j=1}^{N}\left[\left|\left(\frac{\partial^{2} B_{\mathrm{P}, z}}{\partial x^{2}}\right)\left(\mathbf{r}_{j}\right)\right|^{2}+\left(\frac{\partial B_{\mathrm{P}, z}}{\partial x}\right)\left(\mathbf{r}_{j}\right)\left(\frac{\partial^{3} B_{\mathrm{P}, z}}{\partial x^{3}}\right)\left(\mathbf{r}_{j}\right)\right] .
\end{aligned}
$$


Revisiting the Charge Transport in Quantum Hall Systems

Clearly the ground state expectation values for the first and third sums can be evaluated in the same way as in the above. For the second sum, we have

$$
\begin{aligned}
& \left|\sum_{j=1}^{N}\left\langle\Phi_{0, \mu}^{(N)}(\boldsymbol{\phi}), v_{x, j}^{(0)}(\boldsymbol{\phi})\left[2\left|\left(\frac{\partial B_{\mathrm{P}, z}}{\partial x}\right)\left(\mathbf{r}_{j}\right)\right|^{2}-B_{\mathrm{P}, z}\left(\mathbf{r}_{j}\right)\left(\frac{\partial^{2} B_{\mathrm{P}, z}}{\partial x^{2}}\right)\left(\mathbf{r}_{j}\right)\right] v_{x, j}^{(0)}(\boldsymbol{\phi}) \Phi_{0, \mu}^{(N)}(\boldsymbol{\phi})\right\rangle\right| \\
& \leq\left(2\left\|\frac{\partial B_{\mathrm{P}, z}}{\partial x}\right\|_{\infty}^{2}+\left\|B_{\mathrm{P}, z}\right\|_{\infty}\left\|\frac{\partial^{2} B_{\mathrm{P}, z}}{\partial x^{2}}\right\|_{\infty}\right) \sum_{j=1}^{N}\left\langle\Phi_{0, \mu}^{(N)}(\phi),\left(v_{x, j}^{(0)}(\boldsymbol{\phi})\right)^{2} \Phi_{0, \mu}^{(N)}(\boldsymbol{\phi})\right\rangle \\
& \leq \frac{2}{m_{e}}\left(2\left\|\frac{\partial B_{\mathrm{P}, z}}{\partial x}\right\|_{\infty}^{2}+\left\|B_{\mathrm{P}, z}\right\|_{\infty}\left\|\frac{\partial^{2} B_{\mathrm{P}, z}}{\partial x^{2}}\right\|_{\infty}\right)\left[\left\langle\Phi_{0, \mu}^{(N)}(\boldsymbol{\phi}), H_{0}^{(N)}(\boldsymbol{\phi}) \Phi_{0, \mu}^{(N)}(\boldsymbol{\phi})\right\rangle+N\|W\|_{\infty}\right] \\
& =\frac{2}{m_{e}}\left(2\left\|\frac{\partial B_{\mathrm{P}, z}}{\partial x}\right\|_{\infty}^{2}+\left\|B_{\mathrm{P}, z}\right\|_{\infty}\left\|\frac{\partial^{2} B_{\mathrm{P}, z}}{\partial x^{2}}\right\|_{\infty}\right)\left[E_{0, \mu}^{(N)}(\boldsymbol{\phi})+N\|W\|_{\infty}\right] .
\end{aligned}
$$

Thus the corresponding contribution is of order $N$.

Finally let us compute the ground state expectation value of $\left[I_{x}(\phi), J_{x}^{(x y)}(\phi)\right]$. In order to make this task easier, we decompose the operator $I_{x}(\phi)$ into two parts as

$$
I_{x}(\phi)=I_{x}^{(1)}+I_{x}^{(2)}(\phi)
$$

with

$$
I_{x}^{(1)}=\frac{1}{e B} \sum_{j=1}^{N}\left(\frac{\partial W}{\partial y}\right)\left(\mathbf{r}_{j}\right)
$$

and

$$
I_{x}^{(2)}(\boldsymbol{\phi})=-\frac{1}{2 B} \sum_{j=1}^{N}\left[B_{\mathrm{P}, z}\left(\mathbf{r}_{j}\right) v_{x, j}^{(0)}(\boldsymbol{\phi})+v_{x, j}^{(0)}(\boldsymbol{\phi}) B_{\mathrm{P}, z}\left(\mathbf{r}_{j}\right)\right]
$$

Note that

$$
\begin{aligned}
{\left[I_{x}^{(1)}, J_{x}^{(x y)}(\boldsymbol{\phi})\right] } & =-\frac{\hbar^{2}}{2 m_{e} e B^{2}} \sum_{j=1}^{N}\left\{v_{x, j}^{(0)}(\boldsymbol{\phi}),\left(\frac{\partial B_{\mathrm{P}, z}}{\partial y}\right)\left(\mathbf{r}_{j}\right)\left(\frac{\partial^{2} W}{\partial y^{2}}\right)\left(\mathbf{r}_{j}\right)\right\} \\
& -\frac{\hbar^{2}}{2 m_{e} e B^{2}} \sum_{j=1}^{N}\left\{v_{y, j}^{(0)}(\boldsymbol{\phi}),\left(\frac{\partial B_{\mathrm{P}, z}}{\partial y}\right)\left(\mathbf{r}_{j}\right)\left(\frac{\partial^{2} W}{\partial x \partial y}\right)\left(\mathbf{r}_{j}\right)\right\}
\end{aligned}
$$

and

$$
\left[I_{x}^{(2)}(\phi), J_{x}^{(x y)}(\phi)\right]=K_{x}^{(x x)}(\phi)+K_{x}^{(x y)}(\phi)+K_{x}^{(x)}(\phi)+K_{x}^{(0)}
$$

with

$$
\begin{gathered}
K_{x}^{(x x)}(\boldsymbol{\phi})=\frac{\hbar^{2}}{m_{e} B^{2}} \sum_{j=1}^{N} v_{x, j}^{(0)}(\boldsymbol{\phi})\left[\left(\frac{\partial B_{\mathrm{P}, z}}{\partial y}\right)\left(\mathbf{r}_{j}\right)\right]^{2} v_{x, j}^{(0)}(\boldsymbol{\phi}), \\
K_{x}^{(x y)}(\boldsymbol{\phi})=-\frac{\hbar^{2}}{2 m_{e} B^{2}} \sum_{j=1}^{N}\left[v_{x, j}^{(0)}(\boldsymbol{\phi}) B_{\mathrm{P}, z}\left(\mathbf{r}_{j}\right)\left(\frac{\partial^{2} B_{\mathrm{P}, z}}{\partial x \partial y}\right)\left(\mathbf{r}_{j}\right) v_{y, j}^{(0)}(\boldsymbol{\phi})+(x \leftrightarrow y)\right] \\
+\frac{\hbar^{2}}{2 m_{e} B^{2}} \sum_{j=1}^{N}\left[v_{x, j}^{(0)}(\boldsymbol{\phi})\left(\frac{\partial B_{\mathrm{P}, z}}{\partial x}\right)\left(\mathbf{r}_{j}\right)\left(\frac{\partial B_{\mathrm{P}, z}}{\partial y}\right)\left(\mathbf{r}_{j}\right) v_{y, j}^{(0)}(\boldsymbol{\phi})+(x \leftrightarrow y)\right],
\end{gathered}
$$




$$
\begin{aligned}
K_{x}^{(x)}(\boldsymbol{\phi}) & =-\frac{\hbar^{2} e}{2 m_{e}^{2} B} \sum_{j=1}^{N}\left\{v_{x, j}^{(0)}(\boldsymbol{\phi}), B_{\mathrm{P}, z}\left(\mathbf{r}_{j}\right)\left(\frac{\partial B_{\mathrm{P}, z}}{\partial y}\right)\left(\mathbf{r}_{j}\right)\right\} \\
& -\frac{\hbar^{2} e}{2 m_{e}^{2} B^{2}} \sum_{j=1}^{N}\left\{v_{x, j}^{(0)}(\boldsymbol{\phi}),\left[B_{\mathrm{P}, z}\left(\mathbf{r}_{j}\right)\right]^{2}\left(\frac{\partial B_{\mathrm{P}, z}}{\partial y}\right)\left(\mathbf{r}_{j}\right)\right\},
\end{aligned}
$$

and

$$
\begin{aligned}
K_{x}^{(0)} & =-\frac{\hbar^{4}}{4 m_{e}^{3} B^{2}} \sum_{j=1}^{N}\left[2\left(\frac{\partial B_{\mathrm{P}, z}}{\partial y}\right)\left(\mathbf{r}_{j}\right)\left(\frac{\partial^{3} B_{\mathrm{P}, z}}{\partial x^{2} \partial y}\right)\left(\mathbf{r}_{j}\right)+\left(\frac{\partial^{2} B_{\mathrm{P}, z}}{\partial x^{2}}\right)\left(\mathbf{r}_{j}\right)\left(\frac{\partial^{2} B_{\mathrm{P}, z}}{\partial y^{2}}\right)\left(\mathbf{r}_{j}\right)\right] \\
& -\frac{\hbar^{4}}{4 m_{e}^{3} B^{2}} \sum_{j=1}^{N}\left[\left(\frac{\partial^{2} B_{\mathrm{P}, z}}{\partial x \partial y}\right)\left(\mathbf{r}_{j}\right)\right]^{2} \cdot
\end{aligned}
$$

Hence all the contributions except for that for $K_{x}^{(x y)}(\phi)$ can be evaluated in the same way as in the above. We shall show that the ground state expectation value of $K_{x}^{(x y)}(\phi)$ is of order $N$, too. Using the Schwarz inequality, we have

$$
\begin{gathered}
\left|\sum_{j=1}^{N}\left\langle\Phi_{0, \mu}^{(N)}(\boldsymbol{\phi}), v_{x, j}^{(0)}(\boldsymbol{\phi}) B_{\mathrm{P}, z}\left(\mathbf{r}_{j}\right)\left(\frac{\partial^{2} B_{\mathrm{P}, z}}{\partial x \partial y}\right)\left(\mathbf{r}_{j}\right) v_{y, j}^{(0)}(\boldsymbol{\phi}) \Phi_{0, \mu}^{(N)}(\boldsymbol{\phi})\right\rangle\right| \\
\leq \sqrt{\sum_{j=1}^{N}\left\langle\Phi_{0, \mu}^{(N)}(\boldsymbol{\phi}), v_{x, j}^{(0)}(\boldsymbol{\phi})\left|B_{\mathrm{P}, z}\left(\mathbf{r}_{j}\right)\right|^{2} v_{x, j}^{(0)}(\boldsymbol{\phi}) \Phi_{0, \mu}^{(N)}(\boldsymbol{\phi})\right\rangle} \\
\times \sqrt{\sum_{j=1}^{N}\left\langle\Phi_{0, \mu}^{(N)}(\boldsymbol{\phi}), v_{y, j}^{(0)}(\boldsymbol{\phi})\left|\left(\frac{\partial^{2} B_{\mathrm{P}, z}}{\partial x \partial y}\right)\left(\mathbf{r}_{j}\right)\right|^{2} v_{y, j}^{(0)}(\boldsymbol{\phi}) \Phi_{0, \mu}^{(N)}(\boldsymbol{\phi})\right\rangle} \\
\leq \quad\left\|B_{\mathrm{P}, z}\right\|_{\infty}\left\|\frac{\partial^{2} B_{\mathrm{P}, z}}{\partial x \partial y}\right\|_{\infty} \sqrt{\sum_{j=1}^{N}\left\langle\Phi_{0, \mu}^{(N)}(\boldsymbol{\phi}),\left(v_{x, j}^{(0)}(\boldsymbol{\phi})\right)^{2} \Phi_{0, \mu}^{(N)}(\boldsymbol{\phi})\right\rangle} \\
\times \sqrt{\sum_{j=1}^{N}\left\langle\Phi_{0, \mu}^{(N)}(\boldsymbol{\phi}),\left(v_{y, j}^{(0)}(\boldsymbol{\phi})\right)^{2} \Phi_{0, \mu}^{(N)}(\boldsymbol{\phi})\right\rangle} .
\end{gathered}
$$

This right-hand side is of order $N$ in the same method as in the above. All the rest of the contributions in the right-hand side of (E.43) can be treated in the same way. Consequently $\delta^{\prime}(\phi)$ of (E.10) has been proved to be bounded uniformly in the number $N$ of the electrons.

Consider the special case with $\mathbf{A}_{\mathrm{P}}=0$ which corresponds to the situation of Theorem 7.8. Then all the contributions except for the first sum in (E.31) and for the first sum in (E.32) are vanishing, and a similar result can be obtained for the double commutator $\left[I_{y}(\phi),\left[H_{0}^{(N)}(\phi), I_{y}(\phi)\right]\right]$. As a result, we have the bound,

$$
\delta^{\prime}(\boldsymbol{\phi}) \leq \frac{2 \ell_{B}^{4}}{\left(\hbar \omega_{c}\right)^{2}} \max _{m+n=2}\left\|D^{(m, n)} W\right\|_{\infty}^{2} .
$$

Combining this with (E.6) and (E.9), we obtain the desired result (7.13) with (7.14). 


\section{F Estimate of the ground state energies $E_{0, \mu}^{(N)}(\phi)$}

In this appendix, we show that all of the ground state energies $E_{0, \mu}^{(N)}(\phi)$ are of order of $N$. Let $\Psi_{0}^{(N)}(\phi)$ be a ground state vector of the Landau Hamiltonian,

$$
H_{\mathrm{L}}^{(N)}(\boldsymbol{\phi})=\sum_{j=1}^{N} \frac{1}{2 m_{e}}\left[\left(p_{x, j}-e B y_{j}+\phi_{x}\right)^{2}+\left(p_{y, j}+\phi_{y}\right)^{2}\right],
$$

for the non-interacting $N$ electrons. Then one has

$$
E_{0, \mu}^{(N)}(\phi) \leq\left\langle\Psi_{0}^{(N)}(\phi), H_{0}^{(N)}(\phi) \Psi_{0}^{(N)}(\phi)\right\rangle+\Delta \mathcal{E}(\phi) \quad \text { for } \mu=1,2, \ldots, q,
$$

where $H_{0}^{(N)}(\phi)$ is the Hamiltonian (5.1) of the present system, and $\Delta \mathcal{E}(\phi)$ is given by

$$
\Delta \mathcal{E}(\phi)=\max _{\mu, \mu^{\prime}}\left|E_{0, \mu}^{(N)}(\phi)-E_{0, \mu^{\prime}}^{(N)}(\phi)\right|
$$

Using the eigenvectors $\varphi_{n, k}^{\mathrm{P}}(\boldsymbol{\phi})$ of (6.7) for the single electron Hamiltonian $\mathcal{H}_{0}(\boldsymbol{\phi})$ of (6.1), the ground state expectation value for $H_{0}^{(N)}(\phi)$ can be written as

$$
\begin{aligned}
& \left\langle\Psi_{0}^{(N)}(\boldsymbol{\phi}), H_{0}^{(N)}(\boldsymbol{\phi}) \Psi_{0}^{(N)}(\boldsymbol{\phi})\right\rangle \\
= & \sum_{n, k}\left\langle\varphi_{n, k}^{\mathrm{P}}(\boldsymbol{\phi}), \mathcal{H}(\boldsymbol{\phi}) \varphi_{n, k}^{\mathrm{P}}(\boldsymbol{\phi})\right\rangle+\sum_{1 \leq i<j \leq N}\left\langle\Psi_{0}^{(N)}(\boldsymbol{\phi}), W^{(2)}\left(\mathbf{r}_{i}-\mathbf{r}_{j}\right) \Psi_{0}^{(N)}(\boldsymbol{\phi})\right\rangle \\
\leq & \sum_{n, k}\left[\mathcal{E}_{n, k}+\frac{\sqrt{2} e}{\sqrt{m_{e}}}\left\|\left|\mathbf{A}_{\mathrm{P}}\right|\right\|_{\infty} \sqrt{\mathcal{E}_{n, k}}+\frac{e^{2}}{2 m_{e}}\left(\left\|\left|\mathbf{A}_{\mathrm{P}}\right|\right\|_{\infty}\right)^{2}+\left\|W^{+}\right\|_{\infty}\right] \\
+ & \sum_{1 \leq i<j \leq N}\left\langle\Psi_{0}^{(N)}(\boldsymbol{\phi}), W^{(2)}\left(\mathbf{r}_{i}-\mathbf{r}_{j}\right) \Psi_{0}^{(N)}(\boldsymbol{\phi})\right\rangle,
\end{aligned}
$$

where $\mathcal{E}_{n, k}=(n+1 / 2) \hbar \omega_{c}$, the Hamiltonian $\mathcal{H}(\phi)$ is given by (6.19), and we have used the inequality (6.22). Clearly the first sum in the right-hand side of the inequality is of order $N$, and so it is enough to estimate the ground state expectation value of the electron-electron interaction energy. But this quantity is of order $N$ in the same way as in Appendix G of ref. [32].

\section{References}

[1] Nakano, H.: A Method of Calculation of Electrical Conductivity. Prog. Theor. Phys. 15, 77-79 (1956)

[2] Kubo, R.: Statistical-Mechanical Theory of Irreversible Processes. I. General Theory and Simple Applications to Magnetic and Conduction Problems. J. Phys. Soc. Jpn. 12, 570-586 (1957)

[3] von Klitzing, K., Dorda, G., Pepper, M.: New Method for High Accuracy Determination of the Fine Structure Constant Based on Quantized Hall Resistance. Phys. Rev. Lett. 45, 494-497 (1980) 
[4] Kawaji, S., Wakabayashi, J.: Temperature Dependence of Transverse and Hall Conductivities of Silicon MOS Inversion Layers under Strong Magnetic Fields. In: Physics in High Magnetic Fields. Chikazumi, S., Miura, N. (eds), pp. 284-287, Springer, Berlin, Heidelberg, New York, 1981

[5] Thouless, D. J., Kohmoto, M., Nightingale, M. P., den Nijs, M.: Quantized Hall Conductance in a Two-Dimensional Periodic Potential. Phys. Rev. Lett. 49, 405-408 (1982)

[6] Kohmoto, M.: Topological Invariant and the Quantization of the Hall Conductance. Ann. Phys. 160, 343-354 (1985)

[7] Aizenman, M., Graf, G. M.: Localization Bounds for an Electron Gas. J. Phys. A31, 6783-6806 (1998)

[8] Bellissard, J., Van Elst, A., Schulz-Baldes, H.: The Noncommutative Geometry of the Quantum Hall Effect. J. Math. Phys. 35, 5373-5451 (1994)

[9] Elgart, A., Schlein, B.: Adiabatic Charge Transport and the Kubo Formula for Landau Type Hamiltonians. Comm. Pure Appl. Math. 57, 590-615 (2004)

[10] Dubrovin, B. A., Novikov, S. P.: Ground States in a Periodic Field. Magnetic Bloch Functions and Vector Bundles. Soviet Math. Dokl. 22, 240-244 (1980); Ground States of a Two-Dimensional Electron in a Periodic Magnetic Field. Soviet Phys. JETP 52, $511-516$ (1980);

Novikov, S. P., Magnetic Bloch Function and Vector Bundles. Typical Dispersion Laws and Their Quantum Numbers. Soviet Math. Dokl. 23, 298-303 (1981)

[11] Kunz, H.: The Quantum Hall Effect for Electrons in a Random Potential. Commun. Math. Phys. 112, 121-145 (1987)

[12] Avron, J. E., Seiler, R., Simon, B.: Quantum Hall Effect and the Relative Index for Projections. Phys. Rev. Lett. 65, 2185-2188 (1990); Charge Deficiency, Charge Transport and Comparison of Dimensions. Commun. Math. Phys. 159, 399-422 (1994)

[13] Koma, T.: The Width of the Hall Conductance Plateaus. in preparation

[14] Avron, J. E., Seiler, R., Simon, B.: Homotopy and Quantization in Condensed Matter Physics. Phys. Rev. Lett. 51, 51-53 (1983)

[15] Simon, B.: Holonomy, the Quantum Adiabatic Theorem, and Berry's Phase. Phys. Rev. Lett. 51, 2167-2170 (1983)

[16] Dana, I., Avron, Y., Zak, J.: Quantised Hall Conductance in a Perfect Crystal. J. Phys. C18, L679-L683 (1985)

[17] Ishikawa, K.: Topological Phenomena in Two-Dimensional Electron Systems. In: Proc. 3rd Int. Symp. Foundations of Quantum Mechanics, Tokyo, 1989, pp. 70-79;

Imai, N., Ishikawa, K., Matsuyama, T., Tanaka, I.: Field Theory in a Strong Magnetic 
Field and the Quantum Hall Effect: Integer Hall Effect. Phys. Rev. B42, 10610-10640 (1990)

[18] Niu, Q., Thouless, D. J.: Quantised Adiabatic Charge Transport in the Presence of Substrate Disorder and Many-Body Interaction. J. Phys. A17, 2453-2462 (1984)

[19] Niu, Q., Thouless, D. J., Wu, Y.-S.: Quantized Hall Conductance as a Topological Invariant. Phys. Rev. B31, 3372-3377 (1985)

[20] Avron, J. E., Seiler, R.: Quantization of the Hall Conductance for General Multiparticle Schrödinger Hamiltonians. Phys. Rev. Lett. 54, 259-262 (1985)

[21] Avron, J. E., Seiler, R., Yaffe, L. G.: Adiabatic Theorems and Applications to the Quantum Hall Effect. Commun. Math. Phys. 110, 33-49 (1987)

[22] Tsui, D. C., Störmer, H. L., Gossard, A. C.: Two-Dimensional Magnetotransport in the Extreme Quantum Limit. Phys. Rev. Lett. 48, 1559-1562 (1982)

[23] Störmer, H. L., Chang, A. M., Tsui, D. C., Hwang, J. C. M., Gossard, A. C., Wiegmann, W.: Fractional Quantization of the Hall Effect. Phys. Rev. Lett. 50, 1953-1956 (1983)

[24] Willett, R., Eisenstein, J. P., Störmer, H. L., Tsui, D. C., Gossard, A. C., English, J. H.: Observation of an Even-Denominator Quantum Number in the Fractional Quantum Hall Effect. Phys. Rev. Lett. 59, 1776-1779 (1987)

[25] Tao, R., Wu, Y.-S.: Gauge Invariance and Fractional Quantum Hall Effect. Phys. Rev. B30, 1097-1098 (1984)

[26] Tao, R., Haldane, F. D. M.: Impurity Effect, Degeneracy, and Topological Invariant in the Quantum Hall Effect. Phys. Rev. B33, 3844-3850 (1986)

[27] Avron, J. E., Yaffe, L. G.: Diophantine Equation for the Hall Conductance of Interacting Electrons on a Torus. Phys. Rev. Lett. 56, 2084-2087 (1986)

[28] Yoshioka, D., Halperin, B. I., Lee, P. A.: Ground State of Tow-Dimensional Electrons in Strong Magnetic Fields and $\frac{1}{3}$ Quantized Hall Effect. Phys. Rev. Lett. 50, 12191222 (1983); The Ground State of the 2D Electrons in a Strong Magnetic Field and the Anomalous Quantized Hall Effect. Surf. Sci. 142, 155-162 (1984)

[29] Yoshioka, D.: Ground State of the Two-Dimensional Charged Particles in a Strong Magnetic Field and the Fractional Quantum Hall Effect. Phys. Rev. B29, 6833-6839 (1984)

[30] Su, W. P.: Ground-State Degeneracy and Fractionally Charged Excitations in the Anomalous Quantum Hall Effect. Phys. Rev. B30, 1069-1072 (1984)

[31] Koma, T.: Spectral Gaps of Quantum Hall Systems with Interactions. J. Stat. Phys. 99, 313-381 (2000) 
[32] Koma, T.: Insensitivity of Quantized Hall Conductance to Disorder and Interactions. J. Stat. Phys. 99, 383-459 (2000)

[33] Fröhlich, J., Kerler, T.: Universality in Quantum Hall Systems. Nucl. Phys. B 354, 369-417 (1991);

Fröhlich, J., Zee, A.: Large Scale Physcs of the Quantum Hall Fluid. Nucl. Phys. B 364, 517-540 (1991);

Fröhlich, J., Studer, U. M.: Gauge Invariance in Non-Relativistic Many Body Theory. Int. J. Mod. Phys. B6, 2201-2208 (1992); $U(1) \times S U(2)$-Gauge Invariance of Non-Relativistic Quantum Mechanics, and Generalized Hall Effects, Commun. Math. Phys. 148, 553-600 (1992); Gauge Invariance and Current Algebra in Non-Relativistic Many-Body Theory. Rev. Mod. Phys. 65, 733-802 (1993);

Fröhlich, J., Thiran, E.: Integral Quadratic Forms, Kac-Moody Algebras, and Fractional Quantum Hall Effect. J. Stat. Phys. 76, 209-283 (1994);

Fröhlich, J., Kerler, T., Studer, U. M., Thiran, E.: Structuring the Set of Incompressible Quantum Hall Fluids. Nucl. Phys. B 453, 670-704 (1995);

Fröhlich, J., Studer, U. M., Thiran, E.: A Classification of Quantum Hall Fluid. J. Stat. Phys. 86, 821-897 (1997);

Fröhlich, J., Pedrini, B., Schweigert, C., Walcher, J.: Universality in Quantum Hall Systems: Coset Construction of Incompressible States. J. Stat. Phys. 103, 527-567 (2001)

[34] Connes, A.: Noncommutative Geometry, Academic Press, San Diego, 1994

[35] Xia, J.: Geometric Invariants of the Quantum Hall Effect. Commun. Math. Phys. 119, 29-50 (1988)

[36] Nakano, F.: Calculation of the Hall Conductivity by Adiabatic Approximation. J. Math. Sci. Univ. Tokyo 4, 351-371 (1997); Calculation of the Hall Conductivity by Abel Limit. Ann. Inst. Henri Poincaré, 69, 441-455 (1998)

[37] Chandelier, F., Georgelin, Y., Masson, T., Wallet, J.-C.: Quantum Hall Conductivity in a Landau Type Model with a Realistic Geometry. Ann. Phys. 305, 60-78 (2003)

[38] Niu, Q., Thouless, D. J.: Quantum Hall Effect with Realistic Boundary Conditions Phys. Rev. B35, 2188-2197 (1987)

[39] Atiyah, M. F.: Geometry of Yang-Mills Fields, Lezioni Fermiane, Accad. Naz. Lincei \& Scuola Norm. Sup. Pisa, 1979

[40] Cycon, H. L., Froese, R. G., Kirsch, W., Simon, B.: Schrödinger Operators with Application to Quantum Mechanics and Global Geometry, Springer-Verlag, 1987

[41] Gilkey, P. B.: Invariance Theory, the Heat Equation, and the Atiyah-Singer Index Theorem, 2nd ed. CRC Press, Boca Raton, 1995

[42] Weinberg, S.: The Quantum Theory of Fields, vol. II, Modern Applications, Cambridge University Press, 1996 
[43] Zak, J.: Magnetic Translation Group. Phys. Rev. 134, A1602-A1606 (1964); Magnetic Translation Group. II. Irreducible Representations. ibid. 134, A1607-A1611 (1964)

[44] Greenwood, D. A.: The Boltzmann Equation in the Theory of Electrical Conduction in Metals. Proc. Phys. Soc. 71, 585-596 (1958)

[45] Nakano, F., Kaminaga, M.: Absence of Transport under a Slowly Varying Potential in Disordered Systems. J. Stat. Phys. 97, 917 (1999)

[46] Nakamura, S., Bellissard, J.: Low Energy Bands Do Not Contribute to Quantum Hall Effect. Commun. Math. Phys. 31, 283-305 (1990)

[47] Kato, T.: Perturbation Theory for Linear Operators, 2nd ed. Springer, Berlin, Heidelberg, New York, 1980

[48] Kato, T.: Linear Evolution Equations of "Hyperbolic" Type I. J. Fac. Sci. Univ. Tokyo, Sec. I. A 17, 241-258 (1970); Linear Evolution Equations of "Hyperbolic" Type II. J. Math. Soc. Japan. 25, 648-666 (1973)

[49] Fujikawa, K.: Path Integral for Gauge Theories with Fermions. Phys. Rev. D21, $2848-2858(1980)$

[50] Reed, M., Simon, B.: Methods of Modern Mathematical Physics, vol. IV, Analysis of Operators, Academic Press, New York, 1978

[51] Horsch, P., von der Linden, W.: Spin-Correlations and Low Lying Excited States of the Spin-1/2 Heisenberg Antiferromagnet on a Square Lattice. Z. Phys. B72, 181-193 (1988)

[52] Koma, T., Tasaki, H.: Symmetry Breaking and Finite-Size Effects in Quantum ManyBody Systems. J. Stat. Phys. 76, 745-803 (1994) 